JOURNAL OF THE

AMERICAN MATHEMATICAL SOCIETY

Volume 15, Number 1, Pages 27-75

S 0894-0347(01)00380-0

Article electronically published on July 31, 2001

\title{
COMPACTIFYING THE SPACE OF STABLE MAPS
}

\author{
DAN ABRAMOVICH AND ANGELO VISTOLI
}

\section{Contents}

1. Introduction

2. Generalities on stacks 30

3. Twisted objects 35

4. Twisted curves and twisted stable maps $\quad 41$

5. The category $\mathcal{K}_{g, n}(\mathcal{M}, d)$ is an algebraic stack 47

6. The weak valuative criterion $\quad 54$

7. Boundedness 56

8. Some properties and generalizations $\quad 62$

9. Functoriality of the stack of twisted stable maps $\quad 64$

Appendix A. Grothendieck's existence theorem for tame stacks $\quad 70$

References

\section{INTRODUCTION}

We fix a noetherian base scheme $\mathbb{S}$.

1.1. The problem of moduli of families. Consider a Deligne-Mumford stack $\mathcal{M}$ (definition in Section 2.1) admitting a projective coarse moduli scheme M. Given a curve $C$, it is often natural to consider morphisms $f: C \rightarrow \mathcal{M}$ (or equivalently, objects $f \in \mathcal{M}(C)$ ); in case $\mathcal{M}$ is the moduli stack of geometric objects, these morphisms correspond to families over $C$. For example, if $\mathcal{M}=\overline{\mathcal{M}}_{\gamma}$, the stack of stable curves of genus $\gamma$, then morphisms $f: C \rightarrow \mathcal{M}$ correspond to families of stable curves of genus $\gamma$ over $C$; and if $\mathcal{M}=\mathcal{B} G$, the classifying stack of a finite group $G$, we get principal $G$-bundles over $C$. It is interesting to study moduli of such objects; moreover, it is natural to study such moduli as $C$ varies and to find a natural compactification for such moduli.

One approach is suggested by Kontsevich's moduli of stable maps.

Received by the editors May 11, 2000.

2000 Mathematics Subject Classification. Primary 14H10, 14D20.

The first author's research was partially supported by National Science Foundation grant DMS9700520 and by an Alfred P. Sloan research fellowship.

The second author's research was partially supported by the University of Bologna, funds for selected research topics. 
1.2. Stable maps. First consider a projective scheme $\mathbf{M} \rightarrow \mathbb{S}$ with a fixed ample sheaf $\mathcal{O}_{\mathbf{M}}(1)$. Given integers $g, n, d$, it is known that there exists a proper algebraic stack, which here we denote by $\mathcal{K}_{g, n}(\mathbf{M}, d)$, of stable, $n$-pointed maps of genus $g$ and

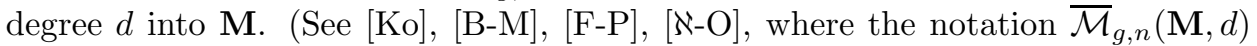
is used. Here we tried to avoid using $\mathcal{M}, \mathbf{M}$ with two meanings.) This stack admits a projective coarse moduli space $\mathbf{K}_{g, n}(\mathbf{M}, d)$. If one avoids "small" residue characteristics in $\mathbb{S}$, which depend on $g, n, d$ and $\mathbf{M}$, then this stack is in fact a proper Deligne-Mumford stack.

1.3. Stable maps into stacks. Now fix a proper Deligne-Mumford stack $\mathcal{M} \rightarrow \mathbb{S}$ admitting a projective coarse moduli space $\mathbf{M} \rightarrow \mathbb{S}$ on which we fix an ample sheaf as above. We further assume that $\mathcal{M}$ is tame, that is, for any geometric point $s: \operatorname{Spec} \Omega \rightarrow \mathcal{M}$, the group $\operatorname{Aut}_{\mathrm{Spec} \Omega}(s)$ has order prime to the characteristic of the algebraically closed field $\Omega$.

It is tempting to mimic Kontsevich's construction as follows: Let $C$ be a nodal projective connected curve; then a morphism $C \rightarrow \mathcal{M}$ is said to be a stable map of degree $d$ if the associated morphism to the coarse moduli scheme $C \rightarrow \mathbf{M}$ is a stable map of degree $d$.

It follows from our results below that the category of stable maps into $\mathcal{M}$ is a Deligne-Mumford stack. A somewhat surprising point is that it is not complete.

To see this, we fix $g=2$ and consider the specific case of $\mathcal{B} G$ with $G=(\mathbb{Z} / 3 \mathbb{Z})^{4}$. Any smooth curve $C$ of genus 2 admits a connected principal $G$-bundle, corresponding to a surjection $H_{1}(C, \mathbb{Z}) \rightarrow G$, thus giving a map $C \rightarrow \mathcal{B} G$. If we let $C$ degenerate to a nodal curve $C_{0}$ of geometric genus 1 , then $H_{1}\left(C_{0}, \mathbb{Z}\right) \simeq \mathbb{Z}^{3}$, and since there is no surjection $\mathbb{Z}^{3} \rightarrow G$, there is no connected principal $G$-bundle over $C_{0}$. This means that there can be no limiting stable map $C_{0} \rightarrow \mathcal{B} G$ as a degeneration of $C \rightarrow \mathcal{B} G$.

1.4. Twisted stable maps. Our main goal here is to correct this deficiency. In order to do so, we will enlarge the category of stable maps into $\mathcal{M}$. The source curve $\mathcal{C}$ of a new stable map $\mathcal{C} \rightarrow \mathcal{M}$ will acquire an orbispace structure at its nodes. Specifically, we endow it with the structure of a Deligne-Mumford stack.

It is not hard to see how these orbispace structures come about. Let $S$ be the spectrum of a discrete valuation ring $R$ of pure characteristic 0 , with quotient field $K$, and let $C_{K} \rightarrow \eta \in S$ be a nodal curve over the generic point, together with a map $C_{K} \rightarrow \mathcal{M}$ of degree $d$, whose associated map $C_{K} \rightarrow \mathbf{M}$ is stable. We can exploit the fact that $\mathcal{K}_{g, 0}(\mathbf{M}, d)$ is complete; after a ramified base change on $S$ the induced map $C_{K} \rightarrow \mathbf{M}$ will extend to a stable map $C \rightarrow \mathbf{M}$ over $S$. Let $C_{\text {sm }}$ be the smooth locus of the morphism $C \rightarrow S$; Abhyankar's lemma, plus a fundamental purity lemma (see Lemma 2.4.1 below) shows that after a suitable base change we can extend the map $C_{K} \rightarrow \mathcal{M}$ to a map $C_{\text {sm }} \rightarrow \mathcal{M}$; in fact the purity lemma fails to apply only at the "new" nodes of the central fiber, namely those which are not in the closure of nodes in the generic fiber. On the other hand, if $p \in C$ is such a node, then on an étale neighborhood $U$ of $p$, the curve $C$ looks like

$$
u v=t^{r},
$$

where $t$ is the parameter on the base. By taking $r$-th roots,

$$
u=u_{1}^{r}, v=v_{1}^{r},
$$


we have a nonsingular cover $V_{0} \rightarrow U$ where $V_{0}$ is defined by $u_{1} v_{1}=t$. The purity lemma applies to $V_{0}$, so the composition $V_{0 K} \rightarrow C_{K} \rightarrow \mathcal{M}$ extends over all of $V_{0}$. There is a minimal intermediate cover $V_{0} \rightarrow V \rightarrow U$ such that the family extends already over $V$; this $V$ will be of the form $x y=t^{r / m}$, and the map $V \rightarrow U$ is given by $u=x^{m}, v=y^{m}$. Furthermore, there is an action of the group $\boldsymbol{\mu}_{m}$ of roots of 1 , under which $\alpha \in \boldsymbol{\mu}_{m}$ sends $x$ to $\alpha x$ and $y$ to $\alpha^{-1} y$, and $V / \boldsymbol{\mu}_{m}=U$. This gives the orbispace structure $\mathcal{C}$ over $C$, and the map $C_{K} \rightarrow \mathcal{M}$ extends to a map $\mathcal{C} \rightarrow \mathcal{M}$.

This gives the flavor of our definition.

We define a category $\mathcal{K}_{g, n}(\mathcal{M}, d)$, fibered over $\mathcal{S} c h / \mathbb{S}$, of twisted stable n-pointed maps $\mathcal{C} \rightarrow \mathcal{M}$ of genus $g$ and degree $d$. This category is given in two equivalent realizations: one as a category of stable twisted $\mathcal{M}$-valued objects over nodal pointed curves endowed with atlases of orbispace charts (see Definition 3.7.2); the other as a category of representable maps from pointed nodal Deligne-Mumford stacks into $\mathcal{M}$, such that the map on coarse moduli spaces is stable (see Definition 4.3.1). In our treatment, both realizations are used in proving our main theorem:

Theorem 1.4.1. (1) The category $\mathcal{K}_{g, n}(\mathcal{M}, d)$ is a proper algebraic stack.

(2) The coarse moduli space $\mathbf{K}_{g, n}(\mathcal{M}, d)$ of $\mathcal{K}_{g, n}(\mathcal{M}, d)$ is projective.

(3) There is a commutative diagram

$$
\begin{array}{cccc}
\mathcal{K}_{g, n}(\mathcal{M}, d) & \rightarrow & \mathcal{K}_{g, n}(\mathbf{M}, d) \\
\downarrow & & \downarrow \\
\mathbf{K}_{g, n}(\mathcal{M}, d) & \rightarrow & \mathbf{K}_{g, n}(\mathbf{M}, d)
\end{array}
$$

where the top arrow is proper, quasifinite, relatively of Deligne-Mumford type and tame, and the bottom arrow is finite. In particular, if $\mathcal{K}_{g, n}(\mathbf{M}, d)$ is a Deligne-Mumford stack, then so is $\mathcal{K}_{g, n}(\mathcal{M}, d)$.

\subsection{Some applications and directions of further work.}

(1) In our paper $\aleph$-V2 we studied the situation where $\mathcal{M}=\overline{\mathcal{M}}_{\gamma}$, which gives a complete moduli for fibered surfaces. Some further results for elliptic surfaces are obtained in G. La Nave's thesis La].

(2) The case where $\mathcal{M}$ is the classifying stack of a finite group $G$ allows one to improve on the spaces of admissible covers, give moduli compactifications of spaces of curves with abelian and nonabelian level structures, and, with a suitable choice of the group $G$, show that there is a smooth, fine moduli space for admissible $G$-covers, which is a finite covering of $\overline{\mathcal{M}}_{g}$. This is the subject of our preprint $[\aleph-\mathrm{C}-\mathrm{V}$ ] with Alessio Corti, with some ideas contributed by Johan de Jong. Some of these applications were indicated in our announcement $\aleph-V 1$. This approach to admissible covers is closely related to the work of Wewers [We.

(3) A similar reasoning applies to curves with $r$-spin structures, e.g. theta characteristics. This is studied in $\aleph-J]$.

(4) The recursive nature of the theorem allows one to construct both minimal models and stable reduction for pluri-fibered varieties. This is related to recent work of Mochizuki [Mo] and deserves further study.

(5) In $[\overline{\mathrm{C}-\mathrm{R}}], \mathrm{W}$. Chen and Y. Ruan introduce Gromov-Witten invariants of an orbifold, using a differential geometric counterpart of our stack of twisted stable maps. In our joint work $\aleph-\mathrm{G}-\mathrm{V}$ ] with Tom Graber we give an algebraic treatment of these Gromov-Witten invariants, and in the special case of 3-pointed genus 0 maps of degree 0 , construct the Chen-Ruan product 
with integer coefficients. See also [F-G] for an algebraic treatment of global quotients.

(6) In this paper we verify that $\mathcal{K}_{g, n}(\mathcal{M}, d)$ is a proper stack by going through the conditions one by one. It may be worthwhile to develop a theory of Grothendieck Quot-stacks and deduce our results from such a theory. It seems likely that some of our methods could be useful for developing such a theory.

While our paper was circulating, we were told in 1999 by Maxim Kontsevich that he had also discovered the stack of twisted stable maps, but had not written down the theory. His motivation was in the direction of Gromov-Witten invariants of stacks.

1.6. Acknowledgments. We would like to thank Kai Behrend, Larry Breen, Barbara Fantechi, Ofer Gabber, Johan de Jong, Maxim Kontsevich, and Rahul Pandharipande, for helpful discussions. We are grateful to Laurent Moret-Bailly for providing us with a preprint of the book [L-MB] before it appeared. The first author thanks the Max Planck Institute für Mathematik in Bonn for a visiting period which helped in putting this paper together.

\section{Generalities on Stacks}

2.1. Criteria for a Deligne-Mumford stack. We refer the reader to $\mathrm{Ar}$ and [L-MB] for a general discussion of algebraic stacks (generalizing [D-M]), and to the appendix in $\mathrm{Vi}$ for an introduction. We spell out the conditions here, as we follow them closely in the paper. We are given a category $\mathcal{X}$ along with a functor $\mathcal{X} \rightarrow \mathcal{S}$ ch $/ \mathbb{S}$. We assume

(1) $\mathcal{X} \rightarrow \mathcal{S} c h / \mathbb{S}$ is fibered in groupoids (see $\mathrm{Ar}$, $\S 1$, (a) and (b), or [-MB, Definition 2.1). This means:

(a) for any morphism of schemes $T \rightarrow T^{\prime}$ and any object $\xi^{\prime} \in \mathcal{X}\left(T^{\prime}\right)$ there is an object $\xi \in \mathcal{X}(T)$ and an arrow $\xi \rightarrow \xi^{\prime}$ over $T \rightarrow T^{\prime}$; and

(b) for any diagram of schemes

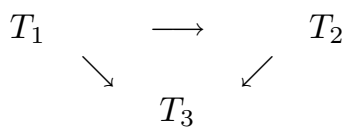

and any objects $\xi_{i} \in \mathcal{X}\left(T_{i}\right)$ sitting in a compatible diagram

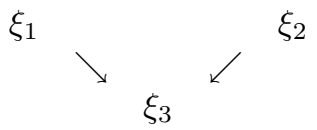

there is a unique arrow $\xi_{1} \rightarrow \xi_{2}$ over $T_{1} \rightarrow T_{2}$ making the diagram commutative.

We remark that this condition is automatic for moduli problems, where $\mathcal{X}$ is a category of families with morphisms given by fiber diagrams.

(2) $\mathcal{X} \rightarrow \mathcal{S} c h / \mathbb{S}$ is a stack, namely:

(a) the Isom functors are sheaves in the étale topology; and

(b) any étale descent datum for objects of $\mathcal{X}$ is effective.

See [Ar], 1.1, or [L-MB], Definition 3.1. 
(3) The stack $\mathcal{X} \rightarrow \mathcal{S}$ ch/S is algebraic, namely:

(a) the Isom functors are representable by separated algebraic spaces of finite type; and

(b) there is a scheme $X$, locally of finite type, and a smooth and surjective morphism $X \rightarrow \mathcal{X}$.

See [-MB, Definition 4.1. Notice that (3a) implies 2a .

These last two conditions are often the most difficult to verify. For the last one, M. Artin has devised a set of criteria for constructing $X \rightarrow \mathcal{X}$ by algebraization of formal deformation spaces (see $\mathrm{Ar}$, Corollary 5.2). Thus, in case $\mathbb{S}$ is of finite type over a field or an excellent Dedekind domain, condition (3b) holds if

(A) $\mathcal{X}$ is limit preserving (see $\mathrm{Ar}, \S 1$ );

(B) $\mathcal{X}$ is compatible with formal completions (see $\mathrm{Ar}, 5.2(3)$ );

(C) Schlessinger's conditions for pro-representability of the deformation functors hold (see [Ar], (2.2) and (2.5)); and

(D) there exists an obstruction theory for $\mathcal{X}$ (see $[\mathrm{Ar},(2.6)$ ) such that

(i) the deformation and obstruction theory is compatible with étale localization ( $\mathrm{Ar}, 4.1(\mathrm{i}))$;

(ii) the deformation theory is compatible with formal completions ( $\mathrm{Ar}$, 4.1 (ii)); and

(iii) the deformation and obstruction theory is constructible ([ $\mathrm{Ar}], 4.1$ (iii)).

Furthermore, we say that $\mathcal{X}$ is a Deligne-Mumford stack if we can choose $X \rightarrow \mathcal{X}$ as in (3b) to be étale. This holds if and only if the diagonal $\mathcal{X} \rightarrow \mathcal{X} \times \mathcal{X}$ is unramified ([L-MB], Théorème 8.1). A morphism $\mathcal{X} \rightarrow \mathcal{X}_{1}$ is of Deligne-Mumford type if for any scheme $Y$ and morphism $Y \rightarrow \mathcal{X}_{1}$ the stack $Y \times_{\mathcal{X}_{1}} \mathcal{X}$ is a Deligne-Mumford stack.

For the notion of properness of an algebraic stack see [L-MB], Chapter 7. Thus a stack $\mathcal{X} \rightarrow \mathbb{S}$ is proper if it is separated, of finite type and universally closed. In [L-MB], Remarque 7.11.2, it is noted that the weak valuative criterion for properness using traits might be insufficient for properness. However, in case $\mathcal{X}$ has finite diagonal, it is shown in [E-H-K-V], Theorem 2.7, that there exists a finite surjective morphism from a scheme $Y \rightarrow \mathcal{X}$. In such a case the usual weak valuative criterion suffices ( $[\mathrm{L}-\mathrm{MB}$, Proposition 7.12).

2.2. Coarse moduli spaces. Recall the following result:

Theorem 2.2.1 (Keel-Mori $[\mathrm{K}-\mathrm{M}]$ ). Let $\mathcal{X}$ be an algebraic stack with finite diagonal over a scheme $S$. There exists an algebraic space $\mathbf{X}$ and a morphism $\mathcal{X} \rightarrow \mathbf{X}$ such that

(1) $\mathcal{X} \rightarrow \mathbf{X}$ is proper and quasifinite;

(2) if $k$ is an algebraically closed field, then $\mathcal{X}(k) /$ Isom $\rightarrow \mathbf{X}(k)$ is a bijection;

(3) whenever $Y \rightarrow S$ is an algebraic space and $\mathcal{X} \rightarrow Y$ is a morphism, then the morphism factors uniquely as $\mathcal{X} \rightarrow \mathbf{X} \rightarrow Y$; more generally

(4) whenever $S^{\prime} \rightarrow S$ is a flat morphism of schemes, and whenever $Y \rightarrow S^{\prime}$ is an algebraic space and $\mathcal{X} \times{ }_{S} S^{\prime} \rightarrow Y$ is a morphism, then the morphism factors uniquely as $\mathcal{X} \times{ }_{S} S^{\prime} \rightarrow \mathbf{X} \times{ }_{S} S^{\prime} \rightarrow Y$; in particular

(5) $\pi_{*} \mathcal{O}_{\mathcal{X}}=\mathcal{O}_{\mathbf{X}}$ 
Recall that an algebraic space $\mathbf{X}$ along with a morphism $\pi: \mathcal{X} \rightarrow \mathbf{X}$ satisfying properties (2) and (3) is called a coarse moduli space (or just moduli space). In particular, the theorem of Keel and Mori shows that coarse moduli spaces of algebraic stacks with finite diagonal exist. Moreover, from (4) and (5) above we have that the formation of a coarse moduli space behaves well under flat base change:

Lemma 2.2.2. Let $\mathcal{X} \rightarrow X$ be a proper quasifinite morphism, where $\mathcal{X}$ is a DeligneMumford stack and $X$ is a noetherian scheme. Let $X^{\prime} \rightarrow X$ be a flat morphism of schemes, and denote $\mathcal{X}^{\prime}=X^{\prime} \times_{X} \mathcal{X}$.

(1) If $X$ is the moduli space of $\mathcal{X}$, then $X^{\prime}$ is the moduli space of $\mathcal{X}^{\prime}$.

(2) If $X^{\prime} \rightarrow X$ is also surjective and $X^{\prime}$ is the moduli space of $\mathcal{X}^{\prime}$, then $X$ is the moduli space of $\mathcal{X}$.

Proof. Given a proper quasifinite morphism $\pi: \mathcal{X} \rightarrow X$, it then exhibits $X$ as a moduli space if and only if $\pi_{*} \mathcal{O}_{\mathcal{X}}=\mathcal{O}_{X}$. If $R \Longrightarrow U$ is an étale presentation of $\mathcal{X}$, and $f: U \rightarrow X$ and $g: R \rightarrow X$ are the induced morphisms, then this condition is equivalent to the exactness of the sequence

$$
0 \longrightarrow \mathcal{O}_{X} \longrightarrow f_{*} \mathcal{O}_{U} \rightrightarrows g_{*} \mathcal{O}_{R}
$$

From this the statement follows.

The prototypical example of a moduli space is given by a group quotient: Let $V$ be a scheme and $\Gamma$ a finite group acting on $V$. Consider the stack $[V / \Gamma]$; see L-MB], 2.4.2. The morphism $[V / \Gamma] \rightarrow V / \Gamma$ exhibits the quotient space $V / \Gamma$ as the moduli space of the stack $[V / \Gamma]$. The following well-known lemma shows that étale-locally, the moduli space of any Deligne-Mumford stack is of this form.

Lemma 2.2.3. Let $\mathcal{X}$ be a separated Deligne-Mumford stack, and $\mathbf{X}$ its coarse moduli space. There is an étale covering $\left\{\mathbf{X}_{\alpha} \rightarrow \mathbf{X}\right\}$, such that for each $\alpha$ there is a scheme $U_{\alpha}$ and a finite group $\Gamma_{\alpha}$ acting on $U_{\alpha}$, with the property that the pullback $\mathcal{X} \times \mathbf{X} \mathbf{X}_{\alpha}$ is isomorphic to the stack-theoretic quotient $\left[U_{\alpha} / \Gamma_{\alpha}\right]$.

Sketch of proof. Let $x_{0}$ be a geometric point of $\mathbf{X}$. Denote by $\mathbf{X}^{\text {sh }}$ the spectrum of the strict henselization of $\mathbf{X}$ at the point $x_{0}$, and let $\mathcal{X}^{\text {sh }}=\mathcal{X} \times{ }_{\mathbf{X}} \mathbf{X}^{\text {sh }}$. If $V \rightarrow \mathcal{X}$ is an étale morphism, with $V$ a scheme, having $x_{0}$ in its image, there is a component $U$ of the pullback $V \times_{\mathbf{x}} \mathbf{X}^{\text {sh }}$ which is finite over $\mathbf{X}^{\text {sh }}$. Denote $R=U \times_{\mathcal{X}^{\text {sh }}} U$. We have that under the first projection $R \rightarrow U$, the scheme $R$ splits as a disjoint union of copies of $U$. Let $\Gamma$ be the set of connected components of $R$, so that $R$ is isomorphic to $U \times \Gamma$. Then the product $R \times{ }_{U} R \rightarrow R$ induces a group structure on $\Gamma$, and the second projection $R \simeq U \times \Gamma \rightarrow U$ defines a group action of $\Gamma$ on $U$, such that $\mathcal{X}^{\text {sh }}$ is the quotient $U / \Gamma$.

We need to descend from $\mathcal{X}^{\text {sh }}$ to get the statement on $\mathcal{X}$. This follows from standard limit arguments.

\subsection{Tame stacks and their coarse moduli spaces.}

Definition 2.3.1. (1) A Deligne-Mumford stack $\mathcal{X}$ is said to be tame if for any geometric point $s: \operatorname{Spec} \Omega \rightarrow \mathcal{X}$, the group $\operatorname{Aut}_{\text {Spec } \Omega}(s)$ has order prime to the characteristic of the algebraically closed field $\Omega$.

(2) A morphism $\mathcal{X} \rightarrow \mathcal{X}_{1}$ of algebraic stacks is said to be tame if for any scheme $Y$ and morphism $Y \rightarrow \mathcal{X}_{1}$ the stack $Y \times_{\mathcal{X}_{1}} \mathcal{X}$ is a tame Deligne-Mumford stack. 
A closely related notion is the following:

Definition 2.3.2. An action of a finite group $\Gamma$ on a scheme $V$ is said to be tame if for any geometric point $s: \operatorname{Spec} \Omega \rightarrow V$, the group $\operatorname{Stab}(s)$ has order prime to the characteristic of $\Omega$.

The reader can verify that a separated Deligne-Mumford stack is tame if and only if the actions of the groups $\Gamma_{\alpha}$ on $V_{\alpha}$ in Lemma 2.2.3 are tame.

In case $\mathcal{X}$ is tame, the formation of coarse moduli spaces commutes with arbitrary morphisms:

Lemma 2.3.3. Let $\mathcal{X}$ be a tame Deligne-Mumford stack, $\mathcal{X} \rightarrow \mathbf{X}$ its moduli space. If $\mathbf{X}^{\prime} \rightarrow \mathbf{X}$ is any morphism of schemes, then $\mathbf{X}^{\prime}$ is the moduli space of the fiber product $\mathbf{X}^{\prime} \times \mathbf{X} \mathcal{X}$. Moreover, if $\mathbf{X}^{\prime}$ is reduced, then it is also the moduli space of $\left(\mathbf{X}^{\prime} \times \mathbf{X} \mathcal{X}\right)_{\text {red }}$.

Proof. By Lemma 2.2.2 this is a local condition in the étale topology of $\mathbf{X}$, so we may assume that $\mathcal{X}$ is a quotient stack of type $[V / \Gamma]$, where $\Gamma$ is a finite group acting on an affine scheme $V=\operatorname{Spec} R$. Moreover, since $\mathcal{X}$ is tame, we may assume that the order of $\Gamma$ is prime to all residue characteristics. Then $\mathbf{X}=\operatorname{Spec} R^{\Gamma}$; if $\mathbf{X}^{\prime}=\operatorname{Spec} S$, then the statement is equivalent to the map $S \rightarrow\left(R \otimes_{R^{\Gamma}} S\right)^{\Gamma}$ being an isomorphism. This (well-known) fact can be shown as follows: Recall that for any $R^{\Gamma}[\Gamma]$-module $M$ the homomorphism

$$
\begin{aligned}
M & \stackrel{\mathrm{Av}_{M}}{\longrightarrow} M^{\Gamma} \\
y & \mapsto \frac{1}{|\Gamma|} \sum_{\gamma \in \Gamma} \gamma \cdot y
\end{aligned}
$$

is a projector exhibiting $M^{\Gamma}$ as a direct summand in $M$. Thus the induced morphism

$$
\operatorname{Av}_{R} \otimes \operatorname{Id}_{S}: R \otimes_{R^{\Gamma}} S \rightarrow R^{\Gamma} \otimes_{R^{\Gamma}} S=S
$$

shows that $S \rightarrow\left(R \otimes_{R^{\Gamma}} S\right)^{\Gamma}$ is injective. The morphism $\operatorname{Av}_{R} \otimes \operatorname{Id}_{S}$ is a lifting of

$$
\operatorname{Av}_{R \otimes_{R^{\Gamma}} S}: R \otimes_{R^{\Gamma}} S \rightarrow\left(R \otimes_{R^{\Gamma}} S\right)^{\Gamma}
$$

which is surjective.

This shows that $\mathbf{X}^{\prime}$ is the moduli space of the fiber product $\mathbf{X}^{\prime} \times \mathbf{X} \mathcal{X}$. The statement about $\left(\mathbf{X}^{\prime} \times \mathbf{X} \mathcal{X}\right)_{\text {red }}$ is immediate. This proves the result.

Let $\mathcal{X}$ be a separated tame stack with coarse moduli scheme $\mathbf{X}$. Consider the projection $\pi: \mathcal{X} \rightarrow \mathbf{X}$. The functor $\pi_{*}$ carries sheaves of $\mathcal{O}_{\mathcal{X}}$-modules to sheaves of $\mathcal{O}_{\mathbf{X}}$-modules.

Lemma 2.3.4. The functor $\pi_{*}$ carries quasicoherent sheaves to quasicoherent sheaves, coherent sheaves to coherent sheaves, and is exact.

Proof. The question is local in the étale topology on $\mathbf{X}$, so we may assume that $\mathcal{X}$ is of the form $[V / \Gamma]$, where $V$ is a scheme and $\Gamma$ a finite group of order prime to all residue characteristics, in particular $\mathbf{X}=V / \Gamma$. Now sheaves on $\mathcal{X}$ correspond to equivariant sheaves on $V$. Denote by $q: V \rightarrow \mathbf{X}$ the projection. If $\mathcal{E}$ is a sheaf on $\mathcal{X}$ corresponding to a $\Gamma$-equivariant sheaf $\tilde{\mathcal{E}}$ on $V$, then $\pi_{*} \mathcal{E}=\left(q_{*} \tilde{\mathcal{E}}\right)^{\Gamma}$, which, by the tameness assumption, is a direct summand in $q_{*} \tilde{\mathcal{E}}$. From this the statement follows. 
2.4. Purity lemma. We recall the following purity lemma from $\aleph-\mathrm{V} 2]$ :

Lemma 2.4.1. Let $\mathcal{M}$ be a separated Deligne-Mumford stack, $\mathcal{M} \rightarrow \mathbf{M}$ the coarse moduli space. Let $X$ be a separated scheme of dimension 2 satisfying Serre's condition $S_{2}$. Let $P \subset X$ be a finite subset consisting of closed points, $U=X \backslash P$. Assume that the local fundamental groups of $U$ around the points of $P$ are trivial.

Let $f: X \rightarrow \mathbf{M}$ be a morphism. Suppose there is a lifting $\tilde{f}_{U}: U \rightarrow \mathcal{M}$ :

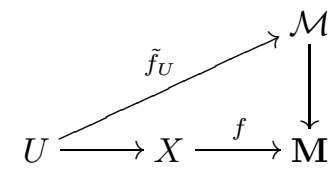

Then the lifting extends to $X$ :

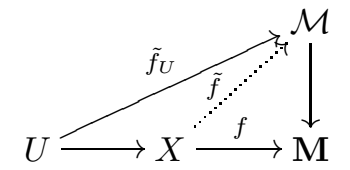

and $\tilde{f}$ is unique up to a unique isomorphism.

Proof. By the descent axiom for $\mathcal{M}$ (see 2.1(2)) the problem is local in the étale topology, so we may replace $X$ and $\mathbf{M}$ with the spectra of their strict henselizations at a geometric point; then we can also assume that we have a universal deformation space $V \rightarrow \mathcal{M}$ which is finite. Now $U$ is the complement of the closed point, $U$ maps to $\mathcal{M}$, and the pullback of $V$ to $U$ is finite and étale, so it has a section, because $U$ is simply connected; consider the corresponding map $U \rightarrow V$. Let $Y$ be the scheme-theoretic closure of the graph of this map in $X \times_{\mathbf{M}} V$. Then $Y \rightarrow X$ is finite and is an isomorphism on $U$. Since $X$ satisfies $S_{2}$, the morphism $Y \rightarrow X$ is an isomorphism.

Remark 2.4.2. The reader can verify that the statement and proof work in higher dimension. See also related lemmas in [Mo].

Corollary 2.4.3. Let $X$ be a smooth surface over a field, $p \in X$ a closed point with complement $U$. Let $X \rightarrow \mathbf{M}$ and $U \rightarrow \mathcal{M}$ be as in the purity lemma. Then there is a lifting $X \rightarrow \mathcal{M}$.

Corollary 2.4.4. Let $X$ be a normal crossings surface over a field $k$, namely a surface which is étale locally isomorphic to $\operatorname{Spec} k[u, v, t] /(u v)$. Let $p \in X$ be a closed point with complement $U$. Let $X \rightarrow \mathbf{M}$ and $U \rightarrow \mathcal{M}$ be as in the purity lemma. Then there is a lifting $X \rightarrow \mathcal{M}$.

Proof. In both cases $X$ satisfies condition $S_{2}$ and the local fundamental group around $p$ is trivial, hence the purity lemma applies.

\subsection{Descent of equivariant objects.}

Lemma 2.5.1. Let $R$ be a local ring with residue field $k$, let $U=\operatorname{Spec} R, u_{0}=$ Spec $k$, let $\mathcal{M}$ be a Deligne-Mumford stack, and let $\eta$ be an object of $\mathcal{M}(U)$. Assume we have a pair of compatible actions of a finite group $\Gamma$ on $R$ and on $\eta$, in such a way that the induced actions of $\Gamma$ on $k$ and on the pullback $\eta_{0}=\left.\eta\right|_{u_{0}}$ are trivial. Then there exists an object $\eta^{\prime}$ of $\mathcal{M}$ on the quotient $U / \Gamma=\operatorname{Spec}\left(R^{\Gamma}\right)$, and a $\Gamma$ invariant lifting $\eta \rightarrow \eta^{\prime}$ of the projection $U \rightarrow U / \Gamma$. Furthermore, if $\eta^{\prime \prime}$ is another 
such object over $U / \Gamma$, there is a unique isomorphism $\eta^{\prime} \simeq \eta^{\prime \prime}$ over the identity of $U / \Gamma$, which is compatible with the two arrows $\eta \rightarrow \eta^{\prime}$ and $\eta \rightarrow \eta^{\prime \prime}$.

As a consequence of the unicity statement, suppose that we have a triple $(\alpha, \beta, \gamma)$, where $\gamma: \Gamma \simeq \Gamma$ is a group isomorphism, and $\alpha: \eta \simeq \eta$ and $\beta: U \simeq U$ are compatible $\gamma$-equivariant isomorphisms. Then the given arrow $\eta \rightarrow \eta^{\prime}$ and its composition with $\alpha$ both satisfy the conditions of the lemma, so there is an induced isomorphism $\bar{\alpha}: \eta^{\prime} \simeq \eta^{\prime}$.

Corollary 2.5.2. Let $R, U, k, u_{0}, \eta, \eta_{0}$ be as in the previous lemma. Let $G$ be a finite group acting compatibly on $R$ and on $\eta$. Let $\Gamma$ be the normal subgroup of $G$ consisting of elements acting on $k$ and $\eta_{0}$ as the identity. Then there exist a $G / \Gamma$-equivariant object $\eta^{\prime}$ on the quotient $U / \Gamma$, and a $G$-equivariant arrow $\eta \rightarrow \eta^{\prime}$ compatible with the projection $U \rightarrow U / \Gamma$.

Proof of the corollary. The action is defined as follows. If $g$ is an element of $G$, call $\alpha: \eta \simeq \eta$ and $\beta: U \simeq U$ the induced arrows, and $\gamma: \Gamma \simeq \Gamma$ the conjugation by $g$. Then the image of $g$ in $G / \Gamma$ acts on $\eta^{\prime}$ via the isomorphism $\bar{\alpha}: \eta^{\prime} \simeq \eta^{\prime \prime}$ defined above. One checks easily that this defines an action with the required properties.

Proof of the lemma. First note that if $R^{\text {sh }}$ is the strict henselization of $R$, the condition on the action of $\Gamma$ allows one to lift it to $R^{\text {sh }}$. Also, the statement that we are trying to prove is local in the étale topology, so by standard limit arguments we can assume that $R$ is strictly henselian. Replacing $\mathbf{M}$ by the spectrum of the strict henselization of its local ring at the image of the closed point of $R$, we can assume that $\mathcal{M}$ is of the form $[V / H]$, where $V$ is a scheme and $H$ is a finite group. Then the object $\eta$ corresponds to a principal $H$-bundle $P \rightarrow U$, on which $\Gamma$ acts compatibly with the action of $\Gamma$ on $U$, and an $H$-equivariant and $\Gamma$-invariant morphism $P \rightarrow V$. Since $U$ is strictly henselian, the bundle $P \rightarrow U$ is trivial, so $P$ is a disjoint union of copies of $\operatorname{Spec} R$, and the group $\Gamma$ permutes these copies; furthermore the hypothesis on the action of $\Gamma$ on the closed fiber over the residue field insures that $\Gamma$ sends each component into itself. The thesis follows easily.

We note that Lemma 2.5.1 can be proven for an arbitrary algebraic stack $\mathcal{M}$ as an application of the notion of relative moduli spaces, which we did not discuss here. Briefly, the assumptions of the lemma give a morphism $[U / \Gamma] \rightarrow \mathcal{M}$. Using Lemma 2.2.2, it can be shown that there is a relative coarse moduli space, namely a representable morphism $\mathcal{V} \rightarrow \mathcal{M}$ such that $\mathcal{V} \times{ }_{\mathcal{M}} Y$ gives the coarse moduli space of $[U / \Gamma] \times{ }_{\mathcal{M}} Y$ for any scheme $Y$ and flat morphism $Y \rightarrow \mathcal{M}$. The fact that $\Gamma$ acts trivially on $\eta_{0}$, together with Lemmas 2.2.2 and 4.4.3 imply that $\mathcal{V}=U / \Gamma$.

\section{TWISTED OBJECTS}

Our goal in this section is to introduce the notion of a stable twisted object. This is a representable $\mathcal{M}$-valued object on a suitable atlas of orbispace charts on a nodal curve. Basic charts have the form $\left[\operatorname{Spec} k[\xi, \eta] / \boldsymbol{\mu}_{r}\right]$ at a node, or $\left[\operatorname{Spec} k[\xi] / \boldsymbol{\mu}_{r}\right]$ along a marking, where $\boldsymbol{\mu}_{r}$ acts freely away from the origin; for completeness of the picture we allow more general charts. The category of stable twisted objects is a concrete, yet somewhat technical, incarnation of our stack $\mathcal{K}_{g, n}(\mathcal{M}, d)$, which is convenient in many steps of our proof of Theorem 1.4.1. 
3.1. Divisorially marked curves. The following definition is a local version of the standard definition of pointed curve; its advantage is that it is stable under localization in the étale topology.

Definition 3.1.1. A divisorially n-marked nodal curve, or simply n-marked curve $\left(U \rightarrow S, \Sigma_{i}\right)$, consists of a nodal curve $\pi: U \rightarrow S$, together with a sequence of $n$ pairwise disjoint closed subschemes $\Sigma_{1}, \ldots, \Sigma_{n} \subset U$ whose supports do not contain any of the singular points of the fibers of $\pi$, and such that the projections $\Sigma_{i} \rightarrow S$ are étale. (Any of the subschemes $\Sigma_{i}$ may be empty.)

If more than one curve is considered, we will often use the notation $\Sigma_{i}^{U}$ to specify the curve $U$. On the other hand, we will often omit the subschemes $\Sigma_{i}^{U}$ from the notation $\left(U \rightarrow S, \Sigma_{i}^{U}\right)$ if there is no risk of confusion.

A nodal $n$-pointed curve $C \rightarrow S$ is considered an $n$-marked curve by taking as the $\Sigma_{i}^{C}$ the images of the sections $S \rightarrow C$.

Definition 3.1.2. If $\left(U \rightarrow S, \Sigma_{i}\right)$ is an $n$-marked nodal curve, we define the special locus of $U$, denoted by $U_{\mathrm{sp}}$, to be the union of the $\Sigma_{i}$ with the singular locus of the projection $U \rightarrow S$, with its natural scheme structure (this makes the projection $U_{\mathrm{sp}} \rightarrow S$ unramified). The complement of $U_{\mathrm{sp}}$ will be called the general locus of $U$, and denoted by $U_{\text {gen }}$.

Definition 3.1.3. If $\left(U \rightarrow S, \Sigma_{i}^{U}\right)$ is a marked curve, and $S^{\prime} \rightarrow S$ is an arbitrary morphism, we define the pullback to be $\left(U^{\prime} \rightarrow S^{\prime}, \Sigma_{i}^{U^{\prime}}\right)$, where $U^{\prime}=S^{\prime} \times{ }_{S} U$ and $\Sigma_{i}^{U^{\prime}}=S^{\prime} \times_{S} \Sigma_{i}^{U}$.

Definition 3.1.4. If $U \rightarrow S$ and $V \rightarrow S$ are $n$-marked curves, a morphism of $n$-marked curves $f: U \rightarrow V$ is a morphism of $S$-schemes which sends each $\Sigma_{i}^{U}$ into $\Sigma_{i}^{V}$.

A morphism of $n$-marked curves $f: U \rightarrow V$ is called strict if the support of $f^{-1}\left(\Sigma_{i}^{V}\right)$ coincides with the support of $\Sigma_{i}^{U}$ for all $i=1, \ldots, n$, and similarly for the singular locus.

We notice that if a morphism of marked curves $U \rightarrow V$ is strict, then there is an induced morphism of curves $U_{\text {gen }} \rightarrow V_{\text {gen }}$. Furthermore, if $f: U \rightarrow V$ is strict and étale, then $f^{-1}\left(\Sigma_{i}^{V}\right)=\Sigma_{i}^{U}$ scheme-theoretically.

Definition 3.1.5. Let $\left(U \rightarrow S, \Sigma_{i}\right)$ be an $n$-marked curve and $\Gamma$ a finite group. An action of $\Gamma$ on $\left(U, \Sigma_{i}\right)$ is an action of $\Gamma$ on $U$ as an $S$-scheme, such that each element on $\Gamma$ acts via an automorphism, in the sense of Definition 3.1.4, of $U$ as a marked curve on $S$.

If $\Gamma$ is a finite group along with a tame action on a marked curve $U \rightarrow S$, then the quotient $U / \Gamma \rightarrow S$ can be given a marked curve structure by defining $\Sigma_{i}^{U / \Gamma}:=\Sigma_{i}^{U} / \Gamma \subseteq U / \Gamma$. The latter inclusion holds because the orders of stabilizers in $\Gamma$ are assumed to be prime to the residue characteristics, so $\Sigma_{i}^{U} / \Gamma$ is indeed a subscheme of $U / \Gamma$.

Given a morphism $f: U \rightarrow V$ of marked curves, and a tame action of a finite group $\Gamma$ on $U$, leaving $f$ invariant, then there is an induced morphism $U / \Gamma \rightarrow V$ of marked curves.

Definition 3.1.6. Let $\left(U \rightarrow S, \Sigma_{i}\right)$ be an $n$-marked curve, with an action of a finite group $\Gamma$, and let $\mathcal{M}$ be a Deligne-Mumford stack. Given $\eta \in \mathcal{M}(U)$, an essential 
action of $\Gamma$ on $(\eta, U)$ is a pair of compatible actions of $\Gamma$ on $\eta$ and on $\left(U \rightarrow S, \Sigma_{i}\right)$, with the property that if $g$ is an element of $\Gamma$ different from the identity and $u_{0}$ is a geometric point of $U$ fixed by $g$, then the automorphism of the pullback of $\eta$ to $u_{0}$ induced by $g$ is not trivial.

\subsection{Generic objects and charts.}

Definition 3.2.1. Let $C \rightarrow S$ be an $n$-pointed nodal curve. A generic object on $C$ is an object of $\mathcal{M}\left(C_{\text {gen }}\right)$.

We will often write $(\xi, C)$ for a generic object $\xi$ on a curve $C$.

Definition 3.2.2. Let $C \rightarrow S$ be an $n$-pointed nodal curve and $\xi$ a generic object on $C$. A chart $(U, \eta, \Gamma)$ for $\xi$ consists of the following collection of data.

(1) An $n$-marked curve $U \rightarrow S$ and a strict morphism $\phi: U \rightarrow C$.

(2) An object $\eta$ of $\mathcal{M}(U)$.

(3) An arrow $\left.\eta\right|_{U_{\text {gen }}} \rightarrow \xi$ in $\mathcal{M}$ compatible with the restriction $\left.\phi\right|_{U_{\text {gen }}}: U_{\text {gen }} \rightarrow$ $C_{\text {gen }}$.

(4) A finite group $\Gamma$.

(5) A tame, essential action of $\Gamma$ on $(\eta, U)$.

Furthermore, we require that the following conditions be satisfied.

a: The actions of $\Gamma$ leave the morphism $U \rightarrow C$ and the arrow $\left.\eta\right|_{U_{\text {gen }}} \rightarrow \xi$ invariant.

b: The induced morphism $U / \Gamma \rightarrow C$ is étale.

The following gives a local description of the action of $\Gamma$.

Proposition 3.2.3. Let $(U, \eta, \Gamma)$ be a chart for a generic object $\xi$ on a pointed nodal curve $C \rightarrow S$. Then the action of $\Gamma$ on $\phi^{-1} C_{\text {gen }}$ is free.

Furthermore, if $s_{0}$ is a geometric point of $S$ and $u_{0}$ a nodal point of the fiber $U_{s_{0}}$ of $U$ over $s_{0}$, then

(1) the stabilizer $\Gamma^{\prime}$ of $u_{0}$ is a cyclic group which sends each of the branches of $U_{s_{0}}$ to itself;

(2) if $k$ is the order of $\Gamma^{\prime}$, then a generator of $\Gamma^{\prime}$ acts on the tangent space of each branch by multiplication with a primitive $k$-th root of 1 .

In particular, each nodal point of $U_{s_{0}}$ is sent to a nodal point of $C_{s_{0}}$.

Proof. The first statement follows from the definition of an essential action and the invariance of the arrow $\left.\eta\right|_{U_{\text {gen }}} \rightarrow E$.

As for (1), observe that if the stabilizer $\Gamma^{\prime}$ of $u_{0}$ did not preserve the branches of $U_{s_{0}}$, then the quotient $U_{s_{0}} / \Gamma^{\prime}$, which is étale at the point $u_{0}$ over the fiber $C_{s_{0}}$, would be smooth over $S$ at $u_{0}$, so $u_{0}$ would be in the inverse image of $C_{\text {gen }}$. From the first part of the proposition it would follow that $\Gamma^{\prime}$ is trivial, a contradiction.

So $\Gamma^{\prime}$ acts on each of the two branches individually. The action on each branch must be faithful because it is free on the complement of the set of nodes; this means that the representation of $\Gamma^{\prime}$ in each of the tangent spaces to the branches is faithful, and this implies the final statement.

Definition 3.2.4. A chart is called balanced if for any nodal point of any geometric fiber of $U$, the two roots of 1 describing the action of a generator of the stabilizer on the tangent spaces to each branch of $U$ are inverse to each other. 
3.3. The transition scheme. Let $\xi$ be a generic object over a nodal curve $C \rightarrow S$, and $\left(U_{1}, \eta_{1}, \Gamma_{1}\right),\left(U_{2}, \eta_{2}, \Gamma_{2}\right)$ two charts; call $\operatorname{pr}_{i}: U_{1} \times{ }_{C} U_{2} \rightarrow U_{i}$ the $i$-th projection. Consider the scheme

$$
I=\underset{U_{1} \times{ }_{C} U_{2}}{\operatorname{Isom}}\left(\operatorname{pr}_{1}^{*} \eta_{1}, \operatorname{pr}_{2}^{*} \eta_{2}\right)
$$

over $U_{1} \times_{C} U_{2}$ representing the functor of isomorphisms of the two objects $\operatorname{pr}_{1}^{*} \eta_{1}$ and $\operatorname{pr}_{2}^{*} \eta_{2}$.

There is a section of $I$ over the inverse image $\widetilde{U}$ of $C_{\text {gen }}$ in $U_{1} \times_{C} U_{2}$ which corresponds to the isomorphism $\left.\left.\operatorname{pr}_{1}^{*} \eta_{1}\right|_{\widetilde{U}} \simeq \operatorname{pr}_{2}^{*} \eta_{2}\right|_{\widetilde{U}}$ coming from the fact that both $\operatorname{pr}_{1}^{*} \eta_{1}$ and $\operatorname{pr}_{2}^{*} \eta_{2}$ are pullbacks to $\widetilde{U}$ of $\xi$. We will call the scheme-theoretic closure $R$ of this section in $I$ the transition scheme from $\left(U_{1}, \eta_{1}, \Gamma_{1}\right)$ to $\left(U_{2}, \eta_{2}, \Gamma_{2}\right)$; it comes equipped with two projections $R \rightarrow U_{1}$ and $R \rightarrow U_{2}$.

There is also an action of $\Gamma_{1} \times \Gamma_{2}$ on $I$, defined as follows. Let $\left(\gamma_{1}, \gamma_{2}\right) \in \Gamma_{1} \times \Gamma_{2}$, and let $\phi: \operatorname{pr}_{1}^{*} \eta_{1} \simeq \operatorname{pr}_{2}^{*} \eta_{2}$ be an isomorphism over $U_{1} \times_{C} U_{2}$; then define $\left(\gamma_{1}, \gamma_{2}\right) \cdot \phi=$ $\gamma_{2} \circ \phi \circ \gamma_{1}^{-1}$. This action of $\Gamma_{1} \times \Gamma_{2}$ on $I$ is compatible with the action of $\Gamma_{1} \times \Gamma_{2}$ on $U_{1} \times{ }_{C} U_{2}$, and leaves $R$ invariant. It follows from the definition of an essential action that the action of $\Gamma_{1}=\Gamma_{1} \times\{1\}$ and $\Gamma_{2}=\{1\} \times \Gamma_{2}$ on $I$ is free.

\subsection{Compatibility of charts.}

Definition 3.4.1. Two charts $\left(U_{1}, \eta_{1}, \Gamma_{1}\right)$ and $\left(U_{2}, \eta_{2}, \Gamma_{2}\right)$ are compatible if their transition scheme $R$ is étale over $U_{1}$ and $U_{2}$.

Let us analyze this definition. First of all, $R$ is obviously étale over $\left(U_{1}\right)_{\text {gen }}$ and $\left(U_{2}\right)_{\text {gen }}$. Also, since the maps $U_{j} \rightarrow C$ are strict, it is clear that the inverse image of $\Sigma_{i}^{U_{1}}$ in $R$ is set-theoretically equal to the inverse image of $\Sigma_{i}^{U_{2}}$. If the two charts are compatible, this also holds scheme-theoretically.

Now, start from two charts $\left(U_{1}, \eta_{1}, \Gamma_{1}\right)$ and $\left(U_{2}, \eta_{2}, \Gamma_{2}\right)$. Fix two geometric points

$$
u_{1}: \operatorname{Spec} \Omega \rightarrow U_{1} \quad \text { and } \quad u_{2}: \operatorname{Spec} \Omega \rightarrow U_{2}
$$

mapping to the same geometric point $u_{0}: \operatorname{Spec} \Omega \rightarrow C$, and call $\Gamma_{j}^{\prime} \subset \Gamma_{j}$ the stabilizer of $u_{j}$. Also call $U_{1}^{\text {sh }}, U_{2}^{\text {sh }}$ and $C^{\text {sh }}$ the spectra of the strict henselizations of $U_{1}, U_{2}$ and $C$ at the points $u_{1}, u_{2}$ and $u_{0}$ respectively. The action of $\Gamma_{j}$ on $U_{j}$ induces an action of $\Gamma_{j}^{\prime}$ on $U_{j}^{\text {sh }}$. Also call $\eta_{j}^{\text {sh }}$ the pullback of $\eta_{j}$ to $U_{j}^{\text {sh }}$; there is an action of $\Gamma_{j}^{\prime}$ on $\eta_{j}^{\text {sh }}$ compatible with the action of $\Gamma_{j}^{\prime}$ on $U_{j}$. The following essentially says that two charts are compatible if and only if for any choice of $u_{1}$ and $u_{2}$ the two charts are locally isomorphic in the étale topology.

Proposition 3.4.2. The two charts are compatible if and only if for any pair of geometric points $u_{1}$ and $u_{2}$ as above there exist an isomorphism of groups $\theta: \Gamma_{1}^{\prime} \simeq$ $\Gamma_{2}^{\prime}$, a $\theta$-equivariant isomorphism $\phi: U_{1}^{\text {sh }} \simeq U_{2}^{\text {sh }}$ of schemes over $C^{\text {sh }}$, and a compatible $\theta$-equivariant isomorphism $\psi: \eta_{1}^{\mathrm{sh}} \rightarrow \eta_{2}^{\mathrm{sh}}$.

Proof. Consider the spectrum $\left(U_{1} \times_{C} U_{2}\right)^{\text {sh }}$ of the strict henselization of $U_{1} \times_{C} U_{2}$ at the point $\left(u_{1}, u_{2}\right): \operatorname{Spec} \Omega \rightarrow U_{1} \times_{C} U_{2}$, and call $R^{\text {sh }}$ the pullback of $R$ to $\left(U_{1} \times_{C} U_{2}\right)^{\text {sh }}$. Assume that the two charts are compatible. The action of $\Gamma_{1} \times \Gamma_{2}$ on $I$ described above induces an action of $\Gamma_{1}^{\prime} \times \Gamma_{2}^{\prime}$ on $R^{\text {sh }}$, compatible with the action of $\Gamma_{1}^{\prime} \times \Gamma_{2}^{\prime}$ on $\left(U_{1} \times_{C} U_{2}\right)^{\mathrm{sh}}$. The action of $\Gamma_{1}^{\prime}=\Gamma_{1}^{\prime} \times\{1\}$ on the inverse image of $C_{\text {gen }}$ in $R^{\text {sh }}$ is free, and its quotient is the inverse image of $C_{\text {gen }}$ in $U_{2}^{\text {sh }}$; but $R^{\text {sh }}$ is finite and étale over $U_{2}^{\text {sh }}$, so the action of $\Gamma_{1}^{\prime}$ on all of $R^{\text {sh }}$ is free, and $R^{\text {sh }} / \Gamma_{1}^{\prime}=U_{2}$. Analogously the action of $\Gamma_{2}^{\prime}$ on $R^{\text {sh }}$ is free, and $R^{\text {sh }} / \Gamma_{2}^{\prime}=U_{1}$. 
Now, each of the connected components of $R^{\text {sh }}$ maps isomorphically onto both $U_{1}^{\text {sh }}$ and $U_{2}^{\text {sh }}$, because $U_{j}^{\text {sh }}$ is the spectrum of a strictly henselian ring and the projection $R^{\text {sh }} \rightarrow U_{j}^{\text {sh }}$ is étale; this implies in particular that the order of $\Gamma_{1}$ is the same as the number $k$ of connected components, and likewise for $\Gamma_{2}$. Fix one of these components, call it $R_{0}^{\text {sh }}$; then we get isomorphisms $R_{0}^{\text {sh }} \simeq U_{j}^{\text {sh }}$, which yield an isomorphism $\phi: U_{1}^{\text {sh }} \simeq U_{2}^{\text {sh }}$.

Call $\Gamma^{\prime}$ the stabilizer of the component $R_{0}^{\text {sh }}$ inside $\Gamma_{1}^{\prime} \times \Gamma_{2}^{\prime}$; the order of $\Gamma^{\prime}$ is at least $\left|\Gamma_{1}^{\prime} \times \Gamma_{2}^{\prime}\right| / k=k^{2} / k=k$. But the action of $\Gamma_{2}^{\prime}$ on $R^{\text {sh }}$ is free, and so $\Gamma^{\prime} \cap \Gamma_{2}=\{1\}$; this implies that the order of $\Gamma^{\prime}$ is $k$, and the projection $\Gamma^{\prime} \rightarrow \Gamma_{1}^{\prime}$ is an isomorphism. Likewise the projection $\Gamma^{\prime} \rightarrow \Gamma_{2}^{\prime}$ is an isomorphism, so from these we get an isomorphism $\theta: \Gamma_{1}^{\prime} \rightarrow \Gamma_{2}^{\prime}$, and it is easy to check that the isomorphism of schemes $\phi: U_{1}^{\text {sh }} \simeq U_{2}^{\text {sh }}$ is $\theta$-equivariant.

There is also an isomorphism of the pullbacks of $\eta_{1}^{\mathrm{sh}}$ and $\eta_{2}^{\mathrm{sh}}$ to $R_{0}^{\mathrm{sh}}$, coming from the natural morphism $R_{0}^{\text {sh }} \rightarrow I$, which induces an isomorphism $\psi: \eta_{1}^{\text {sh }} \rightarrow \eta_{2}^{\text {sh }}$. This isomorphism is compatible with $\phi$, and it is also $\theta$-equivariant.

Let us prove the converse. Suppose that there exist $\theta, \phi$ and $\psi$ as above. Then there is a morphism $\sigma: U_{1}^{\mathrm{sh}} \times \Gamma_{1}^{\prime} \rightarrow I$ which sends a point $\left(u_{1}, \gamma_{1}\right)$ of $U_{1}^{\mathrm{sh}} \times \Gamma_{1}^{\prime}$ into the point of $I$ lying over the point $\left(u_{1}, \phi \gamma_{1} u_{1}\right)=\left(u_{1}, \theta\left(\gamma_{1}\right) \phi u_{1}\right)$ corresponding to the isomorphism $\gamma_{1} \psi$ of the pullback of $\eta_{1}$ to $u_{1}$ with the pullback of $\eta_{2}$ to $\phi \gamma_{1} u_{1}$. The morphism $\sigma$ is an isomorphism of $U_{1}^{\text {sh }} \times \Gamma_{1}^{\prime}$ with $R^{\text {sh }}$ in the inverse image of $C_{\text {gen }}$; also, from the fact that the action of $\Gamma^{\prime}$ on $\left(\eta_{1}, U_{1}\right)$ is essential, it follows that $\sigma$ is injective. Since the inverse image of $C_{\text {gen }}$ is scheme-theoretically dense in $R^{\text {sh }}$ and $U_{1}^{\text {sh }} \times \Gamma_{1}$ is unramified over $U_{1}$ we see that $\sigma$ is an isomorphism of $U_{1}^{\text {sh }} \times \Gamma_{1}^{\prime}$ with $R^{\text {sh }}$. It follows that $R^{\text {sh }}$ is étale over $U_{1}^{\text {sh }}$; analogously it is étale over $U_{2}^{\text {sh }}$. So $R$ is étale over $U_{1}$ and $U_{2}$ at the points $u_{1}$ and $u_{2}$; since this holds for all $u_{1}$ and $u_{2}$ mapping to the same point of $C$ the conclusion follows.

Compatibility of charts is stable under base change:

Proposition 3.4.3. (1) Let $\left(U_{1}, \eta_{1}, \Gamma_{1}\right),\left(U_{2}, \eta_{2}, \Gamma_{2}\right)$ be two compatible charts for a generic object $\xi$ on $C \rightarrow S$. If $S^{\prime} \rightarrow S$ is an arbitrary morphism, then

$$
\left(S^{\prime} \times{ }_{S} U_{1}, \eta_{1}^{\prime}, \Gamma_{1}\right)
$$

and

$$
\left(S^{\prime} \times{ }_{S} U_{2}, \eta_{2}^{\prime}, \Gamma_{2}\right),
$$

where $\eta_{1}^{\prime}$ and $\eta_{2}^{\prime}$ are the pullbacks of $\eta_{1}$ and $\eta_{2}$ to $S^{\prime} \times{ }_{S} U_{1}$ and $S^{\prime} \times_{S} U_{2}$, are compatible charts for the pullback of $\xi$ to $\left(S^{\prime} \times{ }_{S} C \rightarrow S^{\prime}\right)_{\text {gen }}$.

(2) If $S^{\prime} \rightarrow S$ is étale and surjective, then the converse holds.

The proof is immediate from Proposition 3.4 .2

3.5. The product chart. Given two compatible charts $\left(U_{1}, \eta_{1}, \Gamma_{1}\right),\left(U_{2}, \eta_{2}, \Gamma_{2}\right)$, set $\eta=\operatorname{pr}_{1}^{*} \eta_{1}$ in $\mathcal{M}(R)$. There is an action of $\Gamma$, coming from pulling back the action of $\Gamma_{1}$ on $\eta_{1}$; also the tautological isomorphism $\alpha$ : $\operatorname{pr}_{1}^{*} \eta_{1} \simeq \eta_{2}$ induces an action of $\Gamma_{2}$ on $\eta$. These two actions commute, and therefore define an action of $\Gamma_{1} \times \Gamma_{2}$ on $\eta$, compatible with the action of $\Gamma_{1} \times \Gamma_{2}$ on $\rho$. Also, $R$ has a structure of an $n$-marked curve, by defining $\Sigma_{i}^{R}$ to be the inverse image of $\Sigma_{i}^{U_{1}}$, and the map $R \rightarrow C$ is strict. Then

$$
\left(R, \eta, \Gamma_{1} \times \Gamma_{2}\right)
$$

is a chart, called the product chart. It is compatible with both of the original charts. 


\subsection{Atlases and twisted objects.}

Definition 3.6.1. Fix two nonnegative integers $g$ and $n$. An n-pointed twisted object $(\xi, C \rightarrow S, \mathcal{A})$ of genus $g$ consists of

(1) a proper, $n$-pointed curve $C \rightarrow S$ of finite presentation, with geometrically connected fibers of genus $g$;

(2) a generic object $\xi$ over $C \rightarrow S$; and

(3) a collection $\mathcal{A}=\left\{\left(U_{\alpha}, \eta_{\alpha}, \Gamma_{\alpha}\right)\right\}$ of mutually compatible charts, such that the images of the $U_{\alpha}$ cover $C$.

A collection of charts $\mathcal{A}$ as in (3) is called an atlas.

A twisted object is called balanced if each chart in its atlas is balanced (Definition 3.2.4).

Lemma 3.6.2. If two charts for a twisted object are compatible with all the charts in an atlas, they are mutually compatible.

Furthermore, if the twisted object is balanced, then any chart which is compatible with every chart of the atlas is balanced.

Proof. Both statements are immediate from the local characterization of compatibility in Proposition 3.4.2.

Remark 3.6.3. The lemma above allows one to define a twisted object using a maximal atlas, if one prefers.

Definition 3.6.4. A morphism of twisted objects $(\xi, C \rightarrow S, \mathcal{A})$ to $\left(\xi^{\prime}, C^{\prime} \rightarrow S^{\prime}, \mathcal{A}^{\prime}\right)$ consists of a cartesian diagram

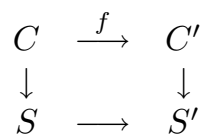

and an arrow $\xi \rightarrow \xi^{\prime}$ lying over the restriction $\left.f\right|_{C_{\text {gen }}}: C_{\text {gen }} \rightarrow C_{\text {gen }}^{\prime}$, with the property that the pullback of the charts in $\mathcal{A}^{\prime}$ are all compatible with the charts in $\mathcal{A}$.

The composition of morphisms of twisted objects is defined to be the one induced by composition of morphisms of curves.

Let $(\xi, C \rightarrow S, \mathcal{A})$ be a twisted object, and $T \rightarrow S$ a morphism. Then, using Proposition 3.4 .3 one can define the pullback of $(\xi, C \rightarrow S, \mathcal{A})$ to $T$ in the obvious way.

\subsection{Stability.}

Lemma 3.7.1. Let $(\xi, C \rightarrow S, \mathcal{A})$ be a twisted object. Then the morphism $C_{\text {gen }} \rightarrow$ $\mathbf{M}$ induced by $\xi$ extends uniquely to a morphism $C \rightarrow \mathbf{M}$.

Proof. The unicity is clear from the fact that $\mathbf{M}$ is separated and $C_{\text {gen }}$ is schemetheoretically dense in $C$. To prove the existence of an extension is a local question in the étale topology; but if $\mathcal{A}=\left\{\left(U_{\alpha}, \eta_{\alpha}, \Gamma_{\alpha}\right)\right\}$, then the objects $\eta_{\alpha}$ induce morphisms $U_{\alpha} \rightarrow \mathbf{M}$, which are $\Gamma_{\alpha}$-equivariant, yielding morphisms $U_{\alpha} / \Gamma_{\alpha} \rightarrow \mathbf{M}$. These morphisms are extensions of the pullback to $\left(U_{\alpha}\right)_{\text {gen }} / \Gamma_{\alpha}$ of the morphism $C_{\text {gen }} \rightarrow$ M. Therefore they descend to $C$. 
We can now define the main object of this section:

Definition 3.7.2. A twisted object is stable if the associated map $C \rightarrow \mathbf{M}$ is Kontsevich stable.

3.8. The stack of stable twisted objects. Fix an ample line bundle $\mathcal{O}_{\mathbf{M}}(1)$ over M. We define a category $\mathcal{K}_{g, n}(\mathcal{M}, d)=\mathcal{K}_{g, n}(\mathcal{M} / \mathbb{S}, d)$ as follows. The objects are stable twisted objects $(\xi, C \rightarrow S, \mathcal{A})$, where $C \rightarrow S$ is a nodal $n$-pointed curve of genus $g$, such that for the associated morphism $f: C \rightarrow \mathbf{M}$ the degree of the line bundle $f^{*} \mathcal{O}_{\mathbf{M}}(1)$ is $d$. The arrows are morphisms of twisted objects.

As stated in Theorem 1.4.1, this category is a proper algebraic stack which is relatively of Deligne-Mumford type over $\mathcal{K}_{g, n}(\mathbf{M}, d)$, admitting a projective coarse moduli space $\mathbf{K}_{g, n}(\mathcal{M}, d)$. The proof of the theorem will begin in Section 5 .

We shall also consider the full subcategory $\mathcal{K}_{g, n}^{\mathrm{bal}}(\mathcal{M}, d)$ of balanced twisted objects. It will be shown in Proposition 8.1.1 that this is an open and closed substack in $\mathcal{K}_{g, n}(\mathcal{M}, d)$, whose moduli space is open and closed in $\mathbf{K}_{g, n}(\mathcal{M}, d)$.

\section{Twisted CURves And twisted Stable maps}

In this section we give a stack-theoretic description of the category $\mathcal{K}_{g, n}(\mathcal{M}, d)$ in terms of twisted stable maps. The language of stacks allows one to circumvent many of the technical details involved in twisted objects, and gives a convenient way of thinking about the category $\mathcal{K}_{g, n}(\mathcal{M}, d)$. It is also convenient for studying deformation and obstruction theory for $\mathcal{K}_{g, n}(\mathcal{M}, d)$.

4.1. Nodal stacks. Let $S$ be a scheme over $\mathbb{S}$. Consider a proper, flat, tame Deligne-Mumford stack $\mathcal{C} \rightarrow S$ of finite presentation, such that its fibers are purely one-dimensional and geometrically connected, with at most nodal singularities. Call $C$ the moduli space of $\mathcal{C}$; by $[\mathrm{K}-\mathrm{M}]$ this exists as an algebraic space.

Proposition 4.1.1. The morphism $C \rightarrow S$ is a proper flat nodal curve of finite presentation, with geometrically connected fibers.

Proof. First of all let us show that $C$ is flat over $S$. We may assume that $S$ is affine; let $R$ be its coordinate ring. Fix a geometric point $c_{0} \rightarrow C$, and call $C^{\text {sh }}$ the strict henselization of $C$ at $c_{0}$. Let $U$ be an étale cover of $\mathcal{C}$, and $u_{0}$ a geometric point of $U$ lying over $c_{0}$; denote by $U^{\text {sh }}$ the strict henselization of $U$ at $u_{0}$. If $\Gamma$ is the automorphism group of the object of $\mathcal{C}$ corresponding to $u_{0}$, then $\Gamma$ acts on $U^{\text {sh }}$, and $C^{\text {sh }}$ is the quotient $U^{\text {sh }} / \Gamma$. Since $\mathcal{C}$ is tame, the order of $\Gamma$ is prime to the residue characteristic of $u_{0}$, therefore the coordinate ring of $C^{\text {sh }}$ is a direct summand, as an $R$-module, of the coordinate ring of $U^{\text {sh }}$, so it is flat over $R$.

The fact that the fibers are nodal follows from the fact that, over an algebraically closed field, the quotient of a nodal curve by a group action is again a nodal curve. Properness is clear; the fact that the morphism $\mathcal{C} \rightarrow C$ is surjective implies that the fibers are geometrically connected. The fact that $C$ is of finite presentation is an easy consequence of the fact that $\mathcal{C}$ is of finite presentation.

Following tradition, when we speak of a "family over $S$ " or "curve over $S$ ", it is always assumed to be of finite presentation. 
Definition 4.1.2. A twisted nodal n-pointed curve over $S$ is a diagram

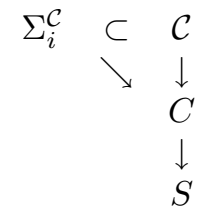

where

(1) $\mathcal{C}$ is a tame Deligne-Mumford stack, proper and of finite presentation over $S$, and étale locally is a nodal curve over $S$;

(2) $\Sigma_{i}^{\mathcal{C}} \subset \mathcal{C}$ are disjoint closed substacks in the smooth locus of $\mathcal{C} \rightarrow S$;

(3) $\Sigma_{i}^{\mathcal{C}} \rightarrow S$ are étale gerbes;

(4) the morphism $\mathcal{C} \rightarrow C$ exhibits $C$ as the coarse moduli scheme of $\mathcal{C}$; and

(5) $\mathcal{C} \rightarrow C$ is an isomorphism over $C_{\text {gen }}$.

Note that if we let $\Sigma_{i}^{C}$ be the coarse moduli spaces of $\Sigma_{i}^{\mathcal{C}}$, then, since $\mathcal{C}$ is tame, the schemes $\Sigma_{i}^{C}$ embed in $C$ (they are the images of $\Sigma_{i}^{\mathcal{C}}$ ), and $C$ becomes a usual nodal pointed curve. We say that $\mathcal{C} \rightarrow S$ is a twisted pointed curve of genus $g$ if $C \rightarrow S$ is a pointed curve of genus $g$.

\subsection{Morphisms of twisted $n$-pointed nodal curves.}

Definition 4.2.1. Let $\mathcal{C} \rightarrow S$ and $\mathcal{C}^{\prime} \rightarrow S^{\prime}$ be twisted $n$-pointed nodal curves. A 1 -morphism (or just a morphism) $F: \mathcal{C} \rightarrow \mathcal{C}^{\prime}$ is a cartesian diagram

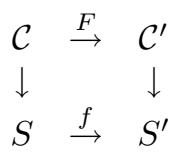

such that $F^{-1} \Sigma_{i}^{\mathcal{C}^{\prime}}=\Sigma_{i}^{\mathcal{C}}$.

If $F, F_{1}: \mathcal{C} \rightarrow \mathcal{C}^{\prime}$ are morphisms, then we define a 2-morphism $F \rightarrow F_{1}$ to be a base-preserving natural transformation. (This implies that it is an isomorphism.)

In this way, twisted pointed curves form a 2-category. However, we have the following:

Proposition 4.2.2. The 2-category of twisted pointed curves is equivalent (in the lax sense, [K-S] ) to a category.

We call the resulting category the category of twisted pointed curves. A morphism in this category is an isomorphism class of 1-morphisms in the 2-category of twisted pointed curves.

Proof. Since all 2-morphisms are invertible, this claim is the same as saying that a 1-arrow in the 2-category cannot have nontrivial automorphisms. The point here is that the stack $\mathcal{C}$ has a dense open representable substack, which is sufficient by the following lemma.

Lemma 4.2.3. Let $F: \mathcal{X} \rightarrow \mathcal{Y}$ be a representable morphism of Deligne-Mumford stacks over a scheme $S$. Assume that there exists a dense open representable substack (i.e. an algebraic space) $U \subseteq \mathcal{X}$ and an open representable substack $V \subseteq \mathcal{Y}$ such that $F$ maps $U$ into $V$. Then any automorphism of $F$ is trivial. 
Proof. (1) First note that the lemma holds if $\mathcal{X}=X$ is an algebraic space: denote by $\eta$ the object of $\mathcal{Y}$ over $X$ corresponding to $F$. The fact that the diagonal $\mathcal{Y} \rightarrow \mathcal{Y} \times{ }_{S} \mathcal{Y}$ of the algebraic stack $\mathcal{Y}$ is separated implies that the isomorphism scheme $\operatorname{Isom}_{X}(\eta, \eta) \rightarrow X$ is separated. Since $V$ is representable, we have that $\operatorname{Isom}_{U}\left(\left.\eta\right|_{U},\left.\eta\right|_{U}\right) \rightarrow U$ is an isomorphism. Thus the unique section over the given open set $U$ has at most one extension to $X$, which gives the assertion in this case.

We will now reduce the general case to this one by descent. We start with some observations.

(2) Let $\alpha$ be an automorphism of $F$; for each object $\xi$ of $\mathcal{X}$ over a scheme $Z$ we are given an automorphism $\alpha_{\xi}$ of $F(\xi)$ over $Z$, satisfying the usual condition for being a natural transformation. We are going to need the following two facts.

(a) If $\xi \rightarrow \zeta$ is a morphism in $\mathcal{X}$, then if $\alpha_{\zeta}$ is trivial then also $\alpha_{\xi}$ is trivial. This follows immediately from the fact that $\mathcal{X}$ is a category fibered in groupoids (see 2.1(1b).

(b) If $Z^{\prime} \rightarrow Z$ is an étale surjective map of schemes and $\xi^{\prime}$ is the pullback of $\xi$ to $Z^{\prime}$, then if $\alpha_{\xi^{\prime}}$ is trivial then also $\alpha_{\xi}$ is trivial. This follows from the fact that the isomorphism functors of $\mathcal{Y}$ are sheaves in the étale topology (see 2.1(2ai)).

(3) Let $\tilde{X} \rightarrow \mathcal{X}$ be an étale cover, and call $\xi$ the corresponding object of $\mathcal{X}$ over $\tilde{X}$. The restriction of $\alpha_{\xi}$ to the open subscheme $\tilde{U}=U \times \mathcal{X} \tilde{X} \subseteq \tilde{X}$ is trivial, and $\tilde{U}$ is scheme-theoretically dense in $\tilde{X}$; applying this lemma in the case of algebraic spaces it follows that $\alpha_{\xi}$ is trivial.

(4) Take an arbitrary object $\tau$ of $\mathcal{X}$ over a scheme $T$; then there is an étale cover $T^{\prime} \rightarrow T$ such that the pullback $\tau^{\prime}$ of $\tau$ to $T^{\prime}$ admits a morphism $\tau \rightarrow \xi$. Applying the two facts mentioned above, we get the result.

4.3. Twisted stable maps into a stack. As before, we consider a proper tame Deligne-Mumford stack $\mathcal{M}$ admitting a projective coarse moduli scheme $\mathbf{M}$. We fix an ample invertible sheaf on $\mathbf{M}$.

Definition 4.3.1. A twisted stable n-pointed map of genus $g$ and degree $d$ over $S$

$$
\left(\mathcal{C} \rightarrow S, \Sigma_{i}^{\mathcal{C}} \subset \mathcal{C}, f: \mathcal{C} \rightarrow \mathcal{M}\right)
$$

consists of a commutative diagram

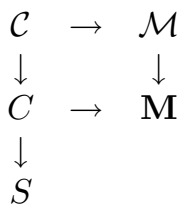

along with $n$ closed substacks $\Sigma_{i}^{\mathcal{C}} \subset \mathcal{C}$, satisfying:

(1) $\mathcal{C} \rightarrow C \rightarrow S$ along with $\Sigma_{i}^{\mathcal{C}}$ is a twisted nodal $n$-pointed curve over $S$;

(2) the morphism $\mathcal{C} \rightarrow \mathcal{M}$ is representable; and

(3) $\left(C \rightarrow S, \Sigma_{i}^{C}, f: C \rightarrow \mathbf{M}\right)$ is a stable $n$-pointed map of degree $d$.

Definition 4.3.2. A 1-morphism (or just a morphism) of twisted stable maps

$$
G:\left(\mathcal{C} \rightarrow S, \Sigma_{i}^{\mathcal{C}}, f: \mathcal{C} \rightarrow \mathcal{M}\right) \rightarrow\left(\mathcal{C}^{\prime} \rightarrow S^{\prime}, \Sigma_{i}^{\mathcal{C}^{\prime}}, f^{\prime}: \mathcal{C}^{\prime} \rightarrow \mathcal{M}\right)
$$


consists of data $G=(F, \alpha)$, where $F: \mathcal{C} \rightarrow \mathcal{C}^{\prime}$ is a morphism of twisted pointed curves, and $\alpha: f \rightarrow f^{\prime} \circ F$ is an isomorphism.

Twisted stable maps naturally form a 2-category. But by Proposition 4.2.2, this 2-category is equivalent to a category. We call this category the category of twisted stable maps.

\subsection{Equivalence of stable twisted objects and twisted stable maps.}

Theorem 4.4.1. The category of twisted stable maps is equivalent to the category of stable twisted objects, via an equivalence which preserves base schemes.

Proof. First part: construction of a functor from stable twisted objects to the category of twisted stable maps.

SteP 1: COnstruction of $\mathcal{C}$ Given $(\eta, C, \mathcal{A})$. Consider a stable twisted object $(\eta, C \rightarrow S, \mathcal{A})$ with $\mathcal{A}=\left\{\left(U_{\alpha}, \eta_{\alpha}, \Gamma_{\alpha}\right)\right\}$. For each pair of indices $(\alpha, \beta)$ let $R_{\alpha \beta}$ be the transition scheme from $\left(U_{\alpha}, \eta_{\alpha}, \Gamma_{\alpha}\right)$ to $\left(U_{\beta}, \eta_{\beta}, \Gamma_{\beta}\right)$. Let $U$ be the disjoint union of the $U_{\alpha}$, and let $R$ be the disjoint union of the $R_{\alpha \beta}$. The definition of $R_{\alpha \beta}$ via isomorphisms (see 3.3) implies that these have the following structure:

- there are two projections $R \rightarrow U$, which are étale;

- there is a natural diagonal morphism $U \rightarrow R$ which sends each $U_{\alpha}$ to $R_{\alpha \alpha}$; and

- there is a product $R \times_{U} R \rightarrow R$, sending each $R_{\alpha \beta} \times_{U_{\beta}} R_{\beta \gamma}$ to $R_{\alpha \gamma}$ via composition of isomorphisms.

These maps give $R \Longrightarrow U$ the structure of an étale groupoid, which defines a quotient Deligne-Mumford stack, which we denote by $\mathcal{C}$. This is obviously a nodal stack on $S$, and its moduli space is $C$. It is clear that $\mathcal{C} \rightarrow C$ is an isomorphism over $C_{\text {gen }}$. Also note that, étale locally over $C$, the stack $\mathcal{C}$ is isomorphic to $\left[U_{\alpha} / \Gamma_{\alpha}\right]$.

This construction depends on the atlas chosen, however we have:

Lemma 4.4.2. Let $\mathcal{A}^{\prime}$ and $\mathcal{A}^{\prime \prime}$ be two compatible atlases on a generic object $(\xi, C)$. Then the stacks $\mathcal{C}^{\prime}, \mathcal{C}^{\prime \prime}$ associated to the corresponding twisted objects are canonically isomorphic.

Proof. In fact, let $\mathcal{A}$ be the union of $\mathcal{A}^{\prime}$ and $\mathcal{A}^{\prime \prime}$. Let $R^{\prime} \Longrightarrow U^{\prime}, R^{\prime \prime} \Longrightarrow U^{\prime \prime}$ and $R \rightrightarrows U$ be the groupoids constructed from these three atlases, and let $\mathcal{C}^{\prime}, \mathcal{C}^{\prime \prime}$ and $\mathcal{C}$ be the quotient stacks. There are obvious embeddings of $R^{\prime} \Longrightarrow U^{\prime}$ and $R^{\prime \prime} \Longrightarrow U^{\prime \prime}$ into $R \Longrightarrow U$ inducing isomorphisms of $\mathcal{C}^{\prime}$ and $\mathcal{C}^{\prime \prime}$ with $\mathcal{C}$; by composing the isomorphism $\mathcal{C}^{\prime} \simeq \mathcal{C}$ with the inverse of $\mathcal{C}^{\prime \prime} \simeq \mathcal{C}$ we obtain the desired canonical isomorphism $\mathcal{C}^{\prime} \simeq \mathcal{C}^{\prime \prime}$.

Step 2: COnstruction of $\Sigma_{i}^{\mathcal{C}}$. Since, given two indices $\alpha, \beta$, the inverse image of $\Sigma_{i}^{U_{\alpha}}$ in $R_{\alpha \beta}$ coincides with the inverse image of $\Sigma_{i}^{U_{\beta}}$, the collection of the $\Sigma_{i}^{U_{\alpha}}$ defines a closed substack $\Sigma_{i}^{\mathcal{C}}$ of $\mathcal{C}$. Since the $\Sigma_{i}^{U_{\alpha}}$ are étale over $S$, it follows that $\Sigma_{i}^{\mathcal{C}}$ is étale over $S$; furthermore, the moduli space of $\Sigma_{i}^{\mathcal{C}}$ is $\Sigma_{i}^{C}$, so for any algebraically closed field $\Omega$ the induced functor $\Sigma_{i}^{\mathcal{C}}(\operatorname{Spec} \Omega) \rightarrow \Sigma_{i}^{C}(\operatorname{Spec} \Omega)$ induces a bijection of the set of isomorphism classes in $\Sigma_{i}^{\mathcal{C}}(\operatorname{Spec} \Omega)$ and $\Sigma_{i}^{C}(\operatorname{Spec} \Omega)$. This means that $\Sigma_{i}^{\mathcal{C}}$ is an étale gerbe over $S$, and $\mathcal{C} \rightarrow S$ has the structure of a twisted $n$-pointed curve.

If we start from a different, but compatible, atlas, the isomorphism between the two twisted curves constructed above preserves these markings.

Step 3: Construction of $O b(\mathcal{C}) \rightarrow O b(\mathcal{M})$. Putting together the objects $\eta_{\alpha}$ we have an object $\eta$ on $U$, and the tautological isomorphism between the two 
pullbacks of $\eta_{\alpha}$ and $\eta_{\beta}$ to $R_{\alpha \beta}$ yield an isomorphism $\phi$ of the two pullbacks of $\eta$ to $R$. Fix a morphism $T \rightarrow \mathcal{C}$, where $T$ is a scheme, and set $T^{\prime}=T \times_{\mathcal{C}} U, T^{\prime \prime}=T \times_{\mathcal{C}} R$. Denote by $\zeta^{\prime}$ the pullback of $\eta$ to $T^{\prime}$; then $\phi$ induces an isomorphism of the two pullbacks of $\zeta^{\prime}$ to $T^{\prime \prime}$ satisfying the cocycle condition. By descent for objects of $\mathcal{M}$ (see 2.1(2b) ), this isomorphism allows $\zeta^{\prime}$ to descend to an object $\zeta$ of $\mathcal{M}(T)$, or equivalently a morphism $T \rightarrow \mathcal{M}$. Thus we have associated to an object of $\mathcal{C}$ an object of $\mathcal{M}$.

Step 4: Construction of $\operatorname{Mor}(\mathcal{C}) \rightarrow \operatorname{Mor}(\mathcal{M})$. We need to associate to each arrow in $\mathcal{C}$ an arrow in $\mathcal{M}$ in such a manner that we get a morphism of stacks $\mathcal{C} \rightarrow \mathcal{M}$.

Consider an arrow in $\mathcal{C}$ over a morphism of schemes $T_{1} \rightarrow T_{2}$, which corresponds to the following commutative diagram:

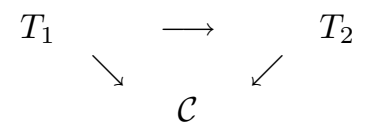

Set $T_{i}^{\prime}=T_{i} \times_{\mathcal{C}} U, T_{i}^{\prime \prime}=T_{i} \times_{\mathcal{C}} R$. Denote by $\zeta_{i}^{\prime}$ the pullback of $\eta$ to $T_{i}^{\prime}, \zeta_{i}$ the object obtained by descent to $T_{i}$; there is an arrow $\zeta_{1}^{\prime} \rightarrow \zeta_{2}^{\prime}$, together with descent data, inducing an arrow $\zeta_{1} \rightarrow \zeta_{2}$.

Thus we have a morphism of stacks $\mathcal{C} \rightarrow \mathcal{M}$.

If we start from two different but compatible atlases $\mathcal{A}^{\prime}$ and $\mathcal{A}^{\prime \prime}$ on the same generic object, we obtain two morphisms $\mathcal{C}^{\prime} \rightarrow \mathcal{M}$ and $\mathcal{C}^{\prime \prime} \rightarrow \mathcal{M}$. It is easily seen that there is a canonical isomorphism between this morphism $\mathcal{C}^{\prime} \rightarrow \mathcal{M}$ and the morphism $\mathcal{C}^{\prime} \rightarrow \mathcal{M}$ obtained by composing the isomorphism $\mathcal{C}^{\prime} \simeq \mathcal{C}^{\prime \prime}$ constructed above with the morphism $\mathcal{C}^{\prime \prime} \rightarrow \mathcal{M}$.

Step 5: The MORPhism $\mathcal{C} \rightarrow \mathcal{M}$ is RePresentable. This is a consequence of the fact that the action of the finite groups appearing in the charts of the atlas is essential, because of the following well-known lemma.

Lemma 4.4.3. Let $g: \mathcal{G} \rightarrow \mathcal{F}$ be a morphism of Deligne-Mumford stacks. The following two conditions are equivalent:

(1) The morphism $g: \mathcal{G} \rightarrow \mathcal{F}$ is representable.

(2) For any algebraically closed field $k$ and any $\xi \in \mathcal{G}(k)$, the natural group homomorphism $\operatorname{Aut}(\xi) \rightarrow \operatorname{Aut}(g(\xi))$ is a monomorphism.

Proof. By definition, the morphism $g: \mathcal{G} \rightarrow \mathcal{F}$ is representable if and only if the following condition holds:

- For any algebraic space $X$ and any morphism $f: X \rightarrow \mathcal{F}$, the stack $\mathcal{Y}:=$ $\mathcal{G} \times{ }_{\mathcal{F}} X$ is equivalent to an algebraic space.

For fixed $f: X \rightarrow \mathcal{F}$ the latter condition is equivalent to the following:

- The diagonal morphism $\Delta: \mathcal{Y} \rightarrow \mathcal{Y} \times \mathcal{Y}$ is a monomorphism.

This means:

- Given an algebraically closed field $k$, and an element $q \in \mathcal{Y} \times \mathcal{Y}(k)$ and two elements $p_{i} \in \mathcal{Y}(k)$, with isomorphisms $\beta_{i}: \Delta\left(p_{i}\right) \rightarrow q$, there exists a unique isomorphism $\phi: p_{1} \rightarrow p_{2}$ such that $\beta_{1}=\beta_{2} \circ \Delta(\phi)$.

We can write

$$
q=\left(\left(\mathfrak{g}_{1}, x_{1}, \alpha_{1}\right),\left(\mathfrak{g}_{2}, x_{2}, \alpha_{2}\right)\right)
$$


where $\mathfrak{g}_{i} \in \mathcal{G}(k), x_{i} \in X(k)$, and $\alpha_{i}: g\left(\mathfrak{g}_{i}\right) \stackrel{\sim}{\rightarrow} f\left(x_{i}\right)$. Similarly $p_{i}=\left(\mathfrak{g}_{i}^{\prime}, x_{i}^{\prime}, \alpha_{i}^{\prime}\right)$ as above. The existence of $\beta_{i}$ implies $x_{1}=x_{2}=x_{1}^{\prime}=x_{2}^{\prime}$. Also, composing with the given isomorphisms we may reduce to the case that, in fact, the same is true for the $\mathfrak{g}_{i}, \mathfrak{g}_{i}^{\prime}, \alpha_{i}$ and $\alpha_{i}^{\prime}$. Thus the condition above is equivalent to saying that for any $\mathfrak{g} \in G(k)$ there is a unique $\beta \in \operatorname{Aut}(\mathfrak{g})$ such that $g(\beta)=i d_{g(\mathfrak{g})}$, which is what we wanted.

Now, let $q \in \mathcal{M}($ Spec $k$ ), where $k$ is an algebraically closed field, and lift $q$ to a geometric point $p \in U(\operatorname{Spec} k)$; let $\alpha$ be such that $p \in U_{\alpha}$. The automorphism group of $q$ in $\mathcal{C}$ is the stabilizer of $p$ inside $\Gamma_{\alpha}$; the fact that this acts faithfully on the fiber of $\eta_{\alpha}$ over $p$ means exactly that this automorphism group injects inside the automorphism group of the image of $q$ in $\mathcal{M}$.

Step 6: Stability. The map $C \rightarrow S$ induced by this morphism $\mathcal{C} \rightarrow S$ is the map induced by the twisted object $(\xi, C \rightarrow S, \mathcal{A})$, and so is stable; this way we get a twisted stable map $\mathcal{C} \rightarrow \mathcal{M}$.

STEP 7: CONSTRUCTION OF THE MORPHISM OF TWISTED STABLE MAPS INDUCED BY A MORPHISM OF TWISTED OBJECTS. Let $f:\left(\xi_{1}, C_{1} \rightarrow S_{1}, \mathcal{A}_{1}\right) \rightarrow$ $\left(\xi_{2}, C_{2} \rightarrow S_{2}, \mathcal{A}_{2}\right)$ be a morphism of twisted objects, and let $\zeta_{1}: \mathcal{C}_{1} \rightarrow \mathcal{M}$ and $\zeta_{2}: \mathcal{C}_{2} \rightarrow \mathcal{M}$ be the corresponding twisted stable maps. Denote also by $\zeta_{1}^{\prime}: \mathcal{C}_{1}^{\prime} \rightarrow \mathcal{M}$ the twisted stable map associated to the twisted object $\left(\xi_{1}, C_{1} \rightarrow S_{1}, f^{*} \mathcal{A}_{2}\right)$. Since, as we have seen, there is a canonical isomorphism between $\zeta_{1}$ and $\zeta_{1}^{\prime}$, we are reduced to the case $\mathcal{A}_{1}=f^{*} \mathcal{A}_{2}$. In this case there is a morphism of groupoids $R_{1} \Longrightarrow U_{1}$ to $R_{1} \rightrightarrows U_{1}$, which induces a morphism of stacks $\mathcal{C}_{1} \rightarrow \mathcal{C}_{2}$.

This completes the definition of the functor from twisted objects to twisted stable maps.

Second part: construction of a functor from twisted stable maps to twisted objects.

Step 1: COnstruction of $(\eta, C, \mathcal{A})$. Let us take a twisted stable map $\mathcal{C} \rightarrow \mathcal{M}$, with moduli space $C \rightarrow S$. Since $C_{\text {gen }}$ is isomorphic to its inverse image in $\mathcal{C}$, the map $\mathcal{C} \rightarrow \mathcal{M}$ induces a map $C_{\text {gen }} \rightarrow \mathcal{M}$, and correspondingly a generic object $\xi$ on $C_{\text {gen }}$.

By Lemma 2.2.3, there is an étale covering $\left\{C_{\alpha} \rightarrow C\right\}$, such that for each $\alpha$ there is a scheme $U_{\sigma}$ and a finite group $\Gamma_{\alpha}$ acting on $U_{\alpha}$, with the property that the pullback $\mathcal{C} \times{ }_{C} C_{\alpha}$ is isomorphic to the stack-theoretic quotient $\left[U_{\alpha} / \Gamma_{\alpha}\right]$.

For each $i=1, \ldots, n$ we define $\Sigma_{i}^{U_{\alpha}}$ as the pullback of $\Sigma_{i}^{\mathcal{C}}$ to $U_{\alpha}$; then the action of the groups $\Gamma_{\alpha}$ preserves the subschemes $\Sigma_{i}^{U_{\alpha}} \subseteq C$. These subschemes are obviously disjoint and contained in the smooth locus of the projection $U_{\alpha} \rightarrow S$, so $U_{\alpha} \rightarrow S$ is an $n$-marked curve.

If we call $\eta_{\alpha}$ the object of $\mathcal{M}\left(U_{\alpha}\right)$ corresponding to the morphism $U_{\alpha} \rightarrow \mathcal{M}$, the action of $\Gamma_{\alpha}$ on $U_{\alpha}$ lifts to an action of $\Gamma_{\alpha}$ on $\eta_{\alpha}$. We get charts $\left(U_{\alpha}, \eta_{\alpha}, \Gamma_{\alpha}\right)$ for the generic object $\xi$. The fiber product $U_{\alpha} \times_{\mathcal{C}} U_{\beta}$ is étale over $U_{\alpha}$ and $U_{\beta}$. Since the given morphism $\mathcal{C} \rightarrow \mathcal{M}$ is representable and separated we have that $U_{\alpha} \times_{\mathcal{C}} U_{\beta}$ is a closed subspace of

$$
U_{\alpha} \times_{\mathcal{M}} U_{\beta}=\operatorname{Isom}_{U_{\alpha} \times{ }_{S} U_{\beta}}\left(\operatorname{pr}_{\alpha}^{*} \eta_{\alpha}, \operatorname{pr}_{\beta}^{*} \eta_{\beta}\right),
$$

where $\operatorname{pr}_{\alpha}$ and $\operatorname{pr}_{\beta}$ denote the projections of $U_{\alpha} \times_{S} U_{\beta}$ onto the two factors. This implies that $U_{\alpha} \times{ }_{\mathcal{C}} U_{\beta}$ is the transition scheme of the two charts, which are therefore compatible. It follows that we have defined an atlas on $C \rightarrow S$, and so a twisted 
object. The induced morphism $C \rightarrow \mathbf{M}$ is the one induced by $\mathcal{C} \rightarrow \mathcal{M}$, so it is stable.

If we start from a different covering of $\mathcal{C}$ by $\left[U_{\alpha} / \Gamma_{\alpha}\right]$ we get a canonically isomorphic twisted object. In fact, we essentially get the same twisted object with a different atlas.

Step 2: Construction of Morphisms. Given a morphism from $\mathcal{C}_{1} \rightarrow \mathcal{M}$ to $\mathcal{C}_{2} \rightarrow \mathcal{M}$, we automatically get a morphism $C_{1} \rightarrow C_{2}$. This association is obviously compatible with composition of morphisms.

Since $\mathcal{C}_{1}$ and $\mathcal{C}_{2}$ are tame, the formation of their moduli spaces $C_{1}$ and $C_{2}$ commute with base change (Lemma 2.3.3), so that $C_{1}=S_{1} \times_{S_{2}} C_{2}$. Since $\mathcal{C}_{1} \rightarrow \mathcal{C}_{2}$ is representable, we have that $\mathcal{C}_{1} \rightarrow C_{1} \times{ }_{C_{2}} \mathcal{C}_{2}$ is representable. Since this is an isomorphism over $\left(C_{1}\right)_{\text {gen }}$ and the geometric fibers of $C_{1} \times{ }_{C_{2}} \mathcal{C}_{2}$ are seminormal, we have that $\mathcal{C}_{1}$ is isomorphic to $C_{1} \times_{C_{2}} \mathcal{C}_{2}$, and the morphism $\mathcal{C}_{1} \rightarrow \mathcal{M}$ is isomorphic to the composite $\mathcal{C}_{1} \rightarrow \mathcal{C}_{2} \rightarrow \mathcal{M}$. From this it follows immediately that the pullback of a chart $(U, \eta, \Gamma) \in \mathcal{A}_{2}$ is compatible with all charts in $\mathcal{A}_{1}$.

Third part: proving that the two functors defined above are inverse to each other.

First of all start from a twisted map $\mathcal{C} \rightarrow \mathcal{M}$, and call $(\eta, C, \mathcal{A})$ the associated twisted object. This is obtained by taking a covering of $\mathcal{C}$ via $U_{\alpha}$ as in part 2; if we call $U$ the disjoint union of the $U_{\alpha}$, and let $R=U \times_{\mathcal{C}} U$, then the twisted curve associated with $(\eta, C, \mathcal{A})$ is the quotient of the groupoid $R \Longrightarrow U$, which is (canonically isomorphic to) $\mathcal{C}$. It is straightforward to check that the morphism $\mathcal{C} \rightarrow \mathcal{M}$ obtained from $(\eta, C, \mathcal{A})$ is canonically isomorphic to the given one.

Now start from a stable twisted object $(\eta, C, \mathcal{A})$, and consider the associated twisted stable map $\mathcal{C} \rightarrow \mathcal{M}$. The atlas $\mathcal{A}=\left\{\left(\eta_{\alpha}, U_{\alpha}, \Gamma_{\alpha}\right)\right\}$ yields a covering of $\mathcal{C}$ with stacks $\left[U_{\alpha} / \Gamma_{\alpha}\right]$; the associated twisted object is canonically isomorphic to $(\eta, C, \mathcal{A})$.

Remark 4.4.4. We draw attention to the following counter-intuitive phenomenon: given a twisted stable map $f: \mathcal{C} \rightarrow \mathcal{M}$, we can look at the automorphisms in Aut $_{C} \mathcal{C}$. Since $\mathcal{C}_{\text {gen }}=C_{\text {gen }}$, such an automorphism is a local object, dictated by the structure of $\mathcal{C}$ along the special locus of $C$. However, if such an automorphism comes from an automorphism of $f: \mathcal{C} \rightarrow \mathcal{M}$, then it is determined by the associated automorphism of the generic object $\xi$ on $C$. The structure of $\mathrm{Aut}_{C} \mathcal{C}$ is detailed in $\aleph-\mathrm{C}-\mathrm{V}$.

\section{The CATEGORY $\mathcal{K}_{g, n}(\mathcal{M}, d)$ IS AN ALgebraiC StACK}

\subsection{The stack axioms.}

Proposition 5.1.1. The category $\mathcal{K}_{g, n}(\mathcal{M}, d)$ is a limit-preserving stack, fibered by groupoids over $\mathcal{S} c h / \mathbb{S}$.

Proof. Condition 2.1(1): By definition $\mathcal{K}_{g, n}(\mathcal{M}, d)$ is fibered by groupoids over $\mathcal{S} c h / \mathbb{S}$, since there are pullbacks, and since all the morphisms of objects are given by fiber diagrams.

Condition 2.1(3A): It is also not difficult to see that $\mathcal{K}_{g, n}(\mathcal{M}, d)$ is limit preserving: given a twisted object $(\xi, C, \mathcal{A})$ over $\operatorname{Spec} R$, where $R=\lim _{\longrightarrow} R_{i}$, we may replace $\mathcal{A}$ by an atlas with finitely many charts if necessary; then the schemes $C, U_{\alpha}$ are of finite presentation, and therefore they come from some $\operatorname{Spec} R_{i}$; since the stack $\mathcal{M}$ is limit preserving, the objects $\eta_{\alpha}$ come from Spec $R_{i^{\prime}}$ for some $i^{\prime} \geq i$. 
Condition 2.1 (2b): We need to show that $\mathcal{K}_{g, n}(\mathcal{M}, d)$ has effective étale descent for objects.

Given a scheme $S$, an étale cover $\left\{S_{\alpha} \rightarrow S\right\}$, and twisted objects $\left(\xi_{\alpha}, C_{\alpha} \rightarrow\right.$ $\left.S_{\alpha}, \mathcal{A}_{\alpha}\right)$ together with isomorphisms between the pullbacks of $\left(\xi_{\alpha}, C_{\alpha} \rightarrow S_{\alpha}, \mathcal{A}_{\alpha}\right)$ and $\left(\xi_{\beta}, C_{\beta} \rightarrow S_{\beta}, \mathcal{A}_{\beta}\right)$ to $S_{\alpha} \times{ }_{S} S_{\beta}$ satisfying the cocycle condition, we claim that these descend to a twisted object $(\xi, C \rightarrow S, \mathcal{A})$ on $S$. The existence of the projective curve $C \rightarrow S$ is immediate from the sheaf axiom for $\mathcal{K}_{g, n}(\mathbf{M}, d)$. Now, any chart $\left(U_{\alpha}, \eta_{\alpha}, \Gamma_{\alpha}\right)$ for $\left(\xi_{\alpha}, C_{\alpha} \rightarrow S_{\alpha}\right)$ is also automatically a chart for $(\xi, C \rightarrow S)$. The charts coming from one $\left(\xi_{\alpha}, C_{\alpha} \rightarrow S_{\alpha}\right)$ are obviously compatible. Compatibility of the charts coming from $\left(\xi_{\alpha}, C_{\alpha} \rightarrow S_{\alpha}\right)$ and $\left(\xi_{\beta}, C_{\beta} \rightarrow S_{\beta}\right)$ follows since they are compatible when pulled back to $S_{\alpha} \times{ }_{S} S_{\beta}$.

CONDITION2.12(2a) and (3a): The other sheaf axiom requires each Isom functor to be a sheaf in the étale topology. Since we would like to show that $\mathcal{K}_{g, n}(\mathcal{M}, d)$ is an algebraic stack, we might as well prove that the Isom functor is representable:

Proposition 5.1.2. For any pair of stable twisted objects

$$
\tau_{1}=\left(\xi_{1}, C_{1} \rightarrow S, \mathcal{A}_{1}\right) \quad \text { and } \quad \tau_{2}=\left(\xi_{2}, C_{2} \rightarrow S, \mathcal{A}_{2}\right)
$$

over the same scheme $S$, the functor $\operatorname{Isom}_{S}\left(\tau_{1}, \tau_{2}\right)$ of isomorphisms of twisted objects is representable by a separated scheme of finite type over $S$.

Proof. Step 1: Reduction to the Case $C_{1}=C_{2}$. Consider the associated maps $\phi_{i}: C_{i} \rightarrow \mathbf{M}$; there is a natural transformation $\operatorname{Isom}_{S}\left(\tau_{1}, \tau_{2}\right) \rightarrow \operatorname{Isom}_{S}\left(\phi_{1}, \phi_{2}\right)$, where the second functor is the one associated with the diagonal in the stack $\mathcal{K}_{g, n}(\mathbf{M}, d)$. Since the second functor is known to be representable by separated schemes of finite type, it is enough to prove that this natural transformation is representable by separated schemes of finite type. This means that we can assume that $C_{1}$ is equal to $C_{2}$, and reformulate the problem as follows: if $\tau_{1}=\left(\xi_{1}, C \rightarrow S, \mathcal{A}_{1}\right)$ and $\tau_{2}=\left(\xi_{2}, C \rightarrow S, \mathcal{A}_{2}\right)$ are stable twisted objects over the same nodal curve $C \rightarrow S$, then the functor $I$ of isomorphisms of the two twisted objects inducing the identity on $C$ is representable by a separated scheme of finite type over $S$.

Step 2: Reduction to THE CASE Where $\xi_{1}=\xi_{2}$. Consider the scheme $\operatorname{Isom}_{C \text { gen }}\left(\xi_{1}, \xi_{2}\right)$, which is finite over $C_{\text {gen }}$; it can be extended, to a scheme $J$ finite over $C$, as follows.

Let $\mathcal{C}_{1}$ and $\mathcal{C}_{2}$ be the twisted curves underlying the twisted stable maps associated to $\xi_{1}, \xi_{2}$. Then the scheme

$$
\operatorname{Isom}_{C \text { gen }}\left(\xi_{1}, \xi_{2}\right)=C_{\text {gen }} \underset{\mathcal{M} \times C}{\times} C_{\text {gen }}
$$

is open inside the stack

$$
\mathcal{C}_{1} \underset{\mathcal{M} \times C}{\times} \mathcal{C}_{2} .
$$

Let $J$ be the coarse moduli space of the latter stack. Then $J$ is finite over $C$, and

$$
\operatorname{Isom}_{C \text { gen }}\left(\xi_{1}, \xi_{2}\right) \subset J
$$

is open.

By definition, each morphism of twisted objects $\tau_{1} \rightarrow \tau_{2}$ induces a section $C \rightarrow J$; this defines a morphism from $I$ to the Weil restriction $\mathrm{R}_{C / S} J$, which is quasiprojective over $S$ since $C \rightarrow S$ is projective and $J \rightarrow C$ is finite (see [G-FGA]). It is enough to prove that the morphism $I \rightarrow \mathrm{R}_{C / S} J$ is representable, separated and of finite type, so we may assume that $\xi_{1}$ is equal to $\xi_{2}$. 
SteP 3: THE CASE $\xi_{1}=\xi_{2}$. We see that the following lemma implies the thesis.

Lemma 5.1.3. Let $(\xi, C \rightarrow S)$ be a generic object with two atlases $\mathcal{A}_{1}$ and $\mathcal{A}_{2}$. Then there exists a closed subscheme $S^{\prime} \subseteq S$ such that given a morphism $T \rightarrow S$, the pullbacks of $\mathcal{A}_{1}$ and $\mathcal{A}_{2}$ to $T \times{ }_{S} C$ are compatible if and only if $T \rightarrow S$ factors through $S^{\prime}$.

Proof. We will prove a local version of the fact above.

Lemma 5.1.4. Let $(\xi, C \rightarrow S)$ be a generic object with two charts $(U, \eta, \Gamma)$ and $\left(U^{\prime}, \eta^{\prime}, \Gamma^{\prime}\right)$. Let $s_{0}$ be a geometric point of $S$; assume that the fiber of $C$ over $s_{0}$ has a unique special point $c_{0}$, and that there are two unique geometric points $u_{0} \in U$ and $u_{0}^{\prime} \in U^{\prime}$ over $c_{0}$. Then there exist an étale neighborhood $\tilde{S}$ of $s_{0}$ and a closed subscheme $S^{\prime} \subseteq \tilde{S}$ such that given a morphism $T \rightarrow \tilde{S}$, the pullbacks of the two charts to $T \times{ }_{\tilde{S}} C$ are compatible if and only if $T \rightarrow \tilde{S}$ factors through $S^{\prime}$.

Proof of the global Lemma 5.1.3 given the local Lemma 5.1.4. First fix a geometric point $s$ of $S$, and let $c_{1}, \ldots, c_{k}$ be the special points above it. For each $c_{i}$ let $\left(U_{i}, \eta_{i}, \Gamma_{i}\right)$ and $\left(U_{i}^{\prime}, \eta_{i}^{\prime}, \Gamma_{i}^{\prime}\right)$ be charts from $\mathcal{A}_{1}$ and $\mathcal{A}_{2}$. Refining the charts if necessary, we may assume that there are unique geometric points $u_{i}$ and $u_{i}^{\prime}$ in $U_{i}$ and $U_{i}^{\prime}$ over $c_{i}$. By Lemma 5.1.4 there exists an étale neighborhood $\tilde{S}_{i}$ of $s$ and a closed subscheme $S_{i}^{\prime} \subset \tilde{S}_{i}$ for compatibility of $\left(U_{i}, \eta_{i}, \Gamma_{i}\right)$ and $\left(U_{i}^{\prime}, \eta_{i}^{\prime}, \Gamma_{i}^{\prime}\right)$. We can choose a common refinement $\tilde{S}_{s}$ of $\tilde{S}_{i}$ such that the pullbacks of $U_{i}$ and $U_{i}^{\prime}$ cover all special points over $\tilde{S}_{s}$. Then the intersection $S_{s}^{\prime}=\bigcap S_{i}^{\prime} \times_{\tilde{S}_{i}} \tilde{S}_{s}$ is a closed subscheme of $\tilde{S}_{s}$ such that given a morphism $T \rightarrow \tilde{S}_{s}$, the pullbacks of the two atlases to $T \times_{\tilde{S}_{s}} X$ are compatible if and only if $T \rightarrow \tilde{S}_{s}$ factors through $S^{\prime}$.

Now choose a finite number of geometric points $s_{i}$ and étale neighborhoods $\tilde{S}_{s_{i}}$ which cover $S$. Applying Proposition 3.4.3, the union of the images of the closed subschemes $S_{s_{i}}^{\prime}$ is the closed subscheme required.

Proof of the local lemma. By passing to the fiber product $(U / \Gamma) \times_{C}\left(U^{\prime} / \Gamma^{\prime}\right)$ we may assume that $C=U / \Gamma=U^{\prime} / \Gamma^{\prime}$. By refining $C$ we may assume that $C$ and $U$ are affine, and that there exists an invariant effective Cartier divisor $D$ on $U$ containing the locus where the projection $U \rightarrow S$ is not smooth, but none of the fibers of $U \rightarrow S$. Using the local criterion for flatness, it is easy to see that such a divisor $D$ is flat.

By passing to an étale neighborhood of $s_{0}$ we can split $D$ into a number of connected components, so that the component containing $v_{0}$ is finite over $S$; then by deleting the other connected components we see that we may assume that $D$ is finite over $S$.

Consider the transition scheme $R$ of the two charts. There is a free action of the group $\Gamma^{\prime}$ over $R$; set $E=R / \Gamma^{\prime}$. The projection $E \rightarrow S$ is an isomorphism over the smooth locus of $C$, and is an isomorphism if and only if the projection $R \rightarrow U$ is étale. Take a morphism $T \rightarrow S$; then the transition schemes $R_{T}$ of the pullbacks of the two charts to $T$ is the scheme-theoretic closure of the inverse image of the smooth locus of $C$ in $R \times{ }_{S} T$, so $R_{T}$ is étale over $U \times{ }_{S} T$ if and only if the projection $\left(R \times{ }_{S} T\right) / \Gamma^{\prime}=E \times{ }_{S} T \rightarrow U \times{ }_{S} T$ has a section.

Set $S=\operatorname{Spec} \Lambda, U=\operatorname{Spec} A, E=\operatorname{Spec} B$, and call $I$ the ideal of $D$ in $A$. The coordinate ring of the complement of $D$ inside $U$ is $A^{\prime}=\bigcup_{i=0}^{\infty} I^{-i}$, and there is a natural homomorphism $B \rightarrow A^{\prime}$; given a $\Lambda$-algebra $L$ the coordinate ring of the quotient of the transition scheme of the pullbacks of the two charts to $\operatorname{Spec} L$ is the 
image of $B \otimes_{\Lambda} L$ in $A^{\prime} \otimes_{A} L$, so $\left(R \times_{S} T\right) / \Gamma^{\prime}=E \times_{S} T \rightarrow U \times_{S} T$ has a section if and only if the image of $B \otimes_{\Lambda} L$ in $A^{\prime} \otimes_{\Lambda} L$ is equal to $A \otimes_{\Lambda} L \subseteq A^{\prime} \otimes_{\Lambda} L$. Take a set of generators of $B$ as an $A$-algebra, and call $e_{1}, \ldots, e_{n}$ their images inside $A^{\prime} / A$; the condition that the image of $B \otimes_{\Lambda} L$ be equal to $A \otimes_{\Lambda} L$ is equivalent to the condition that the images of the $e_{i}$ in $\left(A^{\prime} / A\right) \otimes_{\Lambda} L$ be zero. Fix an integer $n$ such that the $e_{i}$ are all contained in $I^{-n} / A$; then the sequence

$$
0 \longrightarrow I^{-n} / A \longrightarrow A^{\prime} / A \longrightarrow \bigcup_{i=n}^{\infty} I^{-i} / I^{-n} \longrightarrow 0
$$

is exact and $\bigcup_{i=n}^{\infty} I^{-i} / I^{-n}=I^{-n} \otimes_{A}\left(A^{\prime} / A\right)$ is flat over $\Lambda$, so the sequence stays exact after tensoring with $L$. The conclusion is that $R_{T}$ is étale over $U \times{ }_{S} T$ if and only if the images of the $e_{i}$ in $\left(I^{-n} / A\right) \otimes_{\Lambda} L$ are zero. But $I^{-n} / A$ is finite and flat as a $\Lambda$-module, so it is projective, and can be embedded as a direct summand of a free $\Lambda$-module $F$. If $J \subseteq \Lambda$ is the ideal generated by the coefficients of the $e_{i}$ with respect to a basis of $F$, it is clear that the closed subscheme $S^{\prime}=\operatorname{Spec}(\Lambda / J)$ has the desired property.

This completes the proof of Proposition[5.1.2, and also of Proposition 5.1.1.

5.2. Base change. Artin's criteria for an algebraic stack work over a scheme of finite type over a field or an excellent Dedekind domain. Since $\mathcal{M} \rightarrow \mathbb{S}$ is of finite presentation, it is obtained by base change from a scheme of finite type over $\mathbb{Z}$. We need a similar statement of $\mathcal{K}_{g, n}(\mathcal{M}, d)$, which follows from the following result:

Proposition 5.2.1. Let $\mathbb{S}_{1} \rightarrow \mathbb{S}$ be a morphism of noetherian schemes and let $\mathcal{M} \rightarrow \mathbb{S}$ be as in the main theorem. Then $\mathcal{K}_{g, n}\left(\left(\mathcal{M} \times_{\mathbb{S}} \mathbb{S}_{1}\right) / \mathbb{S}_{1}, d\right)=\mathcal{K}_{g, n}(\mathcal{M}, d) \times_{\mathbb{S}}$ $\mathbb{S}_{1}$

Proof. Since $\mathcal{M}$ is tame, by Lemma 2.3.3 we have that $\mathbf{M} \times_{\mathbb{S}} \mathbb{S}_{1}$ is the moduli space of $\mathcal{M} \times_{\mathbb{S}} \mathbb{S}_{1}$. From this and Definition 4.3.1 the result is immediate.

5.3. Deformations and obstructions. So far we have seen that $\mathcal{K}_{g, n}(\mathcal{M}, d)$ is a limit-preserving stack, with separated representable diagonal of finite type. In order to show that it is algebraic, we need to produce an étale covering by a scheme $V \rightarrow \mathcal{K}_{g, n}(\mathcal{M}, d)$. We follow Artin's method, in which one starts from a deformation and obstruction theory, one constructs formal deformation spaces, and one shows they are algebraizable. We start by constructing a deformation and obstruction theory for $\mathcal{K}_{g, n}(\mathcal{M}, d)$. Here it is convenient to realize $\mathcal{K}_{g, n}(\mathcal{M}, d)$ as the category of twisted stable maps, rather than stable twisted objects.

Proposition 5.3.1. The category $\mathcal{K}_{g, n}(\mathcal{M}, d)$ has a deformation and obstruction theory satisfying conditions [2.1](3D) (i) through (iii).

Let $A_{0}$ be a reduced $\mathcal{O}_{\mathbb{S}}$-algebra of finite type, $\left(\mathcal{C}_{0} \rightarrow \operatorname{Spec} A_{0}, \Sigma_{0}, \mathcal{C}_{0} \rightarrow \mathcal{M}\right)$ a twisted stable map over $A_{0}$. For any $A_{0}$-module $I$ of finite type, let us call $\mathrm{D}(I)$ the set of isomorphism classes of twisted stable maps $\left(\mathcal{C} \rightarrow \operatorname{Spec}\left(A_{0}+I\right), \Sigma, \mathcal{C} \rightarrow \mathcal{M}\right)$, with a given isomorphism of the restriction of $\mathcal{C} \rightarrow \mathcal{M}$ to $A_{0}$ with $\mathcal{C}_{0} \rightarrow \mathcal{M}$. Recall that the set $\mathrm{D}(I)$ has a natural $A_{0}$-module structure. Denote by $\mathrm{L}$ the cotangent complex $\mathrm{L}_{\mathcal{C}_{0} / \mathcal{M} \times{ }_{S} \operatorname{Spec} A_{0}}$ (see [I] , II, 1.2.7, [L-MB], 17.3) and by $\mathcal{N}$ the normal sheaf of $\Sigma_{0}$ in $\mathcal{C}_{0}$. 
Lemma 5.3.2. There exists a canonical exact sequence $\mathrm{E}(I)$ of $A_{0}$-modules

$$
\operatorname{Hom}_{\mathcal{C}_{0}}\left(\mathrm{~L}, I \otimes A_{0} \mathcal{O}_{\mathcal{C}_{0}}\right) \rightarrow \mathrm{H}^{0}\left(\mathcal{C}_{0}, I \otimes \mathcal{N}\right) \rightarrow \mathrm{D}(I) \rightarrow \operatorname{Ext}^{1}\left(\mathrm{~L}, I \otimes_{A_{0}} \mathcal{O}_{\mathcal{C}_{0}}\right) \rightarrow 0
$$

which is functorial in $I$.

Proof. By [I], III, Proposition 2.1.2.3, the group $\mathrm{D}^{\prime}(I) \stackrel{\text { def }}{=} \operatorname{Ext}^{1}\left(\mathrm{~L}, I \otimes_{A_{0}} \mathcal{O}_{\mathcal{C}_{0}}\right)$ classifies extensions $\mathcal{C} \rightarrow \mathcal{M}$ of the twisted map $\mathcal{C}_{0} \rightarrow \mathcal{M}$, with the markings ignored. The natural map $\mathrm{D}(I) \rightarrow \mathrm{D}^{\prime}(I)$ is surjective, because $\Sigma_{0}$ is unobstructed inside $\mathcal{C}_{0}$, as $\mathrm{H}^{1}\left(\mathcal{C}_{0}, \mathcal{N}\right)=0$. The group $\mathrm{H}^{0}\left(\mathcal{C}_{0}, I \otimes_{A_{0}} \mathcal{N}\right)$ classifies extensions of $\Sigma_{0}$ inside the trivial extension $\mathcal{C}_{0} \times \operatorname{Spec} A_{0} \operatorname{Spec}\left(A_{0}+I\right)$, so $\mathrm{H}^{0}\left(\mathcal{C}_{0}, I \otimes_{A_{0}} \mathcal{N}\right)$ surjects onto the kernel of the map $\mathrm{D}(I) \rightarrow \mathrm{D}^{\prime}(I)$. Finally, $\operatorname{Hom}_{\mathcal{C}_{0}}\left(\mathrm{~L}, I \otimes_{A_{0}} \mathcal{O}_{\mathcal{C}_{0}}\right)$ is the group of $\mathcal{O}_{\mathcal{M}}$-derivations from $\mathcal{O}_{\mathcal{C}_{0}}$ into $I \otimes_{A_{0}} \mathcal{O}_{\mathcal{C}_{0}}$, therefore it is the group of infinitesimal automorphisms of $\mathcal{C}_{0} \times \operatorname{Spec} A_{0} \operatorname{Spec}\left(A_{0}+I\right)$ over $\mathcal{M}$ fixing $\mathcal{C}_{0}$, so it surjects onto the kernel of $\mathrm{H}^{0}\left(\mathcal{C}_{0}, I \otimes_{A_{0}} \mathcal{N}\right) \rightarrow \mathrm{D}(I)$.

Lemma 5.3.3. Let $A^{\prime} \rightarrow A \rightarrow A_{0}$ be infinitesimal extensions as in [Ar], so that $I=\operatorname{ker}\left(A^{\prime} \rightarrow A\right)$ is an $A_{0}$-module, and let $(\mathcal{C} \rightarrow \operatorname{Spec} A, \Sigma, \mathcal{C} \rightarrow \mathcal{M})$ be a twisted pointed map. The obstruction to lifting $(\mathcal{C} \rightarrow \operatorname{Spec} A, \Sigma, \mathcal{C} \rightarrow \mathcal{M})$ to $A^{\prime}$ lies in the group $\operatorname{Ext}_{\mathcal{O}_{\mathcal{C}}}^{2}\left(\mathrm{~L}_{\mathcal{C} / \mathcal{M}}, I \otimes_{A} \mathcal{O}_{\mathcal{C}}\right)$.

Proof. If there is an extension $\mathcal{C}^{\prime} \rightarrow \mathcal{M}$ of $\mathcal{C} \rightarrow \mathcal{M}$, then $\Sigma$ lifts to a subgerbe of $\mathcal{C}$, as indicated above. So we see that the obstruction to extending the twisted stable map coincides with the obstruction to extending $\mathcal{C} \rightarrow \mathcal{M}$. According to [I], III, Proposition 2.1.2.3, this obstruction is an element of $\operatorname{Ext}_{\mathcal{O}_{\mathcal{C}}}^{2}\left(\mathrm{~L}_{\mathcal{C} / \mathcal{M}}, I \otimes_{A} \mathcal{O}_{\mathcal{C}}\right)$.

Lemma 5.3.4. Let $A \rightarrow A_{0}$ be an infinitesimal extension of $A_{0}$, and let $\mathcal{C} \rightarrow$ Spec $A$ be a tame proper Deligne-Mumford stack. Let $\mathcal{L}$ be a complex bounded above, of sheaves of $\mathcal{O}_{\mathcal{C}}$-modules, with coherent cohomology. For each étale morphism $A \rightarrow B$ and each finite $B$-module $J$, set

$$
\mathrm{F}_{B}(J)=\operatorname{Ext}_{\mathcal{O}_{\mathcal{C}_{B}}}^{n}\left(\mathcal{L} \otimes_{A} B, J \otimes_{A} \mathcal{O}_{\mathcal{C}}\right)
$$

Let $I$ be a finite $A_{0}$-module. Then:

(1) If $A \rightarrow B$ is étale, then

$$
\mathrm{F}_{B}\left(I \otimes_{A_{0}} B_{0}\right)=\mathrm{F}_{A}(I) \otimes_{A_{0}} B_{0} .
$$

(2) If $\mathfrak{m}$ is a maximal ideal of $A$, then

$$
\mathrm{F}_{A_{0}}(I) \otimes A_{0} \underset{\longleftarrow}{\lim }\left(A / \mathfrak{m}^{k}\right)=\lim _{\longleftarrow} \mathrm{F}_{A_{0}}\left(I / \mathfrak{m}^{k} I\right) .
$$

(3) There is an open dense subset $U \subseteq \operatorname{Spec} A_{0}$ such that

$$
\mathrm{F}_{A_{0}}(I) \otimes_{A_{0}} k(p)=\mathrm{F}_{A_{0}}\left(I \otimes_{A_{0}} k(p)\right)
$$

for all $p \in U$.

Proof. Let $\mathcal{K}=\mathrm{RHom}_{\mathcal{O}_{\mathcal{C}}}\left(\mathcal{L}, \mathcal{O}_{\mathcal{C}_{0}}\right)$. Since $\mathcal{L}$ is bounded above, and locally quasiisomorphic to a complex of locally free sheaves, we have

$$
\mathrm{RHom}_{\mathcal{O}_{\mathcal{C}}}\left(\mathcal{L}, \mathcal{O}_{\mathcal{C}_{0}} \otimes_{A_{0}} I\right)=\mathcal{K} \otimes_{A_{0}}^{\mathrm{L}} I .
$$

By Lemma 2.3.4, the hypercohomology of the complex $\mathcal{K} \stackrel{\mathrm{L}}{\otimes}_{A_{0}} I$ is isomorphic to the hypercohomology of the complex of sheaves $\pi_{*}\left(\mathcal{K} \stackrel{\mathrm{L}}{\otimes}_{A_{0}} I\right)=\left(\pi_{*} \mathcal{K}\right) \stackrel{\mathrm{L}}{\otimes} A_{0} I$ on $C_{0} \subseteq C$. 
Statement (1) follows from the fact that formation of hypercohomology commutes with flat base change, for separated algebraic spaces; the case of a single sheaf is in $\mathrm{L}-\mathrm{MB}$, 13.1.9. From this one can deduce the general case by the usual spectral sequence argument.

Statement (2) can be deduced from the theorem on formal functions (see $\mathbf{K n}$, $\mathrm{V}, 3.1$ ). Note that each term in the complexes on the right is finite over the artinian ring $A_{0} / \mathrm{m}^{k}$, and therefore the complexes and their cohomologies satisfy the MittagLeffler condition. A standard spectral sequence argument (see [G-EGA], 0, 13.2.3) gives the statement.

Statement (3) is proved as follows. Since $A_{0}$ is reduced, by generic flatness we can localize, and assume that the cohomology sheaves of $\pi_{*} \mathcal{K} \stackrel{\mathrm{L}}{\otimes} A_{0} I$ are flat over $A_{0}$. By localizing further, we can also assume that the hypercohomology groups of $\pi_{*} \mathcal{K} \stackrel{\mathrm{L}}{\otimes} A_{0} I$ are projective over $A_{0}$. Then the statement follows from the standard base change theorem (see [B-G-I], IV, 3.10, and proof of [L-MB], 13.1.9).

We now come to our proof of Proposition 5.3.1.

Condition (i) follows from (1) in the lemma: for the obstructions it is immediate, and for the deformations it follows using the exact sequence $\mathrm{E}(I)$.

For (ii), first notice that $\lim _{\mathrm{E}} \mathrm{E}\left(I / \mathrm{m}^{k} I\right)$ is still exact, because each of the terms of $\mathrm{E}\left(I / \mathfrak{m}^{k} I\right)$ is an artinian $A_{0}$-module, and so they satisfy the Mittag-Leffler condition. The sequence $\mathrm{E}(I) \otimes_{A_{0}} \widehat{A}_{0}$ is also exact, because $\widehat{A}_{0}$ is flat over $A_{0}$, so the conclusion follows from (2) in the lemma above, together with the five lemma.

For (iii), note that there is a dense open set $U \subseteq \operatorname{Spec} A_{0}$ such that $\mathrm{E}(I) \otimes_{A_{0}} k(p)$ is exact for all $p$ in $U$. Then the result follows from part (3) of the lemma, and the five lemma.

5.4. Algebraization. To show that $\mathcal{K}_{g, n}(\mathcal{M}, d)$ is an algebraic stack, we need to verify conditions $2.1(3 \mathrm{~B})$ and $(3 \mathrm{C})$.

Condition (BCD) calls for Schlessinger's conditions as in [Ar], section 2, (S 1) and (S 2).

Condition (S 1), and even the stronger condition (S 1') (see $\underline{\mathrm{Ar}},(2.3)$ ), follows from standard principles. Indeed, given an infinitesimal extension $A^{\prime} \rightarrow A \rightarrow A_{0}$ and a ring homomorphism $B \rightarrow A$ such that $B \rightarrow A_{0}$ is surjective, and given stable twisted objects $\left(\xi_{A^{\prime}}, C_{A^{\prime}} \rightarrow \operatorname{Spec} A^{\prime}, \mathcal{A}_{A^{\prime}}\right)$ and $\left(\xi_{B}, C_{B} \rightarrow \operatorname{Spec} B, \mathcal{A}_{B}\right)$, there is a gluing $C \rightarrow$ Spec $A^{\prime} \times{ }_{A} B$, along with gluings of the schemes $U_{\alpha}$ underlying the charts. The objects $\eta_{\alpha}$ can be glued since the condition applies to $\mathcal{M}$.

The finiteness condition (S 2) follows from the properness of $\mathcal{C}$ and the exactness of the pushforward along $\mathcal{C} \rightarrow C$ (Lemma 2.3.4), because of the cohomological description of $\mathrm{D}(I)$.

We now come to condition (3B - that formal deformations are algebraizable.

Proposition 5.4.1. Let $A$ be a complete noetherian local ring over our base scheme $S$, with maximal ideal $\mathfrak{m}$. Set $A_{n}=A / \mathfrak{m}^{n+1}$. Let $\xi_{n}: \mathcal{C}_{n} \rightarrow \mathcal{M}$ be a twisted stable map over Spec $A_{n}$, together with isomorphisms $\alpha_{n}:\left.\xi_{n}\right|_{\text {Spec } A_{n-1}} \simeq \xi_{n-1}$. Then there exist a twisted stable map $\xi: \mathcal{C} \rightarrow \mathcal{M}$ over $\operatorname{Spec} A$ together with isomorphisms $\left.\xi\right|_{\text {Spec } A_{n}} \simeq \xi_{n}$, compatible with the $\alpha_{n}$.

Proof. Step 1: Construction of $C \rightarrow$ M. Let $\pi_{n}: \mathcal{C}_{n} \rightarrow C_{n}$ be the moduli space of $\mathcal{C}_{n}$. The fact that the stack $\mathcal{K}_{g, n}(\mathbf{M}, d)$ is algebraic insures the existence of 
a projective curve $C$ over $\operatorname{Spec} A$, together with isomorphisms $\left.C\right|_{\operatorname{Spec} A_{n}} \simeq C_{n}$ compatible with the isomorphisms $\left.C_{n}\right|_{\operatorname{Spec} A_{n-1}} \simeq C_{n-1}$, and a map $C \rightarrow \mathbf{M}$ extending the maps $C_{n} \rightarrow \mathbf{M}$.

Step 2: Construction of $\mathcal{C} \rightarrow \mathcal{M}$. The stack $\mathcal{M} \times{ }_{\mathbf{M}} C$ is proper and tame over $\operatorname{Spec} A$, and its restriction to $\operatorname{Spec} A_{n}$ is $\mathcal{M} \times{ }_{\mathbf{M}} C_{n}$. Consider the maps

$$
\eta_{n}=\xi_{n} \times \pi_{n}: \mathcal{C}_{n} \rightarrow \mathcal{M} \times{ }_{\mathrm{M}} C_{n} .
$$

The morphisms $\eta_{n}$ are representable and finite, so they define a sheaf of finite algebras $\eta_{n_{*}} \mathcal{O}_{\mathcal{C}_{n}}$ over $\mathcal{M} \times_{\mathrm{M}} C_{n}$. By the existence theorem A.1.1 given in the appendix, there is a sheaf $\mathcal{R}$ of finite $\mathcal{O}_{\mathcal{M} \times{ }_{M} C^{-a l g e b r a s ~ w i t h ~ c o m p a t i b l e ~ i s o m o r p h i s m s ~}}$ $\left.\mathcal{O}_{\mathcal{C}}\right|_{\text {Spec } A_{n}} \simeq \mathcal{O}_{\mathcal{C}_{n}} ;$ by $[\mathrm{L}-\mathrm{MB}$ ], Proposition 14.2.4, there is a finite representable map $\eta: \mathcal{C} \rightarrow \mathcal{M} \times{ }_{\mathrm{M}} C$ such that $\eta_{*} \mathcal{O}_{\mathcal{C}}=\mathcal{A}$.

By the local criterion of flatness ( $\overline{\mathrm{Ma}}$, Theorem 49 , condition 5$) \mathcal{C}$ is flat over $A$. By deformation theory, $\mathcal{C}$ is a nodal stack over $A$.

Let us show that the coarse moduli space of $\mathcal{C}$ is indeed $C$. Call $\bar{C}$ the coarse moduli space of $\mathcal{C}$, with the induced map $\bar{C} \rightarrow C$. This is finite. Because of the local criterion of flatness, it is flat (see [Ma], 20.G). Furthermore since the map $\left.\bar{C}\right|_{\text {Spec } A_{0}} \rightarrow C_{0}$ is an isomorphism outside the special locus of $C_{0}$, the map $\bar{C} \rightarrow C$ is finite, flat and of degree 1 , so it is an isomorphism.

SteP 3: CONSTRUCTION OF $\Sigma^{\mathcal{C}}$. Let $\Sigma^{\mathcal{C}_{n}} \subseteq \mathcal{C}_{n}$ be the union of the markings. Again by the existence theorem, there is a unique substack $\Sigma^{\mathcal{C}} \subseteq \mathcal{C}$ whose intersection with the $\mathcal{C}_{n}$ is $\Sigma^{\mathcal{C}_{n}}$. The stack $\Sigma^{\mathcal{C}}$ is flat over $\operatorname{Spec} A$, and is étale over the closed point, so it is étale. By the same argument, the diagonal is also étale. Therefore $\Sigma^{\mathcal{C}}$ is a gerbe over its moduli space, which is a union of disjoint sections of $C$.

Step 4: StruCture of $\mathcal{C} \rightarrow C$. We only have left to prove that the morphism $\mathcal{C} \rightarrow C$ is an isomorphism outside the union of $\Sigma^{\mathcal{C}}$ and the singular locus $\mathcal{C}_{\text {sing }}$ of the map $\mathcal{C} \rightarrow \operatorname{Spec} A$. There exists a closed substack of $\mathcal{C}$ where the inertia groups are nontrivial; this substack meets $\mathcal{C}_{0}$ at most at points of $\Sigma^{\mathcal{C}}$ and $\mathcal{C}_{\text {sing. }}$. We need to analyze the structure of $\mathcal{C}$ near a point of $\Sigma_{0}$ and near a point of $\mathcal{C}_{0} \cap \mathcal{C}_{\text {sing }}$.

Take a geometric point $p=\operatorname{Spec} \Omega \rightarrow \mathcal{C}_{0}$, and let $R$ be the completion of the strict henselization of $\mathcal{C}$ at $p$. Call $\Gamma$ the automorphism group of $p$; this is a cyclic group of order prime to the characteristic of $A_{0}$. Denote $R_{n}=R \otimes_{A} A_{n}$.

Case 1: $p \in \Sigma$.

Now $R_{0}$ is isomorphic to $A_{0}\left[\left[\xi_{0}\right]\right]$, where $\xi_{0}$ is an indeterminate which is a semiinvariant for $\Gamma$. The ideal generated by $\xi_{0}$ defines $\Sigma$ at $p$. Lift $\xi_{0}$ to a semi-invariant element $\xi \in R$. Then $R=A[[\xi]]$; we only need to check that the ideal generated by $\xi$ defines $\Sigma$. Denote the reduction of $\xi$ in $R_{n}$ by $\xi_{n}$. The ideal of $\Sigma_{n}$ in $R_{n}$ is generated by a semi-invariant $\zeta_{n}$, and it is easy to see that $\zeta_{n}=u \xi_{n}$, where $u \in R_{n}$ is an invariant unit. So $\xi_{n}$ also generates the ideal of $\Sigma_{n}$ in $R_{n}$, and this implies that $\xi$ generates the ideal of $\Sigma$ in $R$. This proves that $\mathcal{C}$ is isomorphic to $C$ outside $\Sigma$ in a neighborhood of each point of $\Sigma \cap \mathcal{C}_{0}$.

Case 2: $p \in \mathcal{C}_{\text {sing }}$.

This time $R_{0}$ is isomorphic to $A_{0}\left[\left[\xi_{0}, \eta_{0}\right]\right] /\left(\xi_{0} \cdot \eta_{0}\right)$. We can choose $\xi_{0}$ and $\eta_{0}$ semi-invariants. By deformation theory, there exists a lifting $\xi^{\prime}$ of $\xi_{0}$ and $\eta^{\prime}$ of $\eta_{0}$, such that $\xi^{\prime} \cdot \eta^{\prime} \in A$; in other words, $R=A\left[\left[\xi^{\prime}, \eta^{\prime}\right]\right] /\left(\xi^{\prime} \cdot \eta^{\prime}-a\right)$. Let $\xi$ be any semi-invariant lifting of $\xi_{0}$. Then $\xi=u \cdot \xi^{\prime}$ for some unit $u \in R$. If we denote $\eta^{\prime \prime}=u^{-1} \eta^{\prime}$, then we have $R=A\left[\left[\xi, \eta^{\prime \prime}\right]\right] /\left(\xi \cdot \eta^{\prime \prime}-a\right)$. Denote by $\chi_{\xi}$ and $\chi_{\eta}$ the 
characters by which $\Gamma$ acts on $\xi_{0}$ and $\eta_{0}$. Write

$$
\eta=\frac{1}{|\Gamma|} \sum_{\gamma \in \Gamma} \chi_{\eta}^{-1}(\gamma) \cdot \gamma \eta^{\prime \prime}
$$

Clearly $\eta$ is a semi-invariant lifting of $\eta_{0}$. If $a=0$, then clearly $\xi \cdot \eta=0$ and we are done. If, on the other hand, $a \neq 0$, then $\chi_{\eta}=\chi_{\xi}^{-1}$, and we have

$$
\begin{aligned}
a & =\frac{1}{|\Gamma|} \sum_{\gamma \in \Gamma} \gamma a=\frac{1}{|\Gamma|} \sum_{\gamma \in \Gamma} \gamma\left(\xi \eta^{\prime \prime}\right)=\frac{1}{|\Gamma|} \sum_{\gamma \in \Gamma} \chi_{\xi}(\gamma) \xi \cdot \gamma \eta^{\prime \prime} \\
& =\xi \frac{1}{|\Gamma|} \sum_{\gamma \in \Gamma} \chi_{\eta}^{-1}(\gamma) \cdot \gamma \eta^{\prime \prime}=\xi \eta .
\end{aligned}
$$

Again, this proves that $\mathcal{C}$ is isomorphic to $C$ outside $\mathcal{C}_{\text {sing }}$ in a neighborhood of each point of $\mathcal{C}_{\text {sing }} \cap \mathcal{C}_{0}$.

So the pair $\mathcal{C}, \Sigma$ with the map $\mathcal{C} \rightarrow \mathcal{M}$ gives a twisted stable map. This map is stable, since $C \rightarrow \mathbf{M}$ is stable.

Thus $\mathcal{K}_{g, n}(\mathcal{M}, d)$ is an algebraic stack. Since an automorphism of a twisted object $(\xi, C, \mathcal{A})$ fixing $C \rightarrow \mathbf{M}$ is determined by its action on the generic object $\xi$, and since $\mathcal{M}$ is a tame Deligne-Mumford stack, we obtain that $\mathcal{K}_{g, n}(\mathcal{M}, d) \rightarrow$ $\mathcal{K}_{g, n}(\mathbf{M}, d)$ is of Deligne-Mumford type and tame.

Remark 5.4.2. The proof of Case 2 above implies the following lemma, which will be used later (Proposition 8.1.1).

Lemma 5.4.3. Let $(\xi, C \rightarrow S, \mathcal{A})$ be a twisted object. Then there is an open and closed subscheme $T \subseteq S$ such that if $s_{0}$ is a geometric point of $S$, then the pullback of $(\xi, C \rightarrow S, \mathcal{A})$ to $s_{0}$ is balanced if and only if $s_{0}$ is in $T$.

\section{The Weak VAluative CRITERion}

We wish to show that $\mathcal{K}_{g, n}(\mathcal{M}, d)$ is proper and $\mathbf{K}_{g, n}(\mathcal{M}, d)$ is projective. We start by verifying the weak valuative criterion for $\mathcal{K}_{g, n}(\mathcal{M}, d)$.

Let $R$ be a discrete valuation $\operatorname{ring}, S=\operatorname{Spec}(R)$. Let $\eta \in S$ be the generic point, $s \in S$ the special point. For an injection of discrete valuation rings $R \subset R_{1}$, we denote by $S_{1}, \eta_{1}, s_{1}$ the corresponding schemes.

Proposition 6.0.1. Let $\left(\xi, C_{\eta} \rightarrow\{\eta\}, \mathcal{A}_{\eta}\right)$ be a stable twisted object. Then there is an injection of discrete valuation rings $R \subset R_{1}$ inducing a finite extension of fraction fields, and an extension

$$
\begin{aligned}
& \left(\xi \times_{S} S_{1}, C_{\eta} \times{ }_{S} S_{1}, \mathcal{A}_{\eta} \times{ }_{S} S_{1}\right) \subset\left(\xi_{1}, C_{1}, \mathcal{A}_{1}\right) \\
& \downarrow \downarrow \downarrow \\
& \left\{\eta_{1}\right\} \quad \subset \quad S_{1},
\end{aligned}
$$

that is, $\left(\xi_{1}, C_{1} \rightarrow S_{1}, \mathcal{A}_{1}\right)$ is a stable twisted object and its pullback to $\left\{\eta_{1}\right\}$ is isomorphic to the pullback of $\left(\xi, C_{\eta} \rightarrow\{\eta\}, \mathcal{A}_{\eta}\right)$ to $\left\{\eta_{1}\right\}$. The extension $\left(\xi_{1}, C_{1} \rightarrow\right.$ $\left.S_{1}, \mathcal{A}_{1}\right)$ is unique up to a unique isomorphism, and its formation commutes with further injections of discrete valuation rings. If $\left(\xi, C_{\eta} \rightarrow\{\eta\}, \mathcal{A}_{\eta}\right)$ is balanced, then $\left(\xi_{1}, C_{1} \rightarrow S_{1}, \mathcal{A}_{1}\right)$ is balanced as well.

Proof. We will proceed in steps.

Step 1: EXtension of $C$. Let $f_{\eta}: C_{\eta} \rightarrow \mathbf{M}$ be the coarse moduli morphism. By $\left[\mathrm{F}-\mathrm{P}\right.$ and $[\mathrm{B}-\mathrm{M}]$, we know that $\mathcal{K}_{g, n}(\mathbf{M}, d)$ is a proper algebraic stack. By 
the weak valuative criterion for $\mathcal{K}_{g, n}(\mathbf{M}, d)$, it follows that there is an injection of discrete valuation rings $R \subset R_{1}$, inducing a finite extension of fraction fields, and an extension

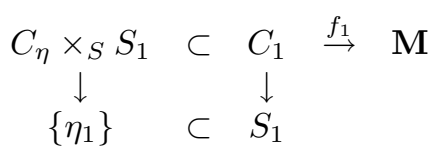

such that $f_{1}: C_{1} \rightarrow \mathbf{M}$ is a family of Kontsevich stable maps. The extension (3) is unique up to a unique isomorphism and commutes with further base changes.

We now replace $R$ by $R_{1}$. Let $\pi \in R$ be a uniformizer.

Step 2: extension of $C_{\eta} \rightarrow \mathcal{M}$ over $C_{\text {gen }}$. We may assume that over $\left(C_{\eta}\right)_{\text {gen }}$ we have a chart with $U_{\eta}=\left(C_{\eta}\right)_{\text {gen }}$ and trivial $\Gamma$ : such a chart is compatible with any other chart, so we may add it to the atlas. We may extend this via $U=C_{\text {gen }}$. However the map $C_{\eta} \rightarrow \mathcal{M}$ does not necessarily extend over $C_{\text {gen }}$, so we may need a further base change.

Let $C_{i}$ be the components of the special fiber $C_{s}$, and let $\zeta_{i} \in C_{i}$ be the generic points. Consider the localization $C_{\zeta_{i}}$. This is the spectrum of a discrete valuation ring. By the weak valuative criterion for $\mathcal{M}$, there is a tamely ramified quasifinite surjective cover $\tilde{C}_{i} \rightarrow C_{\zeta_{i}}$ and a map $\tilde{C}_{i} \rightarrow \mathcal{M}$ lifting the map on $\left(C_{\eta}\right)_{\text {gen }}$.

We proceed to simplify these schemes $\tilde{C}_{i}$. Denote $S_{n}=\operatorname{Spec} R\left[\pi^{1 / n}\right]$. By Abhyankar's lemma (G-SGA1], exp. XIII, section 5) we may assume that $\tilde{C}_{i}$ is étale over $C_{\zeta_{i}} \times_{S} S_{n_{i}}$. By descent we already have a lifting $C_{\zeta_{i}} \times_{S} S_{n_{i}} \rightarrow \mathcal{M}$. Taking $n$ divisible by all the $n_{i}$, we have an extension $C_{\zeta_{i}} \times S_{S} S_{n} \rightarrow \mathcal{M}$.

We now replace $R$ by $R\left[\pi^{1 / n}\right]$. Thus there is an extension $C_{\zeta_{i}} \rightarrow \mathcal{M}$. Note that this extension is unique, since $\mathcal{M}$ is separated. For the same reason it commutes with further base changes.

By dJ-O, there is a maximal open set $U \subset C_{\text {gen }}$ with an extension of the morphism, $U \rightarrow \mathcal{M}$. Since $U$ contains both $\left(C_{\eta}\right)_{\text {gen }}$ and $\zeta_{i}$, we have $U=C_{\text {gen }} \backslash P$ for a finite set of closed points $P$. By the purity lemma (Lemma 2.4.1) we have that $U=C_{\text {gen }}$, and there is an extension $C_{\text {gen }} \rightarrow \mathcal{M}$.

The uniqueness of the lifting in the purity lemma guarantees that this extension is unique up to a unique isomorphism, and commutes with further base changes.

SteP 3: extension of $C_{\eta} \rightarrow \mathcal{M}$ Over nongeneriC nodes. Let $p \in \operatorname{Sing}(C)$ be a node, and assume $p$ is not in the closure of $\operatorname{Sing}\left(C_{\eta}\right)$.

To build up a chart near $p$, we first choose an étale neighborhood $W \rightarrow C$ of $p$, as follows. Let $\bar{W}$ be a Zariski neighborhood such that $\bar{W} \cap \operatorname{Sing}(C)=\{p\}$. We already have a morphism $\bar{W} \backslash\{p\} \rightarrow \mathcal{M}$. We take an étale neighborhood $W$ of $p$ over $\bar{W}$ so that $p$ is a split node on $W$. Thus we can find elements $s_{1}, s_{2}$ in the maximal ideal of $p$, such that $\mathfrak{m}_{p}=\left(s_{1}, s_{2}, \pi\right)$, satisfying the equation $s_{1} s_{2}=\pi^{r}$ for some $r>1$. In other words, $W$ is also an étale neighborhood of the closed point $\left\{s_{1}=s_{2}=\pi=0\right\}$ in $W^{\prime}=\operatorname{Spec} R\left[s_{1}, s_{2}\right] /\left(s_{1} s_{2}-\pi^{r}\right)$.

Write $r=r_{1} r_{2}$, where $r_{2}$ is the maximal power of the residue characteristic in $r$, and thus $r_{1}$ is prime to the characteristic.

We now find a nodal curve $U_{0} \rightarrow S$, which is Galois over $W$, with an equivariant extension $U_{0} \rightarrow \mathcal{M}$. Let $U_{0}^{\prime} \rightarrow W^{\prime}$ be defined as follows:

$$
U_{0}^{\prime}=\operatorname{Spec} R\left[t_{1}, t_{2}\right] /\left(t_{1} t_{2}-\pi^{r_{2}}\right),
$$

and $s_{1}=t_{1}^{r_{1}}, s_{2}=t_{2}^{r_{1}}$. There is an obvious balanced action of the group of $r_{1}$-th roots of unity $\boldsymbol{\mu}_{r_{1}}$ on $U_{0}^{\prime}$ via $\left(t_{1}, t_{2}\right) \mapsto\left(\zeta t_{1}, \zeta^{-1} t_{2}\right)$, and $U_{0}^{\prime} \rightarrow W^{\prime}$ is the associated 
quotient morphism. Let $U_{0}=U_{0}^{\prime} \times_{W^{\prime}} W$. The action of $\boldsymbol{\mu}_{r_{1}}$ clearly lifts to $U_{0}$. We write $q_{0}: U_{0} \rightarrow W$ for the quotient map.

Notice that $U_{0}^{\prime}$, and therefore $U_{0}$, satisfies Serre's condition $S_{2}$. Also, $U_{0}^{\prime}$ (and therefore also $U_{0}$ ) has a homeomorphic nonsingular cover $\tilde{U}^{\prime}$ given by taking roots of order $r_{2}$ of $t_{1}$ and $t_{2}$. Thus the local fundamental group is trivial, and the purity lemma applies. By the purity lemma we have a lifting $U_{0} \rightarrow \mathcal{M}$ of the morphism $f \circ q_{0}: U_{0} \rightarrow \mathcal{M}$. Notice that for $g \in \boldsymbol{\mu}_{r_{1}}$ we have $f \circ q_{0}=f \circ q_{0} \circ g$. Thus by the uniqueness of the lifting, the map $U_{0} \rightarrow \mathcal{M}$ commutes with the action of $\boldsymbol{\mu}_{r_{1}}$.

Let $\eta_{0} \in \mathcal{M}\left(U_{0}\right)$ be the associated object and let $\eta_{p}$ be the fiber over $p$. The group $\boldsymbol{\mu}_{r_{1}}$ acts on $\eta_{0}$, stabilizing $\eta_{p}$. Let $\boldsymbol{\mu}_{r_{0}} \subset \boldsymbol{\mu}_{r_{1}}$ be the subgroup acting trivially on $\eta_{p}$. Denote by $U=U_{0} / \boldsymbol{\mu}_{r_{0}}$ and $\Gamma=\boldsymbol{\mu}_{r_{1}} / \boldsymbol{\mu}_{r_{0}}$ the quotients. By Lemma 2.5.1 there is a $\Gamma$-equivariant object $\eta: U \rightarrow \mathcal{M}$, and the action is essential.

Thus we have a chart $(U, \eta, \Gamma)$ near $p$. This is easily seen to be unique and to commute with further base changes. Note that the chart we have constructed is automatically balanced.

SteP 4: EXtension of CHARTS OVER Generic Nodes. Let $p_{\eta} \in \operatorname{Sing}\left(C_{\eta}\right)$, and let $p \in C_{s}$ be in the closure of $p_{\eta}$. The construction of a chart here is similar to step 2. We choose a Zariski neighborhood $\bar{W}$ of $p$ such that $\bar{W} \cap \operatorname{Sing}(C)=\left\{p_{\eta}, p\right\}$. As before, we may choose an étale neighborhood $W$ over $\bar{W}$ such that $p_{\eta}$ is a split node, thus $W$ is étale over $\left\{s_{1} s_{2}=0\right\}$. We may assume that $U_{\eta}$ is given by $t_{1} t_{2}=0$, where $t_{i}^{r}=s_{i}$ for an appropriate integer $r$ prime to the residue characteristic, with the group $\Gamma=\boldsymbol{\mu}_{r}$. Otherwise we can add a compatible chart with such a $U_{\eta}$ to our atlas. There is an obvious extension $U_{\eta} \subset U$ via the same equation $\left\{t_{1} t_{2}=0\right\}$, and the action of $\Gamma$ extends automatically. We already have a lifting $U \backslash\{p\} \rightarrow \mathcal{M}$. Since $U$ is normal crossings, the purity lemma applies, and guarantees that there is a unique equivariant extension to $U$. It is easy to check that this gives an extension of the chart, which is essential (as the Isom scheme of $\mathcal{M}$ is finite unramified). Also, if the chart on $\left(\xi, C_{\eta} \rightarrow S, \mathcal{A}_{\eta}\right)$ is balanced at $p_{\eta}$, then the chart we have constructed is evidently balanced as well.

Step 5: extension of $C_{\eta} \rightarrow \mathcal{M}$ over $\Sigma_{i}$. This step is identical to the previous one.

The uniqueness and base change properties are straightforward.

\section{BOUNDEDNESS}

To complete the proof of properness of $\mathcal{K}_{g, n}(\mathcal{M}, d)$, we need to show it is of finite type. Since $\mathcal{K}_{g, n}(\mathbf{M}, d)$ is of finite type, it suffices to show that the morphism $\mathcal{K}_{g, n}(\mathcal{M}, d) \rightarrow \mathcal{K}_{g, n}(\mathbf{M}, d)$ is of finite type. As a first step, we show in Theorem 7.1.1 that for a family of stable maps over a scheme $T$, there is a family of twisted stable maps over a scheme $T^{\prime}$ of finite type over $T$ which on geometric points exhausts all possible liftings of the given stable maps. The existence of algebraic deformation spaces allows one to capture the infinitesimal structure as well, showing that $\mathcal{K}_{g, n}(\mathcal{M}, d)$ is of finite type.

As it turns out, the difficult step in Theorem 7.1.1 is in showing that there is an exhaustive family of generic objects for a stable map with smooth source curve (Lemma 7.2.5). We show this in a sequence of lemmas by "explicitly" giving descent data for all such generic objects.

7.1. The statement. The bulk of this section is devoted to proving the following result: 
Theorem 7.1.1. Given a morphism $T \rightarrow \mathcal{K}_{g, n}(\mathbf{M}, d)$, where $T$ is a scheme of finite type over $\mathbb{S}$, there exists a morphism $T^{\prime} \rightarrow T$ of finite type, and a lifting $T^{\prime} \rightarrow \mathcal{K}_{g, n}(\mathcal{M}, d)$, such that a geometric point of $\mathcal{K}_{g, n}(\mathcal{M}, d)$ is in the image of $T^{\prime}$ if its image in $\mathcal{K}_{g, n}(\mathbf{M}, d)$ is in the image of $T$.

Let $C \rightarrow T$ be the corresponding underlying family of curves, with stable map $f: C \rightarrow \mathbf{M}$.

\subsection{The smooth case.}

Proposition 7.2.1. The theorem holds when $C \rightarrow T$ is smooth.

By noetherian induction, it suffices to prove the proposition after a dominant base change of finite type on $T$. In particular we may assume $T$ irreducible. Consider $\overline{f(C)}$, the closure of the image of $C$ in $\mathbf{M}$; by [L-MB], 16.6, there exists a finite generically étale map $W \rightarrow\left(\overline{f(C)} \times{ }_{\mathbf{M}} \mathcal{M}\right)_{\text {red. }}$. Then by Lemma 2.3.3, the scheme $\overline{f(C)}$ is the moduli space of $\left(\overline{f(C)} \times_{\mathrm{M}} \mathcal{M}\right)_{\text {red }}$, so, by [L-MB, 11.5 , the stack $\left(\overline{f(C)} \times_{\mathbf{M}} \mathcal{M}\right)_{\text {red }}$ is generically étale over $\overline{f(C)}$. So it follows that $W$ is also generically étale over $\overline{f(C)}$.

Let $F \rightarrow W \times{ }_{\mathbf{M}} C$ be the normalization of an irreducible component of $W \times{ }_{\mathbf{M}} C$ mapping surjectively onto $C$. By refining $T$, we may assume that

(1) $F \rightarrow T$ is smooth;

(2) there exists an open subset $C_{0} \subseteq C$ such that

(a) $C \backslash C_{0}$ is a union of disjoint sections $\Xi$ containing $\Sigma$,

(b) if we denote by $h: F \rightarrow C$, then $F_{0} \stackrel{\text { def }}{=} h^{-1}\left(C_{0}\right) \rightarrow C_{0}$ is étale, and

(c) the scheme of automorphisms of $\beta: F \rightarrow \mathcal{M}$ obtained by composing $F \rightarrow W \rightarrow \mathcal{M}$ is étale over $F_{0}$ of constant degree $\alpha$.

Lemma 7.2.2. There exists a morphism $T_{1} \rightarrow T$ of finite type, and a morphism $E \rightarrow F_{T_{1}}$ such that $E \rightarrow T_{1}$ is smooth of relative dimension $1, E \rightarrow F_{T_{1}}$ is étale over $\left(F_{0}\right)_{T_{1}}$ of degree $\alpha$, and tame over $\left(F \backslash F_{0}\right)_{T_{1}}$, with the following property.

For every geometric point $t: \operatorname{Spec} \Omega \rightarrow T$ and every tame cover $E^{\prime} \rightarrow F_{t}$ of order $\alpha$ which is étale over $\left(F_{0}\right)_{t}$, there exists a lifting $t_{1}: \operatorname{Spec} \Omega \rightarrow T_{1}$, such that $E^{\prime} \rightarrow F_{t}$ is isomorphic to $E_{t_{1}}$ as a covering of $F_{t}$.

Proof. Since $E^{\prime} \rightarrow F_{t}$ is tame of degree $\alpha$, the genus of every connected component of $E^{\prime}$ is bounded by some integer $g_{e}$. It is sufficient to consider one connected component of $E^{\prime}$ at a time; call $\alpha^{\prime}$ its degree over $F_{t}$. Consider the stack of maps $\mathcal{K}_{g_{e}}\left(F / T,\left[\alpha^{\prime}\right]\right)$; there is a locally closed substack $\mathcal{K}_{0}$ consisting of maps which are étale over $F_{0}$ and with smooth source curve. There exists a scheme $T^{\prime} \rightarrow \mathcal{K}_{0}$ surjective and of finite type; let $\bar{E} \rightarrow F_{T^{\prime}}$ be the corresponding universal family. We use the following well-known lemma:

Lemma 7.2.3. There exists a constructible subset $T^{\text {tame }} \subseteq T^{\prime}$ such that for a geometric point $t^{\prime}:$ Spec $\Omega \rightarrow T^{\prime}$ the fiber $\bar{E}_{t^{\prime}} \rightarrow F_{t^{\prime}}$ is a tame covering if and only if $t^{\prime}$ is in $T^{\text {tame }}$.

Proof. By noetherian induction it suffices to show that there is an open set where the lemma holds, and thus we may replace $T^{\prime}$ by a dominant generically finite scheme over it. Let $\bar{\eta}$ be the geometric generic point of $T^{\prime}$, and let $\tilde{E}_{\bar{\eta}} \rightarrow F_{\bar{\eta}}$ be the normalized $\mathfrak{S}_{n}$ cover associated with $E_{\bar{\eta}} \rightarrow F_{\bar{\eta}}$, where $\mathfrak{S}_{n}$ is the symmetric group. There is a finite extension $\eta^{\prime \prime} \rightarrow \eta$ such that the $\mathfrak{S}_{n}$ cover $\tilde{E}_{\bar{\eta}} \rightarrow F_{\bar{\eta}}$ comes 
from $\tilde{E}_{\eta^{\prime \prime}} \rightarrow F_{\eta^{\prime \prime}}$. There is a dominant generically finite $T^{\prime \prime} \rightarrow T^{\prime}$ such that $\eta^{\prime \prime}$ is the generic point of $T^{\prime \prime}$ and $\tilde{E}_{\eta^{\prime \prime}} \rightarrow F_{\eta^{\prime \prime}}$ comes from $\tilde{E}_{T^{\prime \prime}} \rightarrow F_{T^{\prime \prime}}$ and the latter is the normalized $\mathfrak{S}_{n}$ cover associated with $E_{T^{\prime \prime}} \rightarrow F_{T^{\prime \prime}}$. The set of fixed points of elements of order $p$ in $\mathfrak{S}_{n}$ is closed in $\tilde{E}_{T^{\prime \prime}}$, and the image in $T^{\prime}$ is constructible.

To conclude the proof of Lemma 7.2.2, we take $S_{1}$ to be a disjoint union of locally closed subschemes of $T^{\prime}$ covering $T^{\text {tame }}$.

Consider $\widetilde{E}_{2} \stackrel{\text { def }}{=}\left(E \times_{C_{S_{1}}} E\right)^{\text {norm }}, \widetilde{E}_{3} \stackrel{\text { def }}{=}\left(E \times_{C_{S_{1}}} E \times_{C_{S_{1}}} E\right)^{\text {norm }}$. By Abhyankar's Lemma, $\widetilde{E}_{2}, \widetilde{E}_{3} \rightarrow T_{1}$ are smooth.

We have two morphisms $\alpha_{1}, \alpha_{2}: \widetilde{E}_{2} \rightarrow \mathcal{M}$ obtained by composing the map $E \rightarrow$ $F \rightarrow W \rightarrow \mathcal{M}$ with the two projections $\widetilde{E}_{2} \rightarrow E$. Consider the scheme $\widetilde{I}_{2}=$ $\operatorname{Isom}_{\widetilde{E}_{2}}\left(\alpha_{1}, \alpha_{2}\right)$. The morphism $\widetilde{I}_{2} \rightarrow \widetilde{E}_{2}$ is finite, and therefore $\widetilde{I}_{2}$ is projective over $T_{1}$. So there exists a scheme $T_{2}=\operatorname{Sect}_{T_{1}}\left(\widetilde{I}_{2} \rightarrow \widetilde{E}_{2}\right)$, quasiprojective over $T_{1}$, parametrizing sections of $\widetilde{I}_{2} \rightarrow \widetilde{E}_{2}$. In other words, there exists a diagram

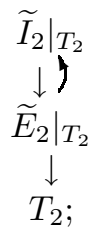

denote the universal section $\sigma:\left.\left.\widetilde{E}_{2}\right|_{T_{2}} \rightarrow \widetilde{I}_{2}\right|_{T_{2}}$.

Considering the three natural projections $p_{12}, p_{13}, p_{23}: \widetilde{E}_{3} \rightarrow \widetilde{E}_{2}$, we have a composition map $\mu: p_{12}^{*} \widetilde{I}_{2} \times p_{23}^{*} \widetilde{I}_{2} \rightarrow p_{13}^{*} \widetilde{I}_{2}$. There exists a closed subscheme $T_{3} \subseteq T_{2}$ where the composition

(*) $\mu \circ\left(p_{12}^{*} \sigma \times p_{23}^{*} \sigma\right): \widetilde{E}_{3} \rightarrow p_{12}^{*} \widetilde{I}_{2} \times p_{23}^{*} \widetilde{I}_{2} \rightarrow p_{13}^{*} \widetilde{I}_{2} \quad$ equals $\quad p_{13}^{*} \sigma: \widetilde{E}_{3} \rightarrow p_{13}^{*} \widetilde{I}_{2}$.

Lemma 7.2.4. There exists a lifting $\lambda:\left(C_{0}\right)_{T_{3}} \rightarrow \mathcal{M}$ of $C \rightarrow \mathbf{M}$, such that the following holds: whenever $t$ : Spec $\Omega \rightarrow T$ is a geometric point and $\tau:\left(C_{0}\right)_{t} \rightarrow \mathcal{M}$ is a lifting of $C_{t} \rightarrow \mathbf{M}$, there exists a lifting $t_{3}: \operatorname{Spec} \Omega \rightarrow T_{3}$ and an isomorphism $t_{3}^{*} \lambda \simeq \tau$.

Proof. The section $\left.\sigma_{3} \stackrel{\text { def }}{=} \sigma\right|_{T_{3}}:\left(\widetilde{E}_{2}\right)_{T_{3}} \rightarrow\left(\widetilde{I}_{2}\right)_{T_{3}}$, when restricted to the open set $E_{0}:=$ the inverse image of $C_{0}$, gives descent data for constructing $\lambda:\left(C_{0}\right)_{T_{3}} \rightarrow \mathcal{M}$, which is effective since $\mathcal{M}$ is a stack. Given a geometric point $t$ : Spec $\Omega \rightarrow T$ and a lifting $\tau:\left(C_{0}\right)_{t} \rightarrow \mathcal{M}$ of $C_{t} \rightarrow \mathbf{M}$, we have a diagram

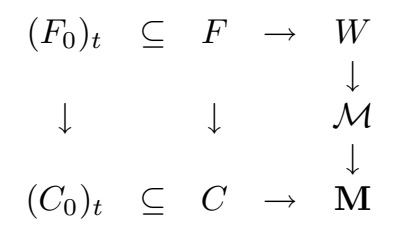

giving two morphisms $\beta, \beta_{2}:\left(F_{0}\right)_{t} \rightarrow \mathcal{M}$ which induce the same morphism $\left(F_{0}\right)_{t} \rightarrow$ M. Therefore the scheme $\operatorname{Isom}_{\left(F_{0}\right)_{t}}\left(\beta, \beta_{2}\right)$ is étale over $F_{0}$, as it is a torsor under $\operatorname{Aut}_{\left(F_{0}\right)_{t}}(\beta)$. For the same reason, it is tame along $\left(F \backslash F_{0}\right)_{t}$. Thus there exists a lifting $t_{1}$ : Spec $\Omega \rightarrow T_{1}$ such that $E_{t_{1}} \rightarrow F_{t_{1}}$ is isomorphic to the normalization of $F_{t_{1}}$ in $\operatorname{Isom}_{\left(F_{0}\right)_{t}}\left(\beta, \beta_{2}\right)$. In particular, we have an isomorphism of $\left.\beta\right|_{E_{T_{1}}}$ with $\beta_{2}$; by conjugation this gives a section $\left.\left(\left(\widetilde{E}_{2}\right)_{t_{1}}\right)\right|_{E_{0}} \rightarrow \widetilde{I}_{2}$, which extends to $\left(\widetilde{E}_{2}\right)_{t_{1}} \rightarrow \widetilde{I}_{2}$, 
since $\left(\widetilde{E}_{2}\right)_{t_{1}}$ is a smooth curve. This extended morphism is easily seen to satisfy the cocycle condition $(*)$, so it gives a point $t_{3}$ : Spec $\Omega \rightarrow T_{3}$, and the corresponding descent data give the lifting $\tau:\left(C_{0}\right)_{t} \rightarrow \mathcal{M}$.

Replacing $T_{3}$ by a regular stratification, this proves the following:

Lemma 7.2.5. There exist a morphism $T_{0} \rightarrow T$ of finite type, with $T_{0}$ regular, and a lifting $\left(C_{0}\right)_{T_{0}} \rightarrow \mathcal{M}$, such that whenever $t: \operatorname{Spec} \Omega \rightarrow T$ is a geometric point and $\tau:\left(C_{0}\right)_{t} \rightarrow \mathcal{M}$ is a lifting of $C_{t} \rightarrow \mathbf{M}$, there exists a lifting $t_{0}:$ Spec $\Omega \rightarrow T_{0}$ and an isomorphism $t_{0}^{*} \lambda \simeq \tau$.

This gives an exhaustive family of generic objects over $C_{0}$. We can now consider extensions of these to twisted objects.

Lemma 7.2.6. Let $C \rightarrow$ Spec $\kappa$ be a smooth curve over an algebraically closed field, $C_{0}=C \backslash\left\{p_{1}, \ldots, p_{n}\right\}$ an open subset, $C_{0} \rightarrow \mathcal{M}$ a morphism. Then there exists a twisted pointed curve $\left(\mathcal{C}, \bar{\Xi}_{1}, \ldots, \bar{\Xi}_{n}\right)$, unique up to a unique isomorphism, with moduli space $\left(C, p_{1}, \ldots, p_{n}\right)$ and a representable morphism $\mathcal{C} \rightarrow \mathcal{M}$ extending $C_{0} \rightarrow \mathcal{M}$.

Proof. Choose an étale neighborhood $U_{i} \rightarrow \mathcal{M}$ of the inverse image in $\mathcal{M}$ of $f\left(p_{i}\right)$, such that $U_{i} \times_{\mathbf{M}} \mathcal{M}=\left[V_{i} / \Gamma_{i}\right]$, where $\Gamma_{i}$ is a finite group. Consider

$$
V_{i} \times{ }_{\mathrm{M}} C \rightarrow U_{i} \times{ }_{\mathrm{M}} C \rightarrow C .
$$

Passing to an étale neighborhood $U_{i}^{\prime} \rightarrow U_{i} \times{ }_{\mathrm{M}} C \rightarrow C$ of $p_{i}$, we may assume that the normalization $V_{i}^{\prime}$ of some irreducible component of $U_{i}^{\prime} \times_{U_{i}} V_{i}$ has exactly one point $q_{i}$ over $p_{i}$. Let $\Gamma_{i}^{\prime}$ be the stabilizer of $V_{i}^{\prime}$. Note that $\Gamma_{i}^{\prime}$ fixes $q_{i}$. Consider the subgroup $\Gamma_{i}^{\prime \prime} \subset \Gamma_{i}^{\prime}$ stabilizing the object $q_{i} \rightarrow \mathcal{M}$. Denote $\bar{V}_{i}^{\prime}=V_{i}^{\prime} / \Gamma_{i}^{\prime \prime}, \bar{\Gamma}_{i}^{\prime}=\Gamma_{i}^{\prime} / \Gamma_{i}^{\prime \prime}$, and $\bar{q}_{i}$ the image of $q_{i}$. Refining $U_{i}^{\prime}$ if necessary, we may assume that $\bar{q}_{i}$ is the unique fixed point of $\bar{\Gamma}_{i}^{\prime}$ in $\bar{V}_{i}^{\prime}$. By Corollary 2.5.2 we have that

$$
\left(\bar{V}_{i}^{\prime} \rightarrow C, \bar{V}_{i}^{\prime} \rightarrow \mathcal{M}, \bar{\Gamma}_{i}^{\prime}\right)
$$

forms a chart at $p_{i}$.

Uniqueness follows from the separatedness of $\mathcal{M}$.

We return to the proof of the proposition. Let $\bar{\eta} \rightarrow T_{0}$ be the geometric generic point. By the lemma, there exists a representable lifting

$$
\mathcal{C}_{\bar{\eta}} \rightarrow \mathcal{M}
$$

over a twisted curve $\left(\mathcal{C}_{\bar{\eta}},\left(\Xi_{1}\right)^{\mathcal{C}_{\bar{\eta}}}, \ldots,\left(\Xi_{n}\right)^{\mathcal{C}_{\bar{\eta}}}\right)$.

There exists a dominant quasifinite morphism $T_{1} \rightarrow T_{0}$ such that the lifting above descends to $T_{1}$. We claim that there exists an open dense subscheme $T_{2} \subset T_{1}$ such that $\mathcal{C}_{T_{2}}$ is representable. This follows from the following well-known fact:

Lemma 7.2.7. Let $f: \mathcal{X}_{1} \rightarrow \mathcal{X}_{2}$ be a morphism of Deligne-Mumford stacks, and let $\mathcal{X} \rightarrow S$ be proper. Then there is an open subset $S_{0} \subset S$ such that if $s: \operatorname{Spec} \Omega \rightarrow S$ is a geometric point, the restricted morphism $f_{s}:\left(\mathcal{X}_{1}\right)_{s} \rightarrow \mathcal{X}_{1}$ is representable if and only if $s$ factors through $S_{0}$.

Proof. Consider the morphism of inertia stacks $\mathcal{I}_{\mathcal{X}_{1}} \rightarrow f^{*} \mathcal{I}_{\mathcal{X}_{2}}$. The kernel is a finite unramified representable group stack $\mathcal{K} \rightarrow \mathcal{X}_{1}$, therefore the complement $\mathcal{K}^{0} \subset \mathcal{K}$ of the identity is also finite over $\mathcal{X}_{1}$. Then $S_{0}$ is the complement of the image of $\mathcal{K}^{0}$ in $S$, which is closed. 
We may now assume that we have a lifting $\mathcal{C} \rightarrow \mathcal{M}$ over $T_{1}$. To conclude the proof of the proposition, there exists an open and closed subscheme $T^{\prime} \subset T_{1}$, such that for any section $\Xi_{i}$ in $\Xi \backslash \Sigma$ the inertia group of a geometric point in $\Xi_{i}^{\mathcal{C}}$ is trivial if and only if the point lies over $T^{\prime}$.

7.3. Proof of Theorem 7.1.1. By taking a surjective map of finite type $T_{1} \rightarrow T$ we may assume that:

(1) $C \rightarrow T$ is of locally constant topological type.

(2) All irreducible components of $C$ have geometrically irreducible fibers.

(3) Any irreducible component $N_{i}$ of the singular locus of $C \rightarrow T$ maps isomorphically to its image.

This implies that the normalization $C^{\text {norm }} \rightarrow C$ can be viewed as a union of families of smooth pointed curves with a stable map to $\mathbf{M}$. By Proposition 7.2 .1 we may assume that there is a dominant morphism $T_{0} \rightarrow T$ of finite type, and a lifting

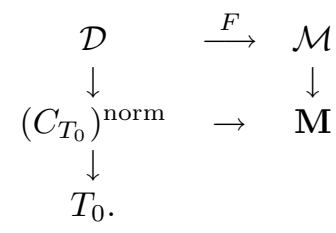

For each $N_{i}$, let $\bar{\Sigma}_{i, 1}, \bar{\Sigma}_{i, 2} \subset C^{\text {norm }}$ be the two sections over $N_{i}$, and $\Sigma_{i, 1}, \Sigma_{i, 2} \subset \mathcal{D}$ the gerbes in $\mathcal{D}$ over them. Replacing $T_{0}$ by an étale cover we may assume that $\Sigma_{i, 1} \rightarrow T_{0}$ and $\Sigma_{i, 2} \rightarrow T_{0}$ have sections $\sigma_{i, 1}: T_{0} \rightarrow \Sigma_{i, 1}$ and $\sigma_{i, 2}: T_{0} \rightarrow \Sigma_{i, 2}$. Refining $T_{0}$ we may assume that there are charts

$$
\left(U_{i, j}, \eta_{i, j}, \Gamma_{i, j}\right)
$$

for $\mathcal{D}$ along $\Sigma_{i, 1}$ and $\Sigma_{i, 2}$ and that the sections $\sigma_{i, j}$ lift to $\widetilde{\sigma}_{i, j}: T_{0} \rightarrow U_{i, j}$. Also we may assume that $\widetilde{\sigma}_{i, j}$ surjects onto the inverse image of $\sigma_{i, j}$ in $U_{i, j}$. This implies that the groups $\Gamma_{i, j}$ are cyclic, and isomorphic to $\operatorname{Aut}\left(\sigma_{i, j}\right)$.

Denote $\tau_{i, j}=F \circ \sigma_{i, j}: T_{0} \rightarrow \Sigma_{i, j} \rightarrow \mathcal{M}$. We have a canonical isomorphism $\tau_{i, j}=\widetilde{\sigma}_{i, j}^{*} \eta_{i, j}$, thus there is an embedding of $\Gamma_{i, j} \times T_{0} \subset \operatorname{Aut}\left(\tau_{i, j}\right)$. Consider the subscheme $T_{1}$ of the scheme of isomorphisms $\operatorname{Isom}_{T_{0}}\left(\tau_{i, 1}, \tau_{i, 2}\right)$ corresponding to isomorphisms which identify $\Gamma_{i, 1}$ with $\Gamma_{i, 2}$. Refining $T_{0}$, we may assume that $T_{1}$ is a union of disjoint copies of $T_{0}$. Consider a section $\psi: T_{0} \rightarrow T_{1}$.

We choose:

(1) a geometric point $t_{0}: \operatorname{Spec} \Omega \rightarrow T_{0}$,

(2) a universal deformation space $W$ for $\mathcal{M}$ at $t_{0}^{*} \tau_{i, 1}$, equivariant under the action of $\operatorname{Aut}\left(t_{0}^{*} \tau_{i, 1}\right)$.

By refining $T_{0}$ and $U_{i, j}$, we may assume that there is a lifting $\nu_{i, 1}: U_{i, 1} \rightarrow W$ of $\eta_{i, 1}$.

Now the chosen isomorphism $\psi$ of $\eta_{i, 1}$ with $\eta_{i, 2}$ and the universal property of the deformation space $W$ imply that, after refining $U_{i, 2}$, there is a lifting $\nu_{i, 2}: U_{i, 2} \rightarrow W$ of $\eta_{i, 2}$ such that the two morphisms $\nu_{i, j} \circ \eta_{i, j}: T_{0} \rightarrow W$ coincide.

We thus obtain a morphism

$$
\eta_{i}: U_{i} \stackrel{\text { def }}{=} U_{i, 1} \underset{T_{0}}{\cup} U_{i, 2} \rightarrow W .
$$


The identification of $\Gamma_{i, 1}$ with $\Gamma_{i, 2}$ given by $\psi$ defines an action of $\Gamma_{i, 1}$ on both $U_{i}$. It acts on $W$ via the embedding $\Gamma_{i, 1} \subset \operatorname{Aut}\left(t_{0}^{*} \tau_{i, 1}\right)$. The morphism $\eta$ is $\Gamma_{i, 1^{-}}$ equivariant, therefore

$$
\left(U_{i}, \eta_{i}, \Gamma_{i, 1}\right)
$$

is a chart for a twisted map $\mathcal{C}_{\psi} \rightarrow \mathcal{M}$.

Taking the union over all $\psi: T_{0} \rightarrow T_{1}$, this defines

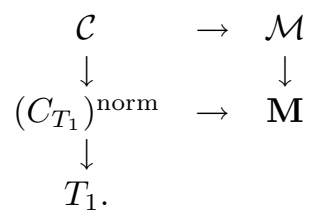

This clearly exhausts all liftings over all geometric points. Setting $T^{\prime}=T_{1}$ completes the proof of Theorem 7.1.1.

7.4. Finiteness of fibers of $\mathbf{K}_{g, n}(\mathcal{M}, d) \rightarrow \mathbf{K}_{g, n}(\mathbf{M}, d)$. To show that $\mathbf{K}_{g, n}(\mathcal{M}, d)$ $\rightarrow \mathbf{K}_{g, n}(\mathbf{M}, d)$ has finite fibers, we need to show that given a stable map $C \rightarrow \mathbf{M}$ over an algebraically closed field, there are only finitely many twisted stable maps $\mathcal{C} \rightarrow \mathcal{M}$ over it. It is easy to see that there are only finitely many possible choices for $\mathcal{C}$, since it admits a finite projective morphism to $C \times_{\mathbf{M}} \mathcal{M}$, in other words, the "twisting" of $\mathcal{C}$ is bounded by the order of stabilizers in $\mathcal{M}$. Thus the following lemma suffices:

Lemma 7.4.1. Let $\mathcal{C}$ be a twisted curve over an algebraically closed field with coarse moduli space $C$. A morphism $C \rightarrow \mathbf{M}$ can come from at most finitely many representable maps $f: \mathcal{C} \rightarrow \mathcal{M}$, up to isomorphism.

Proof. First of all observe that given an object $\xi \in \mathcal{M}\left(C_{\mathrm{sm}}\right)$ there are at most finitely many twisted objects on $\mathcal{C}$ whose restriction to $C_{\mathrm{sm}}$ is isomorphic to $\xi$; so it is enough to prove that any given map $f: T \rightarrow \mathbf{M}$, where $T$ is a smooth curve, comes from at most finitely many objects on $T$.

Over $\mathbb{C}$, it is easy to deduce this finiteness result analytically: given a base point $t \in T$ and a fixed lifting $\tilde{f}: T \rightarrow \mathcal{M}$, it is easy to associate, in a one-to-one manner, to any lifting $\tilde{f}^{\prime}: T \rightarrow \mathcal{M}$ a monodromy representation $\phi_{f^{\prime}}: \pi_{1}(T) \rightarrow$ Aut $\xi_{t}$. Since $\pi_{1}(T)$ is finitely generated there are only finitely many such representations.

We now give a short algebraic argument using the language of stacks.

Call $\mathcal{T}$ the normalization of the pullback of $\mathcal{M}$ to $T$; if there is at least one map $T \rightarrow \mathcal{M}$ giving rise to $f$, then this induces a section $s: T \rightarrow \mathcal{T}$. It is proved in $\mathrm{Vi}$ that this section is étale; the argument is similar to that of the purity lemma: by passing to the strict henselization one can lift $s$ to the universal deformation space. The image of this lifting is then evidently étale, and therefore $s$ is étale. This strongly restricts the structure of $T$.

Call $R$ the fiber product $T \times_{\mathcal{T}} T$; then the étale finite groupoid $R \Longrightarrow T$ is a presentation of $\mathcal{T}$. Each lifting $T \rightarrow \mathcal{M}$ of $f$ comes from a section $T \rightarrow \mathcal{T}$, so it is enough to show that up to isomorphisms there are only finitely many such sections. But such a section $\sigma: T \rightarrow \mathcal{T}$ is determined by the pullback $U$ of $T \stackrel{s}{\rightarrow} \mathcal{T}$ to $T$ via $\sigma$, and a morphism of groupoids from $U \times_{T} U \Longrightarrow U$ to $R \Longrightarrow T$ over $T$. But up to isomorphism there are only finitely many étale coverings $U \rightarrow T$ of bounded degree, because the tame fundamental group of $T$ is topologically finitely generated. For each $U \rightarrow T$ there are only finitely many liftings $U \times_{T} U \rightarrow R$. 
7.5. Conclusion of the proof of the main theorem. Theorem 7.1 .1 and the fact that the stack $\mathcal{K}_{g, n}(\mathbf{M}, d)$ is of finite type (see, e.g., [B-M]) imply that the stack $\mathcal{K}_{g, n}(\mathcal{M}, d)$ is of finite type. Since the stack has finite diagonal and satisfies the weak valuative criterion for properness, it is proper. The induced morphism $\mathbf{K}_{g, n}(\mathcal{M}, d) \rightarrow \mathbf{K}_{g, n}(\mathbf{M}, d)$ of coarse moduli spaces is proper with finite fibers. Since $\mathbf{K}_{g, n}(\mathbf{M}, d)$ is projective, we have that $\mathbf{K}_{g, n}(\mathcal{M}, d)$ is projective as well. We have already seen that $\mathcal{K}_{g, n}(\mathcal{M}, d) \rightarrow \mathcal{K}_{g, n}(\mathbf{M}, d)$ is of Deligne-Mumford type and tame.

\section{Some Properties AND Generalizations}

\subsection{Balanced maps.}

Proposition 8.1.1. The stack $\mathcal{K}_{g, n}^{\mathrm{bal}}(\mathcal{M}, d)$ of balanced twisted stable maps is an open and closed substack in $\mathcal{K}_{g, n}(\mathcal{M}, d)$.

This is immediate from Lemma 5.4 .3

8.2. Fixing classes in Chow groups modulo algebraic equivalence. Assume $\mathbb{S}$ is the spectrum of a field. Given a class $\beta$ in the Chow group of cycles of dimension 1 on $\mathbf{M}$ modulo algebraic equivalence, such that its intersection number with the Chern class of the chosen ample sheaf is $d$, we have an open and closed substack $\mathcal{K}_{g, n}(\mathbf{M}, \beta) \subset \mathcal{K}_{g, n}(\mathbf{M}, d)$. Pulling back, we have an open and closed substack $\mathcal{K}_{g, n}(\mathcal{M}, \beta) \subset \mathcal{K}_{g, n}(\mathcal{M}, d)$. A similar construction can be obtained using classes on $\mathcal{M}$, whenever one can construct them with integral coefficients; see, e.g., [E-G], $\underline{\mathrm{Kr}}$.

8.3. Stable maps over a base stack. Suppose we are given a tame morphism $\mathcal{M} \rightarrow \mathcal{S}$ of algebraic stacks, where $\mathcal{S}$ is noetherian. Suppose further that it factors as $\mathcal{M} \rightarrow \mathbf{M} \rightarrow \mathcal{S}$, where $\mathcal{M} \rightarrow \mathbf{M}$ is proper and quasifinite, and $\mathbf{M} \rightarrow \mathcal{S}$ is projective (but we do not assume that $\mathbf{M}$ is representable). We can define a category $\mathcal{K}_{g, n}(\mathcal{M} / \mathcal{S}, d)$ of twisted stable maps exactly as before. We claim that the analogue of the main theorem still holds, which may be of use in some applications.

The point is the following: most arguments in this paper go through word for word. To produce a smooth parametrization take a smooth surjective morphism $\mathbb{S} \rightarrow \mathcal{S}$, and denote by $\mathcal{M}_{1}$ the pullback of $\mathcal{M}$ to $\mathbb{S}$. Then

$$
\mathcal{K}_{g, n}\left(\mathcal{M}_{1} / \mathbb{S}, d\right) \rightarrow \mathcal{K}_{g, n}(\mathcal{M} / \mathcal{S}, d)
$$

is representable, smooth, surjective and of finite type. Composing with a smooth parametrization of the stack $\mathcal{K}_{g, n}\left(\mathcal{M}_{1} / \mathbb{S}, d\right)$ we get what we want. To show that $\mathcal{K}_{g, n}(\mathcal{M} / \mathcal{S}, d) \rightarrow \mathcal{S}$ is proper, note that its pullback after a faithfully flat base change to a scheme is proper.

8.4. When $\mathbf{M}$ is only a proper algebraic space. So far we have assumed that $\mathbf{M} \rightarrow \mathbb{S}$ is projective. In case $\mathbf{M} \rightarrow \mathbb{S}$ is only proper, one can proceed as follows.

First, it was pointed out by Johan de Jong that in this case $\mathcal{K}_{g, n}(\mathbf{M}, \beta)$ is an algebraic stack, locally of finite type, such that each irreducible component is proper over $\mathbb{S}$. The only part which is not evident is the properness of the irreducible components. This can be seen as follows: let $\eta$ be a generic point of such an irreducible component, with a stable map $\left(C_{\eta} \rightarrow\{\eta\}, \Sigma_{i}, C_{\eta} \rightarrow \mathbf{M}\right)$. An easy gluing argument allows us to reduce to the case where $C_{\eta}$ is irreducible. We then replace $\mathbf{M}$ by the closure of the image of $C_{\eta}$. There is a birational morphism 
$\mathbf{M}^{\prime} \rightarrow \mathbf{M}$ such that $\mathbf{M}^{\prime} \rightarrow \mathbb{S}$ is projective. There is a lifting $C_{\eta} \rightarrow \mathbf{M}^{\prime}$, which has a certain degree $d$ with respect to some ample sheaf on $\mathbf{M}^{\prime}$. The map $C \rightarrow \mathbf{M}^{\prime}$ induces $\eta \rightarrow \mathcal{K}_{g, n}\left(\mathbf{M}^{\prime}, d\right)$. Take the closure $\mathcal{K}$ of the image, which is a proper stack. There is a universal stable map $\left(C^{\prime} \rightarrow \mathcal{K}, \Sigma_{i}^{\prime}, C^{\prime} \rightarrow \mathbf{M}^{\prime}\right)$. The composite map $\left(C^{\prime} \rightarrow \mathcal{K}, \Sigma_{i}^{\prime}, C^{\prime} \rightarrow \mathbf{M}^{\prime} \rightarrow \mathbf{M}\right)$ may be unstable, but using Knudsen's contraction procedure we obtain an associated map $\left(C \rightarrow \mathcal{K}, \Sigma_{i}, C \rightarrow \mathbf{M}\right)$, giving a morphism $\mathcal{K} \rightarrow \mathcal{K}_{g, n}(\mathbf{M}, \beta)$ whose image, which is the component with which we started, is proper.

Now considering a tame stack $\mathcal{M}$ proper over $\mathbb{S}$, the arguments of the main theorem along with the one above show that $\mathcal{K}_{g, n}(\mathcal{M}, \beta)$ is again an algebraic stack, locally of finite type, such that each irreducible component is proper over $\mathbb{S}$.

It can be shown that a similar result holds when $\mathcal{M}$ is only tame, separated and of finite type, without properness. The crucial point is that $\mathcal{K}_{g, n}(\mathbf{M})$ exists in such a situation (though is not proper, not even of finite type), and then one can apply our arguments, or simply apply our main theorem working with schemes over $\mathbf{K}_{g, n}(\mathbf{M})$ rather than over $\mathbb{S}$, with target stack $C_{\text {Univ }} \times{ }_{\mathbf{M}} \mathcal{M}$ with $C_{\text {Univ }} \rightarrow \mathcal{K}_{g, n}(\mathbf{M})$ the universal curve, rather than $\mathcal{M}$. Unfortunately we are not aware of a treatment of $\mathcal{K}_{g, n}(\mathbf{M})$ in this general case in the literature. A particular open and closed substack of $\mathcal{K}_{g, n}(\mathcal{M})$ whose existence is clear is $\mathcal{K}_{g, n}(\mathcal{M} / \mathbf{M}, 0)$, the stack of maps which are constant on moduli spaces. In this case $\mathcal{K}_{g, n}(\mathbf{M} / \mathbf{M}, 0) \simeq \mathbf{M} \times \overline{\mathcal{M}}_{g, n}$. This case is considered in $\$-\mathrm{G}-\mathrm{V}$ ].

8.5. Other open and closed loci. Consider a twisted object $(\xi, C \rightarrow S, \mathcal{A})$, and take a geometric point $c_{0}$ of the support $\Sigma_{i}^{C}$ of the $i$-th section $S \rightarrow C$. If $(U, \eta, \Gamma)$ is a chart in the atlas, and $u_{0}$ is a geometric point of $U$ lying over $c_{0}$, the stabilizer of $u_{0}$ in $\Gamma$ has an order which is independent of the chart. We call this order the local index of the twisted object at $c_{0}$; we can think of it as a measure of how twisted the object is around $c_{0}$. One immediately checks that the local index only depends on the image of $c_{0}$ in $S$; this way we get a function $\epsilon_{i}: S \rightarrow \mathbf{N}$.

Proposition 8.5.1. The function $\epsilon_{i}: S \rightarrow \mathbf{N}$ is locally constant.

Proof. Let $c_{0}$ be a geometric point of $\Sigma_{i}^{C},(U, \eta, \Gamma)$ a chart, $u_{0}$ a geometric point of $U$ lying over $c_{0}$. By refining the chart we may assume that $u_{0}$ is a fixed point of $\Gamma$. Since the morphism $\Sigma_{i}^{C} \rightarrow S$ is étale, it follows that $\Gamma$ leaves the whole component of $\Sigma_{i}^{C}$ containing $u_{0}$ fixed, and the thesis follows easily from this.

Definition 8.5.2. Let $\mathbf{e}=\left(e_{1}, \ldots, e_{n}\right)$ be a sequence of positive integers. A twisted object has global index equal to $\mathbf{e}$ if the value of the associated function $\epsilon_{i}$ on $S$ is constant equal to $e_{i}$ for all $i=1, \ldots, n$.

Sometimes the local index can be further refined. For example, if $G$ is a finite group, and $\mathcal{B} G$ is the classifying stack of $G$ over $\mathbb{S}$, that is, the stack whose objects are Galois étale covers with group $G$, then the stabilizer of a geometric point of $\Sigma_{i}^{U}$ is a cyclic subgroup of the automorphism group of an étale Galois cover of the spectrum of an algebraically closed field. This group is isomorphic to $G$, and the isomorphism is well defined up to conjugation, so from each geometric point of $\Sigma_{i}^{U}$ one gets a conjugacy class of cyclic subgroups of $G$, which only depends on the index $i$ and on the image of the geometric point in $S$. It is easy to check that this conjugacy class is locally constant on $S$. 


\section{FUNCTORIALITY OF THE STACK OF TWISTED STABLE MAPS}

9.1. Statements. Let $\mathcal{C} \rightarrow T$ be a proper $n$-pointed twisted curve of genus $g$ and let $\mathcal{C} \rightarrow \mathcal{M}$ be a morphism of stacks. Consider the corresponding morphism of coarse moduli spaces $C \rightarrow \mathbf{M}$. Assume that either $C \rightarrow \mathbf{M}$ is nonconstant or $2 g-2+n>0$. It is well known (see [B-M], Proposition 3.10) that $C \rightarrow \mathbf{M}$ is stabilizable: there exists a canonical proper surjective morphism $C \rightarrow C^{\prime}$ of pointed curves which gives a factorization $C \rightarrow C^{\prime} \rightarrow \mathbf{M}$ such that $C^{\prime} \rightarrow M$ is a stable pointed map and the fibers $C \rightarrow C^{\prime}$ are the nonstable trees of rational curves.

Proposition 9.1.1. There exists a factorization $\mathcal{C} \rightarrow \mathcal{C}^{\prime} \rightarrow \mathcal{M}$ such that

(1) $\mathcal{C}^{\prime} \rightarrow \mathcal{M}$ is a twisted stable map, and

(2) on the level of coarse moduli spaces this induces the factorization $C \rightarrow C^{\prime} \rightarrow$ M.

The factorization is unique up to a unique isomorphism. The formation of $\mathcal{C}^{\prime}$ commutes with base change on $T$. Moreover, if $\mathcal{C} \rightarrow T$ is balanced, then so is $\mathcal{C}^{\prime}$.

We denote $\mathcal{K}_{g, n}(\mathcal{M}, *)=\bigcup_{d} \mathcal{K}_{g, n}(\mathcal{M}, d)$. The following corollaries follow immediately from the proposition:

Corollary 9.1.2. Let $\phi: \mathcal{M} \rightarrow \mathcal{M}^{\prime}$ be a morphism of proper tame stacks over $\mathbb{S}$ having projective coarse moduli spaces. Denote by $\mathcal{K}_{g, n}^{[\phi]}(\mathcal{M}, d) \subset \mathcal{K}_{g, n}(\mathcal{M}, d)$ the open and closed locus where $C \rightarrow \mathbf{M}^{\prime}$ is stabilizable: if $2 g-2+n>0$, then $\mathcal{K}_{g, n}^{[\phi]}(\mathcal{M}, d)=\mathcal{K}_{g, n}(\mathcal{M}, d)$. Otherwise it is the locus where the composite map of moduli spaces $C \rightarrow \mathbf{M} \rightarrow \mathbf{M}^{\prime}$ is nonconstant. Then there exists a morphism $\mathcal{K}_{g, n}^{[\phi]}(\mathcal{M}, *) \rightarrow \mathcal{K}_{g, n}\left(\mathcal{M}^{\prime}, *\right)$, sending balanced maps to balanced maps, which makes the following diagram commutative:

$$
\begin{array}{ccc}
\mathcal{K}_{g, n}^{[\phi]}(\mathcal{M}, *) & \rightarrow & \mathcal{K}_{g, n}\left(\mathcal{M}^{\prime}, *\right) \\
\downarrow & & \downarrow \\
\mathcal{K}_{g, n}^{[\phi]}(\mathbf{M}, *) & \rightarrow & \mathcal{K}_{g, n}\left(\mathbf{M}^{\prime}, *\right)
\end{array}
$$

where the vertical arrows are the canonical maps described in Theorem 1.4.1 and the morphism $\mathcal{K}_{g, n}^{[\phi]}(\mathbf{M}, *) \rightarrow \mathcal{K}_{g, n}\left(\mathbf{M}^{\prime}, *\right)$ is the one described by Knudsen and BehrendManin, induced by the contraction of rational trees which become nonstable over $\mathbf{M}^{\prime}$.

Corollary 9.1.3. Let $\mathcal{K}_{g, n}^{\prime}(\mathcal{M}, d) \subset \mathcal{K}_{g, n}(\mathcal{M}, d)$ be the open and closed substack where the last marking $\Sigma_{n}$ is representable. Assume either $d>0$ or $2 g-2+n>1$. Then there exists a morphism $\mathcal{K}_{g, n}^{\prime}(\mathcal{M}, d) \rightarrow \mathcal{K}_{g, n-1}(\mathcal{M}, d)$, sending balanced maps to balanced maps, which makes the following diagram commutative:

$$
\begin{array}{cccc}
\mathcal{K}_{g, n}^{\prime}(\mathcal{M}, d) & \rightarrow & \mathcal{K}_{g, n-1}(\mathcal{M}, d) \\
\downarrow & & \downarrow \\
\mathcal{K}_{g, n}^{\prime}(\mathbf{M}, d) & \rightarrow & \mathcal{K}_{g, n-1}(\mathbf{M}, d)
\end{array}
$$

where the vertical arrows are the canonical maps described in Theorem 1.4.1 and the morphism $\mathcal{K}_{g, n}^{\prime}(\mathbf{M}, d) \rightarrow \mathcal{K}_{g, n-1}(\mathbf{M}, d)$ is the one described by Knudsen and Behrend-Manin, induced by the contraction of rational components which become nonstable when forgetting the last marking $\Sigma_{n}^{C}$.

Remark 9.1.4. Given a $T$-scheme of finite type $U \rightarrow T$, not necessarily proper, one defines a stable divisorially $n$-marked map $\left(C \rightarrow U, \Sigma_{1}, \ldots, \Sigma_{n}\right)$ over $T$ to be a nodal divisorially $n$-marked curve $C \rightarrow T$ and a proper morphism $f: C \rightarrow U$ such 
that, for each geometric point $p$ of $T$, the group $\operatorname{Aut}_{U}\left(f: C_{p} \rightarrow U, \Sigma_{1, p}, \ldots, \Sigma_{n, p}\right)$ is finite. Ideally, one should prove the existence of the contraction of nonstable trees $C \rightarrow C^{\prime} \rightarrow U$ for divisorially $n$-marked maps $\left(C \rightarrow U, \Sigma_{1}, \ldots, \Sigma_{n}\right)$ even in the nonproper case, and then deduce our proposition using a presentation $R \Longrightarrow U$ of $\mathcal{M}$. There are some technical difficulties in carrying out such a local construction. We will use the uniqueness of the local construction to deduce the uniqueness in the twisted case, but we will use a global approach for proving existence.

9.2. Twisted nonstable trees. Before proving the proposition, we state two lemmas which illuminate the geometric picture underlying the proposition. Consider a twisted curve $\mathcal{E}$ over an algebraically closed field, and a representable morphism $\mathcal{E} \rightarrow \mathcal{M}$ which is constant on moduli spaces. If $p$ is the image point in $\mathbf{M}$, then the reduction of its inverse image in $\mathcal{M}$ is the classifying stack $\mathcal{B} G$ of the automorphism group of a geometric point of $\mathcal{M}$ lying over $p$. Thus the map $\mathcal{E} \rightarrow \mathcal{M}$ factors through $\mathcal{B} G$.

Lemma 9.2.1. Let $E$ be a tree of rational curves over an algebraically closed field $k$. Let $p \in E$ be a smooth closed point. Let $(\mathcal{E}, \Sigma)$ be a 1-pointed twisted curve with associated coarse curve $(E, p)$. Let $G$ be a finite group with order prime to char $k$, and let $\mathcal{E} \rightarrow \mathcal{B} G$ be a representable morphism. Then $\mathcal{E} \rightarrow E$ is an isomorphism, and the map $\mathcal{E} \rightarrow \mathcal{B} G$ corresponds to the trivial principal bundle.

Proof. We proceed by induction on the number of components of $E$, starting from the case where $E$ is empty, when the statement is vacuous. Let $D \subset E$ be a tail component; in case $E$ is reducible we may assume $p \notin D$, so in any case $D$ contains at most one special point $q$. Denote $D_{0}:=D \backslash\{q\}$.

Let $P \rightarrow \mathcal{E}$ be the principal $G$-bundle corresponding to $\mathcal{E} \rightarrow \mathcal{B} G$. Now restricting $P \rightarrow E$ to $D_{0}$, we obtain a tame principal bundle $P_{D_{0}} \rightarrow D_{0}$, which is trivial, since $\pi_{1}^{\text {tame }}\left(D_{0}\right)=\{1\}$.

Define $\mathcal{D}$ to be the reduction of the inverse image of $D$. The principal bundle $P_{\mathcal{D}} \rightarrow \mathcal{D}$ has a section outside $q$, which automatically extends to $\mathcal{D}$. Therefore $P_{\mathcal{D}} \rightarrow \mathcal{D}$ is trivial, which means that $\mathcal{D} \rightarrow \mathcal{B} G$ factors through Spec $k \rightarrow \mathcal{B} G$. Since Spec $k$ is representable, we have that $\mathcal{D}$ is representable.

By induction, the lemma holds over $\overline{E \backslash D}$. It follows that $\mathcal{E}$ is representable, and since $\pi_{1}(E)=\{1\}$, the principal bundle $P \rightarrow \mathcal{E}$ is trivial.

Lemma 9.2.2. Let $E$ be a tree of rational curves over an algebraically closed field $k$. Let $p_{1}, p_{2} \in E$ be two distinct smooth closed points. Let $\left(\mathcal{E}, \Sigma_{1}, \Sigma_{2}\right)$ be a 2-pointed twisted curve with associated coarse curve $\left(E, p_{1}, p_{2}\right)$. Let $G$ be a finite group with order prime to char $k$, and let $\mathcal{E} \rightarrow \mathcal{B} G$ be a representable morphism. Denote by $F \subset E$ the unique chain of components connecting $p_{1}$ with $p_{2}$. Then:

(1) $\mathcal{E} \rightarrow E$ is an isomorphism away from the special points of $F$, namely $p_{1}, p_{2}$ and the nodes of $F$.

(2) There exists a cyclic subgroup $\Gamma \subset G$ such that the image of the automorphism group of a geometric point $\operatorname{Spec} k \rightarrow E$ lying over any of the special points of $F$ maps isomorphically to $\Gamma$.

(3) Let $P \rightarrow \mathcal{E}$ be the principal $G$-bundle associated to $\mathcal{E} \rightarrow \mathcal{B} G$. Then each connected component of $P$ is a tree of rational curves.

(4) For a geometric point $q \rightarrow \mathcal{E}$ lying over a special point of $F$, we let $\Gamma$ act on the tangent space of $\mathcal{E}$ at $q$ via the isomorphism in (2) above. Assuming $\mathcal{E}$ 
is balanced, then the character of the action of $\Gamma$ on the tangent space to $\mathcal{E}$ at $p_{1}$ is opposite to the character of the action at $p_{2}$.

Proof. Consider a connected component $E^{\prime} \subset \overline{E \backslash F}$. Then $E^{\prime}$ is a tree of rational curves which is attached to $F$ at a unique point $p^{\prime}$. Let $\mathcal{E}^{\prime} \subset \mathcal{E}$ be the reduction of the inverse image of $E^{\prime}$ and let $\Sigma^{\prime}$ be the reduction of the inverse image of $p^{\prime}$ in $\mathcal{E}^{\prime}$. Then we can apply the previous lemma to $\mathcal{E} \rightarrow \mathcal{B} G$ and conclude that $\mathcal{E}^{\prime} \rightarrow E^{\prime}$ is an isomorphism. Thus we may replace $E$ by $F$ and assume that $E=F$.

Let $P \rightarrow \mathcal{E}$ be the principal $G$-bundle associated with the morphism $\mathcal{E} \rightarrow \mathcal{B} G$. Then $P \rightarrow E$ is a tamely ramified covering which is étale outside of the special points. Let $Q \subset P$ be a connected component and let $\Gamma$ be its stabilizer. Note that $\mathcal{E}=[P / G]$, and since the action of $G$ on the set of connected components of $P$ is transitive, we have that $[Q / \Gamma] \rightarrow \mathcal{E}$ is an isomorphism. Let $Q_{1} \subset Q$ be an irreducible component, and $E_{1} \subset E$ its image. Then, since the tame fundamental group of $\mathbb{P}^{1} \backslash\{0, \infty\}$ is cyclic, we know that $Q_{1}$ is a smooth rational curve, and the map $Q_{1} \rightarrow E_{1}$ is totally ramified at exactly the two special points of $E$ on $E_{1}$. This implies that the map of dual graphs $\operatorname{Graph}(Q) \rightarrow \operatorname{Graph}(E)$ is étale, and since $\operatorname{Graph}(E)$ is simply connected this implies that this map of dual graphs is an isomorphism. From this it follows that there is exactly one irreducible component of $Q$ over each irreducible component of $E$ and the results are clear.

9.3. Proof of the proposition. We may assume that $T$ is noetherian, since $\mathcal{C} \rightarrow$ $T$ and $\mathcal{M} \rightarrow T$ are of finite presentation.

Step 1: Reduction to the RePresentable CASE. First we reduce to the case where $\mathcal{C} \rightarrow \mathcal{M}$ is representable, as follows: let $R \Longrightarrow U$ be a presentation of $\mathcal{M}$. Consider the pullbacks $\mathcal{C}_{R} \rightrightarrows \mathcal{C}_{U}$ of $\mathcal{C}$ to $R$ and $U$, respectively. These are divisorially $n$-marked twisted curves. Let $\mathcal{C}_{R} \rightarrow C_{R}$ and $\mathcal{C}_{U} \rightarrow C_{U}$ be the respective coarse moduli spaces, which are divisorially $n$-marked curves. By Lemma2.2.2 there is an induced étale groupoid $C_{R} \Longrightarrow C_{U}$ whose quotient is a twisted pointed curve $\overline{\mathcal{C}} \rightarrow T$ with a representable morphism $\overline{\mathcal{C}} \rightarrow \mathcal{M}$. The coarse moduli space of $\overline{\mathcal{C}}$ is canonically isomorphic to $C$. Note that, since $\mathcal{C} \rightarrow T$ is tame, the formation of $C_{R} \rightrightarrows C_{U}$ commutes with base change on $T$, so the formation of $\overline{\mathcal{C}} \rightarrow T$ also commutes with base change on $T$.

Let us assume that $\mathcal{C} \rightarrow T$ is balanced. In order to show that $\overline{\mathcal{C}} \rightarrow T$ is balanced as well, it is enough to consider the case where $T$ is the spectrum of an algebraically closed field. Let $(V, \Gamma)$ be a chart for $\mathcal{C}$. Without loss of generality we may assume $\Gamma$ is cyclic and has a unique fixed geometric point $q \in V$, and the action of $\Gamma$ is free outside $q$. The morphism $\mathcal{C} \rightarrow \mathcal{M}$ induces a morphism $\Gamma \rightarrow \operatorname{Aut}(q \rightarrow \mathcal{M})$. Denote the kernel of this morphism by $H$ and the image by $\bar{\Gamma}$. Then $(V / H, \bar{\Gamma})$ is a chart for $\overline{\mathcal{C}}$, which is evidently balanced.

Thus from here on we will assume $\mathcal{C} \rightarrow \mathcal{M}$ is representable.

SteP 2: Uniqueness. Let $\mathcal{C} \rightarrow \mathcal{C}^{\prime} \rightarrow \mathcal{M}$ be a contraction as in the proposition. Consider a presentation $R \Longrightarrow U$ of $\mathcal{M}$ and consider the following fiber diagram:

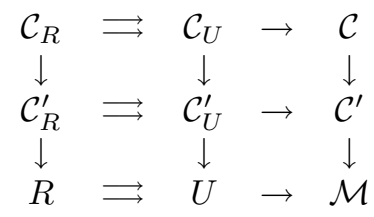


Note that $\mathcal{C}_{R}, \mathcal{C}_{U}, \mathcal{C}_{R}^{\prime}$, and $\mathcal{C}_{U}^{\prime}$ are representable. Moreover, it follows from Lemmas 9.2.1 and 9.2.2 that the fibers of $\mathcal{C}_{U} \rightarrow \mathcal{C}_{U}^{\prime}$ and $\mathcal{C}_{R} \rightarrow \mathcal{C}_{R}^{\prime}$ are precisely the nonstable trees in $\mathcal{C}_{U}$ and $\mathcal{C}_{R}$.

Lemma 9.3.1 below implies that the groupoid $\mathcal{C}_{R}^{\prime} \rightrightarrows \mathcal{C}_{U}^{\prime}$ is uniquely determined as a local contraction of nonstable trees in $\mathcal{C}_{R} \rightrightarrows \mathcal{C}_{U}$, and its formation commutes with base change on $T$. Therefore $\mathcal{C} \rightarrow \mathcal{C}^{\prime} \rightarrow \mathcal{M}$ is unique, and its formation also commutes with base change on $T$.

Lemma 9.3.1. Let $U \rightarrow T$ be a scheme of finite type, $C \rightarrow T$ a divisorially marked curve, and $C \rightarrow U$ a proper morphism. If $C \rightarrow C^{\prime} \rightarrow U$ is a contraction of the nonstable trees, then it is unique up to a canonical isomorphism and its formation commutes with base change.

Proof of the lemma. Note that the last statement follows from unicity. Now $C^{\prime}$ is uniquely determined as a topological space, since the fibers are uniquely determined and the topology is the quotient topology since the map $\pi: C \rightarrow C^{\prime}$ is proper. Therefore it is enough to check that the morphism $\mathcal{O}_{C^{\prime}} \rightarrow \pi_{*} \mathcal{O}_{C}$ is an isomorphism.

Note that this morphism is injective since the kernel is supported in the locus of special points of $C^{\prime} \rightarrow T$, which contains none of the associated points of $C^{\prime}$.

Denote by $Q$ the quotient sheaf

$$
0 \rightarrow \mathcal{O}_{C^{\prime}} \rightarrow \pi_{*} \mathcal{O}_{C} \rightarrow Q \rightarrow 0
$$

We need to show $Q=0$. We may assume that $T$ is the spectrum of a local ring $R$.

(1) Consider the case where $R$ is a field. We may pass to the algebraic closure, and then the statement follows from the fact that nodal curves are seminormal.

(2) Consider the case depth $R>0$. In this case the ideal of the support of $Q$ has depth $>1$. This implies that the sequence $0 \rightarrow \mathcal{O}_{C^{\prime}} \rightarrow \pi_{*} \mathcal{O}_{C} \rightarrow Q \rightarrow 0$ splits locally on $C^{\prime}$, by the $\mathcal{E} x t$ characterization of depth (see $\mathrm{Ma}$, Theorem 16.7). Let $\pi_{*} \mathcal{O}_{C} \rightarrow \mathcal{O}_{C^{\prime}}$ be a local splitting. It is enough to prove that this is injective. Let $f$ be a nonzero section of the kernel of this splitting. By restriction to the central fiber we see that $f \in \mathfrak{m} \mathcal{O}_{C}$, where $\mathfrak{m}$ is the maximal ideal of $R$. Let $k$ be the maximal integer such that $f \in \mathfrak{m}^{k} \mathcal{O}_{C}$. We note that as a section of $\mathfrak{m}^{k} \mathcal{O}_{C}$, the element $f$ vanishes outside the nonstable trees. Since $\left(\mathfrak{m}^{k} / \mathfrak{m}^{k+1}\right) \mathcal{O}_{C}$ is a constant vector bundle on $C_{0}$, we have that the restriction of $f \in \mathfrak{m}^{k} \mathcal{O}_{C}$ to the central fiber is again zero, therefore $f \in \mathfrak{m}^{k+1} \mathcal{O}_{C}$, contradicting the maximality of $k$.

(3) In the general case, denote by $I \subset \mathfrak{m}$ the largest proper ideal of finite length (so $R / I$ is either a field or of positive depth). Let $J \subset I$ be an ideal of length 1. By induction on the length of $I$, we may assume the statement holds true over $\tilde{R}:=R / J$.

The exact sequence

$$
0 \rightarrow J \rightarrow R \rightarrow \tilde{R} \rightarrow 0
$$

induces an exact sequence

$$
0 \rightarrow \mathcal{O}_{C} \otimes J \rightarrow \mathcal{O}_{C} \rightarrow \mathcal{O}_{\tilde{C}} \rightarrow 0 .
$$


Note that $\mathcal{O}_{C} \otimes J$ is isomorphic to $\mathcal{O}_{C_{0}}$, where $C_{0}$ is the central fiber. Applying $\pi_{*}$ we get an exact sequence

$$
0 \rightarrow \pi_{*} \mathcal{O}_{C_{0}} \rightarrow \pi_{*} \mathcal{O}_{C} \rightarrow \pi_{*} \mathcal{O}_{\tilde{C}} \rightarrow R^{1} \pi_{*} \mathcal{O}_{C_{0}} .
$$

Note that the last term is zero. This follows by considering the inverse image of an affine neighborhood of a point on $C^{\prime}$ containing the image of a contracted tree $D \subset C_{0}$ and computing the cohomology long exact sequence of

$$
0 \rightarrow \mathcal{O}_{\overline{C_{0} \backslash D}}(-\Delta) \rightarrow \mathcal{O}_{C_{0}} \rightarrow \mathcal{O}_{D} \rightarrow 0 .
$$

Now we get a commutative diagram

$$
\begin{aligned}
& 0 \rightarrow \mathcal{O}_{C_{0}^{\prime}} \rightarrow \mathcal{O}_{C^{\prime}} \rightarrow \mathcal{O}_{\tilde{C}^{\prime}} \rightarrow 0 \\
& 0 \rightarrow \underset{\mathcal{O}_{C_{0}}}{\downarrow} \rightarrow \stackrel{\mathcal{O}_{C}}{\downarrow} \rightarrow \begin{array}{c}
\downarrow \\
\mathcal{O}_{\tilde{C}}
\end{array} \rightarrow 0
\end{aligned}
$$

where the left and right columns are isomorphisms. This implies that the center column is also an isomorphism, which concludes the proof the lemma.

Step 3: Construction of $\mathcal{C}^{\prime}$. Consider the diagram

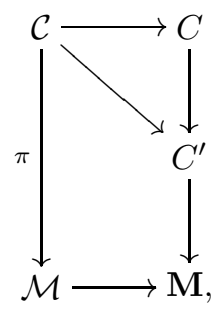

where $C \rightarrow C^{\prime} \rightarrow \mathrm{M}$ is the contraction constructed by Behrend-Manin. Let $L^{\prime}$ be a sufficiently relatively ample invertible sheaf on $C^{\prime} / \mathbf{M}$, and let $\mathcal{L}$ be its pullback to $\mathcal{C}$. Here "sufficiently ample" means that the degrees of $L^{\prime}$ on the components of the fibers of $C^{\prime} \rightarrow \mathbf{M}$ are larger than a certain integer $N$ to be specified later.

We define a quasicoherent sheaf of graded algebras

$$
\mathcal{A}=\bigoplus_{r \geq 0} \pi_{*} \mathcal{L}^{\otimes r}
$$

Claim. (1) For any quasicoherent sheaf of $\mathcal{O}_{T}$-modules $\mathcal{F}$, we have

$$
R^{1} \pi_{*}\left(\mathcal{L}^{\otimes r} \otimes \mathcal{F}\right)=0
$$

for all $r>0$.

(2) For all $r>0$, the formation of $\pi_{*} \mathcal{L}^{\otimes r}$ commutes with base change on $T$, and the sheaf is flat over $T$.

(3) The algebra $\mathcal{A}$ is generated over $\mathcal{O}_{\mathcal{M}}$ in degree 1; in particular it is locally finitely generated.

Proof. Note that for any quasicoherent sheaf of $\mathcal{O}_{T}$-modules $\mathcal{F}$ we have

$$
R^{2} \pi_{*}\left(\mathcal{L}^{\otimes r} \otimes \mathcal{F}\right)=0,
$$

therefore the formation of $R^{1} \pi_{*}\left(\mathcal{L}^{\otimes r} \otimes \mathcal{F}\right)$ commutes with base change on $T$ (see G-EGA, 7.3.1). 
Let $U$ be an affine scheme and $U \rightarrow \mathcal{M}$ a surjective étale morphism of finite type. Denote by $\mathcal{C}_{U}$ the pullback of $\mathcal{C}$ to $U$. Let $D \subset \mathcal{C}_{U}$ be the subcurve consisting of components of fibers mapping to a point in $U$. Note that, since $T$ is noetherian, the number of topological types occurring in the fibers of $D \rightarrow T$ is finite. Moreover, it follows from Lemma 9.2.1 and statement (3) in Lemma 9.2.2 that the inverse images of the nonstable trees of $\mathcal{C}$ in $\mathcal{C}_{U}$ are disjoint unions of trees of rational curves.

To check that $R^{1} \pi_{*}\left(\mathcal{L}^{\otimes r} \otimes \mathcal{F}\right)=0$, it is enough to check the case where $\mathcal{F}$ is the structure sheaf of a geometric point of $T$. This is a consequence of the following elementary lemma, whose proof is left to the reader:

Lemma 9.3.2. Let $E$ be a quasiprojective nodal curve, and let $D \subset E$ be the maximal proper subcurve. Then there exists a number $N$, depending only on the topological type of $D$, such that the following holds: Let $\mathcal{L}$ be an invertible sheaf satisfying the following properties:

(1) for any component $D^{\prime} \subset D$, the degree of $\mathcal{L}$ on $D^{\prime}$ is either $>N$ or 0 , and

(2) the union of the components $D^{\prime} \subset D$ such that $\operatorname{deg}_{D^{\prime}} \mathcal{L}=0$ is a disjoint union of trees of rational curves.

Then

- $H^{1}(E, \mathcal{L})=0$, and

- the algebra $\bigoplus H^{0}\left(E, \mathcal{L}^{\otimes k}\right)$ is generated in degree 1.

- Let $E^{\prime}=\operatorname{Proj} \oplus H^{0}\left(E, \mathcal{L}^{\otimes k}\right)$. Then $E^{\prime}$ is the seminormal curve obtained by contracting the components of $D$ on which $\mathcal{L}$ has degree 0.

This concludes the proof of part (1) of the Claim. Also, once we prove part (2), then Lemma 9.3.2 implies part (3).

For part (2), note that since $R^{1} \pi_{*}\left(\mathcal{L}^{\otimes r} \otimes \mathcal{F}\right)=0$, the formation of $\pi_{*} \mathcal{L}^{\otimes r}$ commutes with base change on $T$. This means that for any quasicoherent sheaf of $\mathcal{O}_{T}$-modules $\mathcal{F}$ we have the equality $\pi_{*}\left(\mathcal{L}^{\otimes r} \otimes \mathcal{F}\right)=\pi_{*} \mathcal{L}^{\otimes r} \otimes \mathcal{F}$. But the functor sending $\mathcal{F}$ to $\pi_{*}\left(\mathcal{L}^{\otimes r} \otimes \mathcal{F}\right)$ is evidently left exact, which means that the sheaf is flat. This concludes the proof of the Claim.

Define $\mathcal{C}^{\prime}:=\operatorname{Proj}_{\mathcal{M}} \mathcal{A} \rightarrow \mathcal{M}$.

Step 4: verification that $\mathcal{C}^{\prime} \rightarrow \mathcal{M}$ is a tWisted stable pointed Map.

Since $\mathcal{C}^{\prime} \rightarrow \mathcal{M}$ is projective, it is representable. Since $\pi_{*} \mathcal{L}^{\otimes k}$ is flat over $T$ for all $k>0$, we have that $\mathcal{C}^{\prime} \rightarrow T$ is flat.

Let $\Sigma_{i}^{\mathcal{C}}$ be one of the markings. We claim that the composition $\Sigma_{i}^{\mathcal{C}} \rightarrow \mathcal{C} \rightarrow \mathcal{C}^{\prime}$ is an embedding, and we define $\Sigma_{i}^{\mathcal{C}^{\prime}}$ to be its image in $\mathcal{C}^{\prime}$. This, as well as the fact that $\mathcal{C}^{\prime} \rightarrow \mathcal{M}$ satisfies statement (1) of the proposition, can be checked on geometric fibers.

We claim that statement (2) can also be checked on the level of geometric fibers. First, the local criterion of flatness implies that a proper morphism $C_{1} \rightarrow C_{2}$ of flat $T$-schemes which is an isomorphism on the geometric fibers of $T$ is an isomorphism. Also, the formation of coarse moduli spaces of tame stacks commutes with arbitrary base change (see Lemma 2.3.3). Since the moduli space of a family of twisted nodal curves is flat, the claim follows.

So we assume that $T$ is the spectrum of an algebraically closed field.

The formation of the algebra $\mathcal{A}$ commutes with base change along étale covers $U \rightarrow \mathcal{M}$. It follows from Lemma 9.3.2 that over $U$, the curve $\mathcal{C}_{U}^{\prime}=\operatorname{Proj}_{U} \mathcal{A}_{U}$ is the contraction of the nonstable trees in $\mathcal{C}_{U}$. Using descent and the uniqueness of the 
contraction, we have that $\mathcal{C} \rightarrow \mathcal{C}^{\prime} \rightarrow \mathcal{M}$ satisfies statement (1) in the proposition. Denote by $\overline{C^{\prime}}$ the moduli space of $\mathcal{C}^{\prime}$. The morphism $\overline{C^{\prime}} \rightarrow C^{\prime}$ is a bijection on geometric points and is an isomorphism away from the special points, and since $C^{\prime}$ is seminormal, the morphism is an isomorphism.

\section{Appendix A. GrothendieCK's existence theOrem For tAme StACKs}

A.1. Statement of the existence theorem. Consider a proper tame stack $\mathcal{X}$ of finite type over a noetherian complete local ring $A$ with maximal ideal $\mathfrak{m}$. Then $\mathcal{X}$ has a moduli space $\mathbf{X}$, which is a proper algebraic space over $A$.

For each nonnegative integer $n$ set $\mathcal{X}_{n}=\mathcal{X} \times \times_{\operatorname{Spec} A} \operatorname{Spec} A / \mathfrak{m}^{n+1}$. We define the category of formal coherent sheaves on $\mathcal{X}$ in a rather simple-minded way. A formal coherent sheaf $\widehat{\mathcal{F}}$ on $\mathcal{X}$ is a collection $\left(\mathcal{F}_{n}\right)_{n \geq 0}$ of coherent sheaves on $\mathcal{O}_{\mathcal{X}_{n}}$, together with isomorphisms $\left.\mathcal{F}_{n+1}\right|_{\mathcal{X}_{n}} \simeq \mathcal{F}_{n}$ of sheaves over $\mathcal{O}_{X_{n}}$. A homomorphism $\widehat{\mathcal{F}} \rightarrow \widehat{\mathcal{G}}$ of formal coherent sheaves is a compatible sequence of morphisms $\mathcal{F}_{n} \rightarrow \mathcal{G}_{n}$, in the obvious way. We shall denote the category of formal coherent sheaves on $\mathcal{X}$ by $\mathrm{F} \operatorname{Coh}(\mathcal{X})$. From this description it is not even clear that $\mathrm{F} \operatorname{Coh}(\mathcal{X})$ is abelian.

There is an obvious functor from the category $\operatorname{Coh}(\mathcal{X})$ of coherent sheaves on $\mathcal{X}$ to $\mathrm{F} \operatorname{Coh}(\mathcal{X})$, sending a coherent sheaf $\mathcal{F}$ to the compatible system of restrictions $\left.\mathcal{F}\right|_{\mathcal{X}_{n}}$.

Theorem A.1.1. If $\mathcal{X}$ is proper and tame over $\operatorname{Spec} A$, the functor $\operatorname{Coh}(\mathcal{X}) \rightarrow$ $\mathrm{F} \operatorname{Coh}(\mathcal{X})$ is an equivalence of categories.

This is of course well known if $\mathcal{X}$ is an algebraic space (see $[\mathrm{Kn}, \mathrm{V}, 6.3$ ). The proof for stacks is not too different from the one in $[\mathrm{Kn}]$.

A.2. Restatement in terms of $\widehat{\mathcal{O}}_{\mathcal{X}}$-modules. For the proof, we will need a more manageable description of the category of formal coherent sheaves. Consider the étale site $\mathcal{X}_{\text {ét }}$ of $\mathcal{X}$, where the objects are the étale morphisms $U \rightarrow \mathcal{X}$, where $U$ is a scheme, the arrows are maps over $\mathcal{X}$, and coverings are defined as usual ( $\mathrm{L}-\mathrm{MB})$. On this site we have two sheaves of rings; the usual structure sheaf, which we denote by $\mathcal{O}_{\mathcal{X}}$, which sends each étale map $U \rightarrow \mathcal{X}$ to $\mathcal{O}(U)$, and the completed structure sheaf $\widehat{\mathcal{O}}_{\mathcal{X}}$, which sends an étale map $U \rightarrow \mathcal{X}$ to the completion of $\mathcal{O}(U)$ with respect to the inverse image of the maximal ideal of $A$. We denote by $\widehat{\mathcal{X}}$ the ringed site $\mathcal{X}_{\text {ét }}$, equipped with the latter sheaf of rings $\widehat{\mathcal{O}}_{\mathcal{X}}$.

Notice that in the case that $\mathcal{X}$ is a scheme, the site $\widehat{\mathcal{X}}$ is not the étale site of the corresponding formal scheme; however, since the structure sheaf of the formal scheme is supported on the closed fiber, the two categories of sheaves of modules on $\widehat{\mathcal{X}}$ and on the étale site of the formal scheme are canonically equivalent, so this does not really make a difference.

Consider a sheaf of $\widehat{\mathcal{O}}_{\mathcal{X}}$-modules $\mathcal{F}$. Let $U \rightarrow \mathcal{X}$ be an étale map, where $U$ is a scheme, and let $\widehat{\mathcal{O}}_{U}$ be the restriction of $\widehat{\mathcal{O}}_{\mathcal{X}}$ to the Zariski site of $U$. The sheaf $\widehat{\mathcal{O}}_{\mathcal{X}}$ is actually supported on the fiber $U_{0}=U \times_{\operatorname{Spec} A} \operatorname{Spec} A / \mathfrak{m}$, so the restriction $\left.\mathcal{F}\right|_{U}$ of $\mathcal{F}$ to $U$ defines a sheaf on the formal completion $\widehat{U}$ of the scheme $U$ along $U_{0}$. We say that $\mathcal{F}$ is a coherent sheaf on $\widehat{\mathcal{X}}$ when $\left.\mathcal{F}\right|_{U}$ is a coherent sheaf on the formal scheme $\widehat{U}$ for all étale maps $U \rightarrow \mathcal{X}$. The category of coherent sheaves of modules over $\widehat{\mathcal{X}}$ will be denoted by $\operatorname{Coh}(\widehat{\mathcal{X}})$; it is clearly an abelian category.

There is an obvious functor $\operatorname{Coh}(\widehat{\mathcal{X}}) \rightarrow \mathrm{F} \operatorname{Coh}(\mathcal{X})$ that sends a coherent sheaf of modules $\mathcal{F}$ over $\widehat{\mathcal{X}}$ to the object $\widehat{\mathcal{F}} \stackrel{\text { def }}{=}\left(\mathcal{F} \otimes_{\widehat{\mathcal{O}}_{\mathcal{X}}} \mathcal{O}_{\mathcal{X}_{n}}\right)_{n \geq 0}$ of $\mathrm{F} \operatorname{Coh}(\mathcal{X})$. This functor 
is easily checked to be an equivalence of categories; the inverse is obtained by taking inverse limits in the usual fashion, i.e., one can send an object $\left(\mathcal{F}_{i}\right)$ of $\mathrm{F} \operatorname{Coh}(\mathcal{X})$ into $\lim \mathcal{F}_{i}$, and this is a coherent sheaf of modules over $\widehat{\mathcal{X}}$.

Now, the functor $\operatorname{Coh}(\mathcal{X}) \rightarrow \mathrm{F} \operatorname{Coh}(\mathcal{X})$ above corresponds to the functor $\operatorname{Coh}(\mathcal{X})$ $\rightarrow \operatorname{Coh}(\widehat{\mathcal{X}})$ which sends a coherent sheaf $\mathcal{F}$ to $\widehat{\mathcal{F}} \stackrel{\text { def }}{=} \mathcal{F}$. This induces a natural morphism $\mathrm{H}^{i}(\mathcal{X}, \mathcal{F}) \rightarrow \mathrm{H}^{i}(\widehat{\mathcal{X}}, \widehat{\mathcal{F}})$ from the cohomology of $\mathcal{F}$ on $\mathcal{X}$ to the cohomology of the completion of $\mathcal{F}$ on $\widehat{\mathcal{X}}$. The first important step of the proof of the theorem consists of proving the following form of Zariski's theorem on formal functions.

A.3. The theorem on formal functions. In what follows we will use repeatedly the well-known fact that the cohomology of a coherent sheaf, considered as a sheaf on the small étale site of a scheme, is the same as the cohomology of the same sheaf on the Zariski site.

Theorem A.3.1. If $\mathcal{X}$ is proper over $\operatorname{Spec} A$, the morphism $\mathrm{H}^{i}(\mathcal{X}, \mathcal{F}) \rightarrow \mathrm{H}^{i}(\widehat{\mathcal{X}}, \widehat{\mathcal{F}})$ is an isomorphism.

Lemma A.3.2. Let $\left(\mathcal{F}_{i}\right)_{i \geq 0}$ be a formal sheaf on $\mathcal{X}$, and set $\widehat{\mathcal{F}}=\lim \mathcal{F}_{i}$. Then the natural map $\mathrm{H}^{n}(\mathcal{X}, \widehat{\mathcal{F}}) \rightarrow \lim ^{n} \mathrm{H}^{n}\left(\mathcal{X}, \mathcal{F}_{i}\right)$ is an isomorphism.

Proof. This is very much the proof of the corresponding fact in [ $\mathrm{Kn}], \mathrm{V}, 2.19$, except that it is, hopefully, correct. Let $U \rightarrow \mathcal{X}$ be a surjective étale map, where $U$ is an affine scheme. Then all the fiber products $U^{n}=U \times \times_{\mathcal{X}} U \times_{\mathcal{X}} \cdots \times_{\mathcal{X}} U \times \times_{\mathcal{X}} U$ are affine, because $\mathcal{X}$ is separated. The usual spectral sequence

$$
\check{\mathrm{H}}^{p}\left(U / \mathcal{X}, \mathcal{H}^{q}\left(\mathcal{F}_{i}\right)\right) \Longrightarrow \mathrm{H}^{p+q}\left(\mathcal{X}, \mathcal{F}_{i}\right)
$$

relating Čech cohomology and usual cohomology degenerates, because the cohomology of $\mathcal{F}_{i}$ over each $U^{n}$ degenerates, so cohomology is equal to Čech cohomology over the covering $U \rightarrow \mathcal{X}$. We claim that the same is true for the projective limit

$$
\widehat{\mathcal{F}} \stackrel{\text { def }}{=} \lim _{\longleftarrow} \mathcal{F}_{i} .
$$

For this it is enough to show that the cohomology of $\widehat{\mathcal{F}}$ on each $U^{n}$ is zero in positive degree. This is the content of the lemma that follows.

Lemma A.3.3. The cohomology of the limit of a strict projective system of coherent sheaves on the small étale site of an affine noetherian scheme $X$ is zero in positive degree.

Proof. Let $X$ be the affine scheme, $\left(\mathcal{F}_{i}\right)$ the projective system, and $\widehat{\mathcal{F}}$ the limit. According to a theorem of M. Artin (see [Mi], Theorem 2.17), the Cech cohomology of $\widehat{\mathcal{F}}$ on the small étale site $X_{\text {ét }}$ equals its cohomology; so it is enough to show that, given an étale map $U \rightarrow X$ of finite type, with $U$ affine, the Cech cohomology $\check{\mathrm{H}}^{\bullet}(U / X, \widehat{\mathcal{F}})$ vanishes in positive degree. Let us denote by $K_{\bullet}(\mathcal{G})$ the augmented Čech complex

$$
\Gamma(X, \mathcal{G}) \longrightarrow \Gamma(U, \mathcal{G}) \longrightarrow \Gamma\left(U^{2}, \mathcal{G}\right) \longrightarrow \cdots
$$

of a sheaf $\mathcal{G}$.

By the strictness assumption, each of the maps $\mathcal{F}_{i+1} \rightarrow \mathcal{F}_{i}$ is surjective, so $\mathcal{F}_{i+1}\left(U^{n}\right) \rightarrow \mathcal{F}_{i}\left(U^{n}\right)$ is surjective for each $n$, because $U^{n}$ is affine and $\mathcal{F}_{i}$ is coherent. 
Thus the projective system

$$
\cdots \rightarrow K_{\bullet}\left(\mathcal{F}_{i+1}\right) \rightarrow K_{\bullet}\left(\mathcal{F}_{i}\right) \rightarrow \cdots
$$

is a projective system of acyclic complexes satisfying the Mittag-Leffler condition in each degree, and therefore its projective limit

$$
\varliminf_{\bullet} K_{\bullet}\left(\mathcal{F}_{i}\right)=K_{\bullet}(\widehat{\mathcal{F}})
$$

is acyclic.

To conclude the proof of Lemma A.3.2, it is enough to show that the map

$$
\check{\mathrm{H}}^{n}(U / \mathcal{X}, \widehat{\mathcal{F}}) \rightarrow \lim _{\longleftarrow} \check{\mathrm{H}}^{n}\left(U / \mathcal{X}, \mathcal{F}_{i}\right)
$$

is an isomorphism. The Čech complexes $\check{\mathrm{C}}^{\bullet}\left(U / \mathcal{X}, \mathcal{F}_{i}\right)$ form a projective system of complexes which satisfy a Mittag-Leffler condition in each degree. Furthermore the cohomology groups $\check{\mathrm{H}}^{n}\left(U / \mathcal{X}, \mathcal{F}_{i}\right)$ are artinian modules over $A$, so the corresponding projective systems also satisfy a Mittag-Leffler condition. The statement follows from [G-EGA], 0, 13.2.3.

Consider the moduli space $\pi: \mathcal{X} \rightarrow \mathbf{X}$.

Lemma A.3.4. The natural map $\left(\pi_{*} \mathcal{F}\right)_{r} \rightarrow \pi_{*}\left(\mathcal{F}_{r}\right)$ is an isomorphism.

Proof. This statement is local in the étale topology, so we may assume that $\mathcal{X}=$ $[Y / \Gamma], Y=\operatorname{Spec} R, \mathbf{X}=\operatorname{Spec} R^{\Gamma}$. The sheaf $\mathcal{F}$ is associated with the $\Gamma$-equivariant $R$-module $M$, and $\pi_{*} \mathcal{F}$ corresponds with the $R^{\Gamma}$-module $M^{\Gamma}$. Then the statement corresponds to the fact that the natural map $M^{\gamma} / \mathfrak{m}^{r+1} M^{\Gamma} \rightarrow\left(M / \mathfrak{m}^{r+1} M\right)^{\Gamma}$ is an isomorphism. The surjectivity follows immediately from the fact that any invariant in the right-hand side lifts to an invariant in $M$. For the injectivity, take an element $m$ in $M^{\Gamma}$ which goes to 0 in $\left(M / \mathfrak{m}^{r+1} M\right)^{\Gamma}$; then we can write $m=\sum_{i} a_{i} m_{i}$, where $a_{i} \in \mathfrak{m}^{r+1}$ and $m_{i} \in M$. By averaging, we may assume that the $m_{i}$ are invariant, so that $m \in \mathfrak{m}^{r+1} M^{\Gamma}$.

To complete the proof of the theorem of formal function A.3.1 notice that from the theorem on formal functions for algebraic spaces $(\mathrm{Kn}, \mathrm{V}, 3.1)$, we conclude that

$$
\mathrm{H}^{n}(\mathcal{X}, \mathcal{F})=\mathrm{H}^{n}\left(\mathbf{X}, \pi_{*} \mathcal{F}\right)=\varliminf_{\lim } \mathrm{H}^{n}\left(\mathbf{X}, \pi_{*} \mathcal{F}_{r}\right)=\lim _{\longleftarrow} \mathrm{H}^{n}\left(\mathcal{X}, \mathcal{F}_{r}\right) .
$$

\section{A.4. Algebraization of extensions, kernels, and cokernels.}

Lemma A.4.1. Let $\mathcal{F}$ and $\mathcal{G}$ be two coherent sheaves on $\mathcal{X}$. The natural map

$$
\mathcal{E} x t_{\mathcal{O}_{\mathcal{X}}}^{n}(\mathcal{F}, \mathcal{G})^{\wedge} \rightarrow \mathcal{E} x t_{\widehat{\mathcal{O}}_{\mathcal{X}}}^{n}(\widehat{\mathcal{F}}, \widehat{\mathcal{G}})
$$

is an isomorphism for all $n$.

Proof. This is a local statement in the étale topology, so it follows from the case that $\mathcal{X}$ is a scheme.

Lemma A.4.2. Let $\mathcal{F}$ and $\mathcal{G}$ be two coherent sheaves on $\mathcal{X}$. The natural map

$$
\operatorname{Ext}_{\mathcal{O}_{\mathcal{X}}}^{n}(\mathcal{F}, \mathcal{G}) \rightarrow \operatorname{Ext}_{\hat{\mathcal{O}}_{\mathcal{X}}}^{n}(\widehat{\mathcal{F}}, \widehat{\mathcal{G}})
$$

is an isomorphism for all $n$. 
Proof. This follows from the theorem on formal functions and Lemma A.4.1 above, by considering the local global spectral sequences for $\mathcal{E} x t_{\mathcal{O}_{\mathcal{X}}}^{n}(\mathcal{F}, \mathcal{G})$ and $\mathcal{E} x t_{\widehat{\mathcal{O}}_{\mathcal{X}}}^{n}(\widehat{\mathcal{F}}, \widehat{\mathcal{G}})$.

This implies that the functor $\operatorname{Coh}(\mathcal{X}) \rightarrow \operatorname{Coh}(\widehat{\mathcal{X}})$ is fully faithful. We have to prove that all formal coherent sheaves on $\widehat{\mathcal{X}}$ are algebraizable, that is, it is isomorphic to the completion of a coherent sheaf on $\mathcal{X}$.

Lemma A.4.3. The kernel and the cokernel of a morphism of algebraizable formal sheaves are algebraizable.

Proof. Let $\mathcal{F}$ and $\mathcal{G}$ be coherent $\mathcal{O}_{\mathcal{X}}$-modules, and $\widehat{\phi}: \widehat{\mathcal{F}} \rightarrow \widehat{\mathcal{G}}$ a morphism of $\widehat{\mathcal{O}}_{\mathcal{X}}$ modules. By the previous result, $\widehat{\phi}$ comes from a morphism of $\mathcal{O}_{\mathcal{X}}$-modules $\phi: \mathcal{F} \rightarrow$ $\mathcal{G}$. Since completion is an exact functor, the kernel and the cokernel of $\widehat{\phi}$ are the completion of the kernel and cokernel of $\phi$.

Lemma A.4.4. Any extension of algebraizable formal sheaves is algebraizable.

Proof. If $\mathcal{F}$ and $\mathcal{G}$ are coherent $\mathcal{O}_{\mathcal{X}}$-modules, the natural map

$$
\operatorname{Ext}_{\mathcal{O}_{\mathcal{X}}}^{1}(\mathcal{F}, \mathcal{G}) \rightarrow \operatorname{Ext}_{\widehat{\mathcal{O}}_{\mathcal{X}}}^{1}(\widehat{\mathcal{F}}, \widehat{\mathcal{G}})
$$

is an isomorphism, by Lemma A.4.2

A.5. Proof of the existence theorem. By noetherian induction, in order to prove the existence theorem we may assume that every coherent sheaf on $\mathcal{X}$ is algebraizable when it is zero on an open nonempty substack of $\mathcal{X}$. Let $\rho: Y \rightarrow \mathcal{X}$ be a surjective finite generically étale map, where $Y$ is a scheme; this exists by [L-MB], 16.6. If $\widehat{Y}$ is the completion of $Y$, then the diagram of ringed Grothendieck topologies

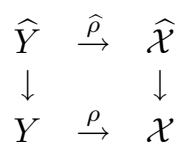

is cartesian. From this we deduce the following lemma.

Lemma A.5.1. Let $\mathcal{F}$ be a coherent sheaf on $Y$. Then the natural map $\widehat{\rho_{*} \mathcal{F}} \rightarrow \widehat{\rho}_{*} \widehat{\mathcal{F}}$ is an isomorphism.

Lemma A.5.2. Let $\overline{\mathcal{F}}$ be a coherent sheaf on $\widehat{Y}$. Then the sheaf $\widehat{\rho}_{*} \overline{\mathcal{F}}$ is a coherent sheaf of $\mathcal{O}_{\hat{\mathcal{X}}}$-modules.

Proof. By restricting to an étale map $U \rightarrow \mathcal{X}$, where $U$ is an affine scheme considered with the Zariski topology, this becomes obvious.

From these two lemmas we see that a formal sheaf on $\widehat{\mathcal{X}}$ of the form $\widehat{\rho}_{*} \overline{\mathcal{F}}$ is algebraizable.

Now, we form the morphism $\sigma: Y \times \mathcal{X} Y \rightarrow \mathcal{X}$. Let $\overline{\mathcal{F}}$ be a formal coherent sheaf on $\mathcal{X}$; there are two natural maps $\psi_{1}$ and $\psi_{2}$ from $\widehat{\rho}_{*} \widehat{\rho}^{*} \overline{\mathcal{F}}$ to $\widehat{\sigma}_{*} \widehat{\sigma}^{*} \overline{\mathcal{F}}$ induced by the two projections $Y \times \mathcal{X} Y \rightarrow Y$; let $\overline{\mathcal{K}}$ be the kernel of the difference $\psi_{1}-\psi_{2}$. The adjunction map $\overline{\mathcal{F}} \rightarrow \widehat{\rho}_{*} \widehat{\rho}^{*} \overline{\mathcal{F}}$ factors through $\overline{\mathcal{K}}$, and, by flat descent, the induced map $\alpha: \overline{\mathcal{F}} \rightarrow \overline{\mathcal{K}}$ is an isomorphism over the open dense substack of $\mathcal{X}$ where the map $\rho$ is flat. By Lemma A.4.3 $\mathcal{K}$ is algebraizable; also, the kernel and the cokernel of the map $\alpha$ are algebraizable, by the induction hypothesis. So the image of $\alpha$ is algebraizable, and therefore by the same lemma $\overline{\mathcal{F}}$ is algebraizable. 


\section{REFERENCES}

$[\aleph-\mathrm{C}-\mathrm{V}]$ D. Abramovich, A. Corti and A. Vistoli, Twisted bundles and admissible covers, preprint, 2001, math.AG/0106211.

$[\aleph-G-V] \quad$ D. Abramovich, T. Graber and A. Vistoli, Algebraic orbifold quantum products, in preparation.

[§-J] D. Abramovich and T. Jarvis, Moduli of twisted spin curves, preprint, math. AG/0104154.

[א-O] D. Abramovich and F. Oort, Stable maps and Hurwitz schemes in mixed characteristic. In: Advances in Algebraic Geometry Motivated by Physics, Emma Previato (ed.), AMS, 2001. MR 2001h:14009

[§-V1] D. Abramovich and A. Vistoli, Complete moduli for families over semistable curves, preprint, math.AG/9811059.

[§-V2] D. Abramovich and A. Vistoli, Complete moduli for fibered surfaces. In Recent Progress in Intersection Theory, G. Ellingsrud, W. Fulton, A. Vistoli (eds.), Birkhäuser, 2000.

[Ar] M. Artin, Versal deformations and algebraic stacks, Invent. Math. 27 (1974), 165-189. MR 53:2945

[B-M] Kai Behrend and Yuri I. Manin, Stacks of stable maps and Gromov-Witten invariants, Duke Math. J. 85 (1996), no. 1, 1-60. MR 98i:14014

[B-G-I] P. Berthelot, A. Grothendieck and L. Illusie, Théorie des intersections et théorème de Riemann-Roch, Séminaire de Géométrie Algébrique du Bois-Marie 1966-1967 (SGA 6). Dirigé par P. Berthelot, A. Grothendieck et L. Illusie. Avec la collaboration de D. Ferrand, J. P. Jouanolou, O. Jussila, S. Kleiman, M. Raynaud et J. P. Serre. Lecture Notes in Mathematics, Vol. 225, Springer, Berlin, 1971. MR 50:7133

[C-R] W. Chen and Y. Ruan, Orbifold Gromov-Witten theory, preprint, math.AG/0103156.

[D-M] P. Deligne and D. Mumford, The irreducibility of the space of curves of given genus, Inst. Hautes Études Sci. Publ. Math. No. 36 (1969), 75-109. MR 41:6850

[E-H-K-V] D. Edidin, B. Hassett, A. Kresch and A. Vistoli, Brauer groups and quotient stacks, American Journal of Mathematics, to appear, math.AG/9905049.

[E-G] D. Edidin and W. Graham, Equivariant intersection theory, Invent. Math. 131 (1998), no. 3, 595-634. MR 99j:14003a

[F-G] B. Fantechi and L. Göttsche, Orbifold cohomology for global quotients, preprint, math. AG/0104207.

[F-P] William Fulton and Rahul Pandharipande, Notes on stable maps and quantum cohomology. in Algebraic geometry - Santa Cruz 1995, Proc. Sympos. Pure Math., Part 2, Amer. Math. Soc., Providence, RI, 1997, 45-96. MR 98m:14025

[G-EGA] A. Grothendieck, Éléments de géométrie algébrique. III. Étude cohomologique des faisceaux cohérents. I, Inst. Hautes Études Sci. Publ. Math. No. 11 (1961), 167 pp.; II, Inst. Hautes Études Sci. Publ. Math. No. 17 (1963), 91 pp. MR 36:177c MR 29:1210

[G-FGA] A. Grothendieck, Fondements de la géométrie algébrique. Extraits du Sém. Bourbaki, 1957 - 1962. Secrétariat Math., Paris, 1962. MR 26:3566

[G-SGA1] A. Grothendieck, Revêtements étales et groupe fondamental, Séminaire de Géométrie Algébrique du Bois Marie 1960-1961 (SGA 1). Dirigé par A. Grothendieck. Augmenté de deux exposés de M. Raynaud. Lecture Notes in Mathematics, Vol. 224, Springer, Berlin, 1971. MR 50:7129

[Il] L. Illusie, Complexe cotangent et déformations. I, II, Lecture Notes in Mathematics, Vol. 239, Springer, Berlin, 1971 \& Lecture Notes in Mathematics, Vol. 283, Springer, Berlin, 1972. MR 58:10886a; MR 58:10886b

[dJ-O] A. J. de Jong and F. Oort, On extending families of curves, J. Alg. Geom. 6 (1997), no. 3, 545-562. MR 99f:14028

[K-M] S. Keel and S. Mori, Quotients by groupoids, Ann. of Math. (2) 145 (1997), no. 1, 193-213. MR 97m:14014

[K-S] G. M. Kelly and R. Street, Review of the elements of 2-categories. In Category Seminar (Proc. Sem., Sydney, 1972/1973), Lecture Notes in Math., Vol. 420, Springer, Berlin, 1974. MR 50:10010

[Kn] D. Knutson, Algebraic spaces, Lecture Notes in Math., Vol. 203, Springer, Berlin, 1971. MR 46:1791 
[Ko] M. Kontsevich, Enumeration of rational curves via torus actions. In The moduli space of curves (Texel Island, 1994), 335-368, Progr. Math., 129, Birkhäuser, Boston, Boston, MA, 1995. MR 97d:14077

[Kr] A. Kresch, Cycle groups for Artin stacks, Inventiones Mathematicae 138 (1999), 495536. MR 2001a:14003

[La] G. La Nave, Stable reduction for elliptic surfaces with sections, Ph.D. Thesis, Brandeis University, 2000.

[L-MB] G. Laumon and L. Moret-Bailly, Champs Algébriques, Ergebnisse der Mathematik und ihrer Grenzgebiete 39, Springer-Verlag, 2000. MR 2001f:14006

[Ma] H. Matsumura, Commutative ring theory, Translated from the Japanese by M. Reid, Second edition, Cambridge Univ. Press, Cambridge, 1989. MR 90i:13001

[Mi] J. S. Milne, Étale cohomology, Princeton Univ. Press, Princeton, N.J., 1980. MR 81j:14002

[Mo] S. Mochizuki, Extending families of curves over log regular schemes, J. Reine Angew. Math. 511 (1999), 43-71. MR 2000f:14041

[Vi] A. Vistoli, Intersection theory on algebraic stacks and on their moduli spaces, Invent. Math. 97 (1989), no. 3, 613-670. MR 90k:14004

[We] S. Wewers, Construction of Hurwitz spaces, Institut für Experimentelle Mathematik preprint No. 21 (1998).

Department of Mathematics, Boston University, 111 Cummington Street, Boston, Massachusetts 02215

E-mail address: abrmovic@math.bu.edu

Dipartimento di Matematica, Università di Bologna, Piazza di Porta San Donato 5, 40127 Bologna, ITALY

E-mail address: vistoli@dm.unibo.it 